\title{
On a Polyanalytic Approach to Noncommutative de Branges-Rovnyak Spaces and Schur Analysis
}

\author{
Daniel Alpay, Fabrizio Colombo, Kamal Diki and Irene Sabadini®
}

\begin{abstract}
In this paper we begin the study of Schur analysis and of de Branges-Rovnyak spaces in the framework of Fueter hyperholomorphic functions. The difference with other approaches is that we consider the class of functions spanned by Appell-like polynomials. This approach is very efficient from various points of view, for example in operator theory, and allows us to make connections with the recently developed theory of slice polyanalytic functions. We tackle a number of problems: we describe a Hardy space, Schur multipliers and related results. We also discuss Blaschke functions, Herglotz multipliers and their associated kernels and Hilbert spaces. Finally, we consider the counterpart of the half-space case, and the corresponding Hardy space, Schur multipliers and Carathéodory multipliers.
\end{abstract}

Mathematics Subject Classification. 47B32, 47S05, 30G35.

Keywords. Quaternions, Fueter hyperholomorphic functions, Slice polyanalytic functions, Appell polynomials, Schur analysis, Hardy space.

\section{Introduction}

Two important function theories that allow to extend complex analysis and operator theory results to higher dimensions are the so-called monogenic and slice monogenic functions with values in a Clifford algebra. In the case of quaternions these two theories are known as Fueter hyperholomorphic and slice regular or slice hyperholomorphic functions, respectively, see $[32,38,39$, $61,63]$. For the necessary preliminaries on quaternions, we refer the reader to Sect. 2. An interesting problem is to investigate the possible relations and intersections between these two different theories. We note that it is always possible to construct Fueter hyperholomorphic functions starting from slice regular ones using different techniques such as the Fueter mapping theorem $[36,37]$, or using the Radon and dual Radon transforms, see [35]. But in general, the slice monogenicity does not imply, nor is implied by monogenicity. 
However, in $[14,15]$ the authors extended the notion of slice regular functions to higher order by considering the so-called slice polyanalytic functions. These functions can be considered from three different points of view. The first approach consists of viewing the space of quaternions $\mathbb{H}$ as union of complex planes and to see these functions as a subclass of null solutions of the $n$-th power of the Cauchy-Riemann operator with respect to each complex plane. The second approach is based on the so-called poly-decomposition which allows us to consider such functions as sums of the form

$$
\sum_{k=0}^{n-1} \bar{x}^{k} f_{k}(x), \quad x \in \mathbb{H}
$$

with all the components $f_{k}$ which are slice regular functions and $n$ is the order of poly-analyticity. The third approach consists in considering slice polyanalytic functions as subclass of the null solutions of the $n$-th power of a global operator with non-constant coefficients, see [13]. The study in this paper is in the quaternionic context and it is based on some polynomials $\left(P_{n}(x)\right)_{n \geq 0}$ where

$$
P_{n}(x)=\sum_{j=0}^{n} L_{j, n} \bar{x}^{j} x^{n-j}, \quad n \geq 0,
$$

that are at the same time Fueter hyperholomorphic and slice polyanalytic functions of order $n+1$, for suitable real coefficients $L_{j, n}$ (see [34,54] and Sect. 2). These polynomials are very special since they belong to the intersection of two different non-commutative function theories, namely the classical Fueter theory and the slice polyanalytic theory, moreover they have nice properties with respect to multiplication and derivation. Another important feature, see Theorem 3.10 in [12], is that any Fueter hyperholomorphic function $f$ of axial type admits a power series expansion in terms of the polynomials $P_{n}$ of the form

$$
f(x)=\sum_{n=0}^{\infty} P_{n}(x) u_{n}, \quad u_{n} \in \mathbb{H} .
$$

This fact allows to embed the space of Fueter hyperholomorphic functions of axial type, denoted by $\mathcal{A R}$, into a space consisting of series of slice polynalytic functions that we denote here by

$$
\mathcal{S P} \mathcal{P}_{\infty}:=\mathcal{S} \mathcal{P}_{1}+\mathcal{S} \mathcal{P}_{2}+\cdots+\mathcal{S} \mathcal{P}_{n+1}+\cdots
$$

where $\mathcal{S} \mathcal{P}_{n}$ denotes the set of slice polyanalytic functions of order $n$. More precisely we consider the subspaces of slice polyanalytic functions associated with the polynomials $\left(P_{n}\right)_{n \geq 0}$ defined by

$$
\mathcal{P}_{n}:=\left\{P_{n}(x) \lambda, \lambda \in \mathbb{H}\right\}
$$

and

$$
\mathcal{P}_{\infty}:=\bigoplus_{n=0}^{\infty} \mathcal{P}_{n}
$$


Then, since the $P_{n}$ are the unique hyperholomorphic extensions of axial type of the real valued functions $\left(3 x_{0}\right)^{n}$, it is possible to show that the space of hyperholomorphic functions of axial type $\mathcal{A R}$ corresponds to the space $\mathcal{P}_{\infty}$, i.e.

$$
\mathcal{A R}=\mathcal{P}_{\infty}
$$

The previous subspaces of slice polyanalytic functions $\mathcal{P}_{n}$ were considered before from a different point of view and using a different terminology, namely they were called spaces of homogeneous special monogenic polynomials of degree $n$, see for example Lemma 1 in [3]. Using these ideas and identifications we show that it is always possible to embed this interesting subclass of special monogenic functions in a more general framework of slice polyanalytic functions. We use techniques from slice polyanalytic function theory to prove results on such special monogenic functions. In particular, in Proposition 2.18 we prove a Representation Formula in the monogenic setting using a slice polyanalytic approach.

Furthermore, we note that these slice polyanalytic (and Fueter hyperholomorphic) polynomials $\left(P_{n}\right)_{n \geq 0}$ are just a particular case of a more general interesting construction which makes use of the classical CauchyKovalevskaya extension theorem as we explain here. Consider an entire real analytic and quaternionic-valued function $h$ of the real variables $x_{1}, x_{2}, x_{3}$. The Cauchy-Kovalevskaya theorem guarantees the existence of a hyperholomorphic function $H$, its $C K$-extension. We have, with $h_{n}=h^{n}$ and $H_{n}=$ $H^{\odot n}$ (where $\odot$ denotes the Cauchy-Kovalevskaya product)

$$
C K\left(h_{n}\right) \odot C K\left(h_{m}\right)=C K\left(h_{m+n}\right)
$$

and so

$$
H_{n}=H_{1}^{n \odot} .
$$

We can see already here that obstructions occur; if we take quaternions $u$ and $v$, the $C K$-product $C K\left(h_{n} u\right) \odot C K\left(h_{m} v\right)$ will not be in general be equal to $C K\left(h_{n+m} u v\right)$ since $h_{m}$ and $u$ do not commute. As a consequence, the $C K$ product will not be, in general, translated into convolution of the coefficients of the expansions along the $H_{n}$. In spite of this, with this new variable $H_{1}$ it is possible to define a number of counterparts of the classical reproducing kernel Hilbert spaces, with reproducing kernel of the form

$$
K(x, y)=\sum_{n \in I} \frac{H_{n}(x) \overline{H_{n}(y)}}{\alpha_{n}}, \quad \alpha_{n}>0, \quad I \subset \mathbb{N}_{0},
$$

converging in some neighborhood of the origin in $\mathbb{R}^{4}$. We already mention at this point that the $C K$-product is not a law of composition for the Hardy space (defined below), and more generally, for series in the functions $H_{n}$.

The choice $I=\mathbb{N}_{0}$ and $\alpha_{n}=1$ for every $n \in \mathbb{N}_{0}$ corresponds to the underlying Hardy space, consisting of functions of the form

$$
f(x)=\sum_{n=0}^{\infty} H_{n}(x) f_{n}
$$


where $f_{0}, f_{1}, \ldots \in \mathbb{H}$ and satisfy $\sum_{n=0}^{\infty}\left|f_{n}\right|^{2}<\infty$. These functions are hyperholomorphic in

$$
\Omega=\left\{x \in \mathbb{R}^{4} ;\left|H_{1}(x)\right|<1\right\}
$$

since the radius of convergence of Cauchy-Kovalevskaya product satisfies $\rho\left(H_{n}\right) \leq\left(\rho\left(H_{1}\right)\right)^{n}$; see [23, Proposition 2.9, p. 131].

A corresponding Schur analysis would consist in particular of the following problems:

- Characterize the contractive multipliers of this Hardy space. The definition has to be adapted to the present situation, where we lack the convolution of the coefficients and the $C K$-product is not a law of composition.

- Study interpolation problems for these multipliers.

- Study the de Branges-Rovnyak spaces. These are families of Hilbert spaces of analytic functions, with reproducing kernels of various forms; see $[40,41,43,44,51,52,57,58]$. Here we will focus on the counterpart of $\mathcal{H}(s)$ and $\mathcal{L}(\Phi)$ spaces, whose reproducing kernel are of the form

$$
\frac{1-s(z) \overline{s(w)}}{1-z \bar{w}} \text { and } \frac{\Phi(z)+\overline{\Phi(w)}}{2(1-z \bar{w})}
$$

respectively.

These various definitions and corresponding results need to be adapted to the present case, where we do not have a law of composition. We note that the theory can be developed easily as in the classical way when the coefficients are real, but this is of course restrictive. On the other hand, the theory using Fueter variables works well because these variables are real when restricted to $x_{0}=0$ where $x_{0}$ denotes the real part of a quaternion.

There are important differences between the present treatment of Fueter hyperholomorphic functions and the treatment using Fueter variables; in the first case, the kernel functions are eigenvectors of the backward shift, in the case of Fueter variables the kernel functions are eigenvectors of the three underlying backward-shift operator. Here the kernel functions are not eigenvectors of the backward-shift operator.

However, the present approach allows to make connections with the theory of slice polyanalytic functions, in particular with slice hyperholomorphic functions, and will also allow a simpler functional calculus. Moreover, Toeplitz operators do appear in a natural way and play an important role.

In both cases, it is possible to develop a Schur type analysis. On the other hand, specific choices of the approach allow to make connections with slice hyperholomorphic functions. We here consider the cases

$$
h(x)=x_{1} \mathbf{e}_{1}+x_{2} \mathbf{e}_{2}+x_{3} \mathbf{e}_{3} \quad \text { and } \quad w(x)=(1-h(x))(1+h(x))^{-1}
$$

and relate the underlying analysis with the Appell polynomials setting. Note that $w(0)=1 \neq 0$. 
A key fact used in the paper is that the hyperholomorphic functions considered are of axial type, and hence uniquely determined by their values on the real line.

We shall prove that the $C K$-extension of $x_{1} \mathbf{e}_{1}+x_{2} \mathbf{e}_{2}+x_{3} \mathbf{e}_{3}$ is

$$
\begin{aligned}
& C K\left(x_{1} \mathbf{e}_{1}+x_{2} \mathbf{e}_{2}+x_{3} \mathbf{e}_{3}\right)=x_{1} \mathbf{e}_{1}+x_{2} \mathbf{e}_{2}+x_{3} \mathbf{e}_{3}+3 x_{0} \\
& \quad=\zeta_{1}(x) \mathbf{e}_{1}+\zeta_{2}(x) \mathbf{e}_{2}+\zeta_{3}(x) \mathbf{e}_{3}
\end{aligned}
$$

where $\zeta_{i}=x_{i}-x_{0} \mathbf{e}_{i}, i=1,2,3$ are the Fueter variables. Moreover we have

$$
\begin{aligned}
& C K\left(\left(x_{1} \mathbf{e}_{1}+x_{2} \mathbf{e}_{2}+x_{3} \mathbf{e}_{3}\right)^{m}\right) \odot C K\left(\left(x_{1} \mathbf{e}_{1}+x_{2} \mathbf{e}_{2}+x_{3} \mathbf{e}_{3}\right)^{n}\right) \\
& \quad=C K\left(\left(x_{1} \mathbf{e}_{1}+x_{2} \mathbf{e}_{2}+x_{3} \mathbf{e}_{3}\right)^{m+n}\right),
\end{aligned}
$$

so that we set

$$
C K\left(\left(x_{1} \mathbf{e}_{1}+x_{2} \mathbf{e}_{2}+x_{3} \mathbf{e}_{3}\right)^{m}\right)=\frac{Q_{m}(x)}{c_{m}} \stackrel{\text { def. }}{=} P_{m}(x),
$$

where $Q_{m}$ denotes the $m$-th quaternionic Appell polynomial (see [12, (3.8)] and [49]). The coefficients $c_{m}$ will be specified in Sect. 2. We are thus looking at a theory of hyperholomorphic functions of the variable

$$
P_{1}(x)=\zeta_{1}(x) \mathbf{e}_{1}+\zeta_{2}(x) \mathbf{e}_{2}+\zeta_{3}(x) \mathbf{e}_{3}=\frac{Q_{1}(x)}{c_{1}},
$$

equipped with the $C K$-product. In our discussion it is crucial that

$$
P_{1}^{n \odot}=P_{n} .
$$

We associate in a natural way to a Schur multiplier in the present setting a slice hyperholomorphic Schur multiplier; this allows to develop Schur analysis in the present setting.

The de Branges-Rovnyak space associated with a Schur multiplier $S$ allows in the cases considered up to now to get a coisometric realization of the multiplier. In the complex setting, this is the celebrated backwardshift realization (see [60]). Here, the situation is a bit different. We can still associate to $S$ a coisometric operator matrix, in the form (in the current setting) of the backward-shift realization, but the realization is on the level of the coefficients (like in [56] in the finite dimensional case).

We have given, or outlined, proofs of some classical results, for instance the extension result in Theorem 2.21 and the closely related Theorem 2.22. The reason is that the results play a key role in this paper and some of the arguments are not necessarily well known in the Clifford analysis community. We apply them in the quaternionic setting in particular in Theorem 4.21 and in Step 2 in the proof of Theorem 4.15.

The paper contains twelve sections, besides this Introduction. Section 2 contains some preliminary results. Section 3 contains results on reproducing kernel spaces and Toeplitz operators. In Sect. 4 we define the Hardy space in this framework, the backward-shift operator, Schur multipliers and their characterization. The Schur algorithm is presented in Sect. 5. Section 6 is focused on intrinsic functions, among which the polynomials $P_{n}$ and a description of Fueter hyperholomorphic functions of axial type which are also intrinsic. In Sect. 7 we consider de Branges-Rovnyak spaces while in Sect. 8 
we show how to define Blaschke functions, and the corresponding operator of multiplication which turns out to be an isometry. In Sect. 9 we consider the counterpart of Herglotz functions and multipliers and their associated kernels and Hilbert spaces. The next three sections concern the half-space case of Schur and Carathéodory multipliers. In Sect. 13 we summarize in a table a comparison between the various quaternionic settings.

\section{Preliminaries}

This section contains three subsections: the first one introduces the map $\chi$; the second one introduces the Fueter variables and the polynomials obtained via the Appell polynomials which will be the basis of our treatment. Finally, the third one shortly reviews positivity, analytic extensions and Toeplitz operators in the classical complex setting.

\subsection{Quaternions and the Map $\chi$}

We will work in the skew field of quaternions, which is defined to be

$$
\mathbb{H}=\left\{x=x_{0}+x_{1} \mathbf{e}_{1}+x_{2} \mathbf{e}_{2}+x_{3} \mathbf{e}_{3} \quad ; x_{0}, x_{1}, x_{2}, x_{3} \in \mathbb{R}\right\}
$$

where the imaginary units satisfy the multiplication rules $\mathbf{e}_{i}^{2}=-1, i=1,2,3$, $\mathbf{e}_{1} \mathbf{e}_{2}=-\mathbf{e}_{2} \mathbf{e}_{1}=\mathbf{e}_{3}, \mathbf{e}_{2} \mathbf{e}_{3}=-\mathbf{e}_{3} \mathbf{e}_{2}=\mathbf{e}_{1}, \mathbf{e}_{3} \mathbf{e}_{1}=-\mathbf{e}_{1} \mathbf{e}_{3}=\mathbf{e}_{2}$. The conjugate and the modulus of $x \in \mathbb{H}$ are defined by

$$
\bar{x}=\operatorname{Re}(x)-\underline{x} \quad \text { where } \quad \operatorname{Re}(x)=x_{0}, \quad \underline{x}=x_{1} \mathbf{e}_{1}+x_{2} \mathbf{e}_{2}+x_{3} \mathbf{e}_{3}
$$

and

$$
|x|=\sqrt{x \bar{x}}=\sqrt{x_{0}^{2}+x_{1}^{2}+x_{2}^{2}+x_{3}^{2}},
$$

respectively. The set of all imaginary units is given by $\mathbb{S}=\left\{q \in \mathbb{H} ; q^{2}=-1\right\}$. We note also that a domain $\Omega$ of $\mathbb{H}$ is called a slice domain if $\Omega \cap \mathbb{R}$ is nonempty and for all $I \in \mathbb{S}$, the set $\Omega_{I}:=\Omega \cap \mathbb{C}_{I}$ is a domain of the complex plane $\mathbb{C}_{I}$. If moreover, for every $x=u+I v \in \Omega$, the whole sphere

$$
[q]:=\{u+J v ; J \in \mathbb{S}\},
$$

is contained in $\Omega$, we say that $\Omega$ is an axially symmetric slice domain.

We can write a quaternion as $x=z+w \mathbf{e}_{2}$ with $z=x_{0}+x_{1} \mathbf{e}_{1}$ and $w=x_{2}+x_{3} \mathbf{e}_{1} \in \mathbb{C}$. The map $\chi$ defined by

$$
\chi\left(z+w \mathbf{e}_{2}\right)=\left(\begin{array}{cc}
z & w \\
-\bar{w} & \bar{z}
\end{array}\right)
$$

allows to transfer a number of problems from the quaternions to matrices in $\mathbb{C}^{2 \times 2}$. We recall the following result, whose proof is immediate and will be omitted.

Lemma 2.1. A matrix $M \in \mathbb{C}^{2 \times 2}$ belongs to the range of $\chi$ if and only if it satisfies the symmetry

$$
E^{-1} \bar{M} E=M
$$

where $E=\left(\begin{array}{cc}0 & 1 \\ -1 & 0\end{array}\right)$. 
For matrices and operators, there are various ways to define $\chi$. Let $X=A+B \mathbf{e}_{2} \in \mathbb{H}^{r \times s}$. We set

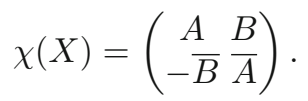

We define for a block matrix $\left(X_{j k}\right)$ with $X_{j k} \in \mathbb{H}^{r \times s}$,

$$
(\chi(X))_{j k}=\chi\left(X_{j k}\right) .
$$

For matrices $M_{1}, M_{2}$ possibly infinite, with block entries in $\mathbb{H}^{r \times s}$ and $\mathbb{H}^{s \times t}$, respectively, we have the property

$$
\chi\left(M_{1} M_{2}\right)=\chi\left(M_{1}\right) \chi\left(M_{2}\right) .
$$

We note that $\chi$ will not be compatible with the $C K$-product. An important tool in the paper consists of bounded block Toeplitz operators, with blocks in the range of $\chi$ :

$$
T=\left(\chi\left(X_{j-k}\right)\right)_{j, k=0}^{\infty} .
$$

and we will need the following result, set for general operators.

Proposition 2.2. The operator $\tau$

$$
\tau=\left(X_{j k}\right)_{j, k=0}^{\infty}
$$

is bounded from $\ell_{2}\left(\mathbb{N}_{0}, \mathbb{H}^{s}\right)$ into $\ell_{2}\left(\mathbb{N}_{0}, \mathbb{H}^{r}\right)$ if and only if the operator $T$ defined by

$$
T=\left(\chi\left(X_{j k}\right)\right)_{j, k=0}^{\infty},
$$

where the $X_{j k}$ are matrices in $\mathbb{H}^{r \times s}$ is bounded from $\ell_{2}\left(\mathbb{N}_{0}, \mathbb{C}^{2 s}\right)$ into $\ell_{2}\left(\mathbb{N}_{0}, \mathbb{C}^{2 r}\right)$, and both operators have same norm.

Proof. One direction is clear: If $\tau$ is bounded, there is a constant $K>0$ such that

$$
\sum_{i, j, k=0}^{\infty} q_{i}^{*} X_{i j} X_{j k}^{*} q_{k} \leq K \sum_{i=0}^{\infty} q_{i}^{*} q_{i}, \quad \text { for any } q_{i} \in \mathbb{H}^{r} .
$$

Applying $\chi$ we get

$$
\sum_{i, j, k=0}^{\infty} \chi\left(q_{i}\right)^{*} \chi\left(X_{i j}\right) \chi\left(X_{j k}\right)^{*} \chi\left(q_{k}\right) \leq K \chi\left(\sum_{i=0}^{\infty} q_{i}^{*} q_{i} I_{2}\right),
$$

where $I_{2}$ denotes the identity matrix of order 2. Multiplying this inequality by $\left(\begin{array}{ll}1 & 0\end{array}\right)$ on the left and by its transpose on the right, we get the result. $\in \mathbb{C}^{r}$

Conversely, if $T$ is bounded there exists $K>0$ such that for all $u_{1}, v_{1}, \ldots$

$$
\sum_{i, j, k=0}^{\infty}\left(u_{i}^{*} v_{i}^{*}\right) \chi\left(X_{i j}\right) \chi\left(X_{j k}\right)^{*}\left(\begin{array}{l}
u_{k} \\
v_{k}
\end{array}\right) \leq K\left(\sum_{i=0}^{\infty} u_{i}^{*} u_{i}+v_{i}^{*} v_{i}\right)
$$

and

$$
\sum_{i, j, k=0}^{\infty}\left(-v_{i}^{t} u_{i}^{t}\right) \chi\left(X_{i j}\right) \chi\left(X_{j k}\right)^{*}\left(\begin{array}{c}
-\overline{v_{k}} \\
\overline{u_{k}}
\end{array}\right) \leq K\left(\sum_{i=0}^{\infty} u_{i}^{*} u_{i}+v_{i}^{*} v_{i}\right)
$$


Set

$$
\begin{aligned}
e^{*} & =\left(u_{1}^{*} v_{1}^{*} u_{2}^{*} v_{2}^{*} \cdots\right) \\
f^{*} & =\left(-v_{1}^{t} u_{1}^{t}-v_{2}^{t} u_{2}^{t} \cdots\right)
\end{aligned}
$$

and denote by $M$ the bounded linear operator with $i, k$ block equal to $M_{i k}=$ $\chi\left(Y_{i k}\right)$, with $Y_{i k}=\sum_{j=1}^{\infty} X_{i j} X_{j k}^{*}, i, j=1, \ldots$ We have

$$
\left(\begin{array}{l}
e^{*} \\
f^{*}
\end{array}\right) M(e f)=\left(\begin{array}{ll}
e^{*} M e & e^{*} M f \\
f^{*} M e & f^{*} M f
\end{array}\right) \leq\|M\|\left(e^{*} e+f^{*} f\right) I_{2} .
$$

On the other hand, setting

$$
q_{i}^{*}=u_{i}^{*}+v_{i}^{*} \mathbf{e}_{2}, \quad i=1,2, \ldots
$$

we rewrite $(2.6)-(2.7)$ as

$$
\left(\begin{array}{c}
e^{*} \\
f^{*}
\end{array}\right) M(e f)=\left(\chi\left(q_{1}\right)^{*} \chi\left(q_{2}\right)^{*} \ldots\right)\left(\begin{array}{ccc}
\chi\left(Y_{11}\right) & \chi\left(Y_{12}\right) & \ldots \\
\chi\left(Y_{12}\right)^{*} & \chi\left(Y_{22}\right) & \cdots \\
\vdots & & \ddots
\end{array}\right)\left(\begin{array}{c}
\chi\left(q_{1}\right) \\
\chi\left(q_{2}\right) \\
\vdots
\end{array}\right)
$$

Comparing with (2.8) we get

$$
\left(\chi\left(q_{1}\right)^{*} \chi\left(q_{2}\right)^{*} \ldots\right)\left(\begin{array}{ccc}
\chi\left(Y_{11}\right) & \chi\left(Y_{12}\right) & \cdots \\
\chi\left(Y_{12}\right)^{*} & \chi\left(Y_{22}\right) & \cdots \\
\vdots & & \ddots
\end{array}\right)\left(\begin{array}{c}
\chi\left(q_{1}\right) \\
\chi\left(q_{2}\right) \\
\vdots
\end{array}\right) \leq\|M\| \chi\left(e^{*} e+f^{*} f\right)
$$

and hence the result, since $\chi$ preserves order.

The claim on the norms being the same follows from the previous inequality and (2.5).

\subsection{Various Notions of Hyperholomorphy and Homogeneous Polynomials}

In this section we briefly review the setting of Fueter variables and the Cauchy-Kovalevskaya product. We recall that left-hyperholomorphic functions (we will usually just say hyperholomorphic in the sequel) are solutions of the equation $D f=0$, where $D$ denotes the Cauchy-Fueter operator

$$
D=\frac{\partial}{\partial x_{0}}+\mathbf{e}_{1} \frac{\partial}{\partial x_{1}}+\mathbf{e}_{2} \frac{\partial}{\partial x_{2}}+\mathbf{e}_{3} \frac{\partial}{\partial x_{3}} .
$$

These functions are widely studied in the literature. They are, in particular, harmonic functions in four real variables. Unfortunately, the monomials $x^{n}$ in the quaternionic variable $x$ are not in the kernel of the Cauchy-Fueter operator, not even when $n=1$. However, hyperholomorphic functions admit a series expansion in terms of the so-called Fueter variables, as we shall see below. We point out that, in this paper, we shall provide only the notions and results needed in the sequel, and for further information on this class of functions we refer the reader to $[38,63]$.

In view of the Cauchy-Kovalevskaya theorem, a linear system of first order differential equations satisfied by the real components of $f$ has a unique solution when the function $\varphi\left(x_{1}, x_{2}, x_{3}\right)=f\left(0, x_{1}, x_{2}, x_{3}\right)$ is pre-assigned (and assumed real analytic). The function $f$ with this initial condition and solution 
of $D f=0$ is called the Cauchy-Kovalevskaya extension of $\varphi$ (here written as $C K(\varphi)$ and abbreviated as $C K$-extension).

Among the important solutions of the equation $D f=0$ there are the Fueter variables

$$
\zeta_{j}(x)=x_{j}-\mathbf{e}_{j} x_{0}, \quad j=1,2,3,
$$

corresponding respectively to $\varphi_{j}(x)=x_{j}, j=1,2,3$, and their symmetric products

$$
\zeta^{\nu}=\zeta_{1}^{\nu_{1} \times} \times \zeta_{2}^{\nu_{2} \times} \times \zeta_{3}^{\nu_{3} \times}, \quad \nu=\left(\nu_{1}, \nu_{2}, \nu_{3}\right) \in \mathbb{N}_{0}^{3},
$$

with, for $a_{1}, \ldots, a_{n} \in \mathbb{H}$

$$
a_{1} \times a_{2} \times \cdots \times a_{n}=\frac{1}{n !} \sum_{\sigma \in S_{n}} a_{\sigma(1)} a_{\sigma(2)} \cdots a_{\sigma(n)} .
$$

We note that $\zeta^{\nu}=C K\left(x^{\nu}\right)$ with $x^{\nu}=x_{1}^{\nu_{1}} x_{2}^{\nu_{2}} x_{3}^{\nu_{3}}$. A direct proof that $\zeta^{\nu}$ given by (2.11) is hyperholomorphic (and hence is the $C K$-extension of $x^{\nu}$ ) is not trivial and can be found in [46, Sect. 3], [59]. The argument works also in the split quaternion setting. See [18, p. 333-334]. It is important to note that every function hyperholomorphic in a neighborhood of the origin can be written as a convergent power series in the form of a Fueter series

$$
f(x)=\sum_{\nu \in \mathbb{N}_{0}^{3}} \zeta^{\nu} f_{\nu}
$$

where the coefficients $f_{\nu}$ belong to $\mathbb{H}$. See [32]. A proof based on the Gleason problem can be found in [23].

Using the CK-extension one can define a product that preserves the hyperholomorphicity, the so-called CK-product denoted by $\odot$. The idea to compute the CK-product is the following: if $f$ and $g$ are two hyperholomorphic functions, we take their restriction to $x_{0}=0$, which are real analytic functions, and consider their pointwise multiplication. Then, we take the Cauchy-Kowalevskaya extension of this pointwise product, which exists and is unique, to define

$$
f \odot g=C K\left(f\left(0, x_{1}, x_{2}, x_{3}\right) g\left(0, x_{1}, x_{2}, x_{3}\right)\right),
$$

see [63]. Moreover, we note that the following formula holds

$$
C K[\varphi(\underline{x})](x)=\exp \left(-x_{0} \partial_{\underline{x}}\right)[\varphi(\underline{x})](x) .
$$

For power series of the form (2.12) the $C K$-product is a convolution on the coefficients along the basis $\zeta^{\nu}$, and in particular

$$
\zeta^{\nu} p \odot \zeta^{\mu} q=\zeta^{\nu+\mu} p q, \quad p, q \in \mathbb{H}, \quad \mu, \nu \in \mathbb{N}_{0}^{3},
$$

where $\nu+\mu$ is defined componentwise, see [32,82].

We now turn to a bound for the $C K$-product; see also [23, pp. 132-133].

Lemma 2.3. Let $\rho>0$. There exists $\epsilon>0$ such that:

$$
x_{0}^{2}+x_{j}^{2}<\epsilon, \quad j=1,2,3, \Longrightarrow \sum_{\substack{\alpha \in \mathbb{N}_{0}^{3} \\ \alpha \neq(0,0,0)}}\left|\zeta(x)^{\alpha}\right|\left|f_{\alpha}\right|<\rho .
$$


Then

$$
\left|\left(\sum_{\substack{\alpha \in \mathbb{N}_{0}^{3} \\ \alpha \neq(0,0,0)}} \zeta(x)^{\alpha} f_{\alpha}\right)^{\odot n}\right|<\rho^{n}, \quad n=1,2,3, \ldots
$$

Proof. We first note that for $\nu \in \mathbb{N}_{0}^{3}$

$$
\left|\zeta^{\nu}(x)\right| \leq \epsilon^{|\nu|}, \quad \text { where } \quad x_{0}^{2}+x_{j}^{2}<\epsilon, \quad j=1,2,3 .
$$

The existence of $\epsilon$ follows from the dominated convergence theorem, and the first assertion follows. Then, setting $g(x)=\sum_{\beta \in \mathbb{N}_{0}^{3}} \zeta(x)^{\beta} g_{\beta}$ we have

$$
\begin{aligned}
|(f \odot g)(x)| & \leq\left|\sum_{\gamma \in \mathbb{N}_{0}^{3}}\right| \zeta^{\gamma}(x)|\cdot| \sum_{\substack{\alpha, \beta \in \mathbb{N}_{0}^{3} \\
\alpha+\beta=\gamma}} f_{\alpha} g_{\beta} \mid \\
& \leq \sum_{\alpha, \beta \in \mathbb{N}_{0}^{3}} \epsilon^{|\alpha|+|\beta|}\left|f_{\alpha}\right| \cdot\left|g_{\beta}\right| \\
& \leq\left(\sum_{\alpha \in \mathbb{N}_{0}^{3}} \epsilon^{|\alpha|}\left|f_{\alpha}\right|\right)\left(\sum_{\beta \in \mathbb{N}_{0}^{3}} \epsilon^{|\beta|}\left|b_{\beta}\right|\right),
\end{aligned}
$$

from which (2.15) follows.

Lemma 2.4. The $C K$-extension of $x_{1} \mathbf{e}_{1}+x_{2} \mathbf{e}_{2}+x_{3} \mathbf{e}_{3}$ to a Fueter hyperholomorphic function is

$$
x_{1} \mathbf{e}_{1}+x_{2} \mathbf{e}_{2}+x_{3} \mathbf{e}_{3}+3 x_{0}=\zeta_{1}(x) \mathbf{e}_{1}+\zeta_{2}(x) \mathbf{e}_{2}+\zeta_{3}(x) \mathbf{e}_{3}
$$

where $\zeta_{1}, \zeta_{2}, \zeta_{3}$ are the Fueter variables.

Proof. It suffices to note that the function

$$
\begin{aligned}
\zeta_{1}(x) \mathbf{e}_{1}+\zeta_{2}(x) \mathbf{e}_{2}+\zeta_{3}(x) \mathbf{e}_{3} & =\left(x_{1}-\mathbf{e}_{1} x_{0}\right) \mathbf{e}_{1}+\left(x_{2}-\mathbf{e}_{2} x_{0}\right) \mathbf{e}_{2}+\left(x_{3}-\mathbf{e}_{3} x_{0}\right) \mathbf{e}_{3} \\
& =x_{1} \mathbf{e}_{1}+x_{2} \mathbf{e}_{2}+x_{3} \mathbf{e}_{3}+3 x_{0}
\end{aligned}
$$

is Fueter hyperholomorphic and its restriction to $x_{0}=0$ is the given function.

Let us now introduce another type of Fueter hyperholomorphic homogeneous polynomials, see $[33,49,53]$ :

Definition 2.5. The polynomials

$$
Q_{m}(x)=\sum_{j=0}^{m} T_{j}^{m} x^{m-j} \bar{x}^{j}
$$

where

$$
T_{j}^{m}=\frac{2(m-j+1)}{(m+1)(m+2)}, \quad m=0,1, \ldots,
$$

are called the $m$-th quaternionic Appell polynomials. 
The polynomials $\left(Q_{m}\right)_{m \geq 0}$ are Fueter regular. Moreover, a generalized Fueter regular exponential function associated to these polynomials was considered in the literature, see for example [33]. Another interesting feature of the quaternionic Appell polynomials is that they can be obtained by applying the Fueter mapping applied to the standard quaternionic monomials $x^{m}$. In particular, in [49] the following formula is proved

$$
Q_{m}(x)=-\frac{\Delta\left(x^{m+2}\right)}{2(m+1)(m+2)}, \quad m=0,1, \ldots
$$

We then define another kind of Fueter hyperholomorphic polynomials by

$$
P_{m}(x) \stackrel{\text { def. }}{=} \frac{Q_{m}(x)}{c_{m}},
$$

where

$$
c_{m}=\sum_{j=0}^{m}(-1)^{j} T_{j}^{m} .
$$

We have the following relation between the polynomials $P_{m}$ and the $C K$-extension of $x_{1} \mathbf{e}_{1}+x_{2} \mathbf{e}_{2}+x_{3} \mathbf{e}_{3}$ in (2.17):

Proposition 2.6. The following equality holds:

$$
C K\left(\left(x_{1} \mathbf{e}_{1}+x_{2} \mathbf{e}_{2}+x_{3} \mathbf{e}_{3}\right)^{m}\right)=P_{m}(x) .
$$

Proof. The proof is simple and it is based on the fact that at both hand sides there are monogenic functions which coincide on $x_{0}=0$ :

$$
\left(x_{1} \mathbf{e}_{1}+x_{2} \mathbf{e}_{2}+x_{3} \mathbf{e}_{3}\right)^{m}=\frac{1}{c_{m}} \sum_{j=0}^{m} T_{j}^{m} \underline{x}^{m-j}(-\underline{x})^{j}=\frac{1}{c_{m}} \sum_{j=0}^{m}(-1)^{j} T_{j}^{m} \underline{x}^{m}=\underline{x}^{m} .
$$

Remark 2.7. The polynomials $Q_{m}$ are called Appell since they satisfy the Appell property

$$
\frac{1}{2} \bar{D} Q_{m}=m Q_{m-1}, \quad m \geq 1
$$

the $P_{m}$ do not respect such a property, since

$$
\frac{1}{2} \bar{D} P_{m}=m \frac{c_{m-1}}{c_{m}} P_{m-1}, \quad m \geq 1,
$$

however, they behave better with respect to the $C K$-product, as we shall see below. In particular, for even indexes of the form $m=2 k$, the Appell property is still satisfied by the polynomials $\left(P_{2 k}\right)_{k \geq 0}$ since we have $c_{m-1}=c_{m}$ in this case.

In what follows, we are looking at a theory of hyperholomorphic functions of the variable

$$
P_{1}(x)=\frac{Q_{1}(x)}{c_{1}}=\zeta_{1}(x) \mathbf{e}_{1}+\zeta_{2}(x) \mathbf{e}_{2}+\zeta_{3}(x) \mathbf{e}_{3},
$$

with the $C K$-product. Moreover, note that

$$
P_{1}\left(x_{0}\right)=3 x_{0} \text {. }
$$


The $\odot$-product is not a convolution on the coefficients of the $P_{n}$ : in opposition to (2.14) we have, in general,

$$
P_{n} p \odot P_{m} q \neq P_{n+m} p q, \quad n, m \in \mathbb{N}, \quad p, q \in \mathbb{H} .
$$

In particular, in general

$$
\left(1-P_{1} q\right)^{-\odot} \neq \sum_{n=0}^{\infty} P_{n} q^{n}
$$

for $q \in \mathbb{H}$ in a neighborhood of the origin.

This obstruction is the source of the main difficulties and new results in the present paper. Still, we have the following simple result, which plays a key role in the computations [see in particular (3.11)].

Lemma 2.8. It holds that

$$
\left(P_{n} \odot P_{m}\right)(x)=P_{n+m}(x),
$$

and, in particular,

$$
P_{n}(x)=\left(P_{1}(x)\right)^{\odot n}, \quad n=1,2, \ldots
$$

Furthermore, for $n, m, k \in \mathbb{N}_{0}$ and $u \in \mathbb{H}^{r}$

$$
\left(P_{n} \odot\left(P_{m} \odot P_{k} u\right)\right)=P_{n+m+k} u=P_{n+m} \odot P_{k} u .
$$

Proof. We have

$$
\begin{aligned}
& C K\left(\left(x_{1} \mathbf{e}_{1}+x_{2} \mathbf{e}_{2}+x_{3} \mathbf{e}_{3}\right)^{n}\right) \odot C K\left(\left(x_{1} \mathbf{e}_{1}+x_{2} \mathbf{e}_{2}+x_{3} \mathbf{e}_{3}\right)^{m}\right) \\
& \quad=C K\left(\left(x_{1} \mathbf{e}_{1}+x_{2} \mathbf{e}_{2}+x_{3} \mathbf{e}_{3}\right)^{n+m}\right) \\
& \quad=\left(\zeta_{1}(x) \mathbf{e}_{1}+\zeta_{2}(x) \mathbf{e}_{2}+\zeta_{3}(x) \mathbf{e}_{3}\right)^{\odot(n+m)},
\end{aligned}
$$

where we used (2.20) in the last equality. In particular, by iteration, we obtain (2.26). The last claim follows from restricting the equalities for $x_{0}=0$, and checking that they are equal to $\left(x_{1} \mathbf{e}_{1}+x_{2} \mathbf{e}_{2}+x_{3} \mathbf{e}_{3}\right)^{n+m+k} u$.

Corollary 2.9. For every $\rho>0$ there exists $\epsilon>0$ such that

$$
x_{0}^{2}+x_{j}^{2}<\epsilon, \quad j=1,2,3, \quad \Longrightarrow \quad\left|P_{n}(x)\right|<\rho^{n} .
$$

Proof. This follows by induction from Lemma 2.3 with $f=P_{1}$ and $g=P_{n}$, $n \in \mathbb{N}$.

We have (see e.g. [23, (2.19) p. 135] with $A_{u}=\mathbf{e}_{u}, u=1,2,3$ )

$$
\left(\zeta_{1}(x) \mathbf{e}_{1}+\zeta_{2}(x) \mathbf{e}_{2}+\zeta_{3}(x) \mathbf{e}_{3}\right)^{m \odot}=\sum_{|\nu|=m} \zeta^{\nu} \mathbf{e}^{\nu} \frac{|\nu| !}{\nu !}
$$

where $\zeta^{\nu}$ is defined in $(2.11)$.

Remark 2.10. One could take the $C K$-extension of another linear combination such as $t_{1} x_{1} \mathbf{e}_{1}+t_{2} x_{2} \mathbf{e}_{2}+t_{3} x_{3} \mathbf{e}_{3}$, namely

$$
\begin{aligned}
& t_{1} \zeta_{1} \mathbf{e}_{1}+t_{2} \zeta_{2} \mathbf{e}_{2}+t_{3} \zeta_{3} \mathbf{e}_{3} \\
& \quad=t_{1} x_{1} \mathbf{e}_{1}+t_{2} x_{2} \mathbf{e}_{2}+t_{3} x_{3} \mathbf{e}_{3}+\left(t_{1}+t_{2}+t_{3}\right) x_{0}
\end{aligned}
$$

and develop a similar theory. 
Let $f\left(x_{0}\right)=\sum_{n=0}^{\infty} x_{0}^{n} a_{n}$ (with $\left.a_{0}, a_{1}, \ldots \in \mathbb{H}\right)$ be a real analytic function near the origin. It does not have a unique hyperholomorphic extension of course, as seen by taking

$$
\zeta_{1}(x) \mathbf{e}_{1} \text { and } \zeta_{2}(x) \mathbf{e}_{2},
$$

in fact both functions are equal to $x_{0}$ on the real line. However the extension becomes unique by requiring that it is of a special form:

Lemma 2.11. Let $f\left(x_{0}\right)=\sum_{n=0}^{\infty} x_{0}^{n} a_{n}, a_{n} \in \mathbb{H}$ be a real analytic function near the origin. It has a unique (left) hyperholomorphic extension of the form $f(x)=\sum_{n=0}^{\infty} P_{n}(x) b_{n}$, namely

$$
f(x)=\sum_{n=0}^{\infty} P_{n}(x) \frac{a_{n}}{3^{n}} .
$$

Similarly, its unique right hyperholomorphic extension is

$$
g(x)=\sum_{n=0}^{\infty} \frac{a_{n}}{3^{n}} P_{n}(x) .
$$

Proof. The function $f(x)$ is indeed an extension of the required form. If there is another one, say $\tilde{f}(x)=\sum_{n=0}^{\infty} P_{n}(x) d_{n}$ we get when setting $x_{1}=x_{2}=$ $x_{3}=0$

$$
\sum_{n=0}^{\infty}\left(3 x_{0}\right)^{n} b_{n}=\sum_{n=0}^{\infty}\left(3 x_{0}\right)^{n} d_{n}
$$

and so $b_{n}=d_{n}, n=0,1, \ldots$ A similar reasoning works for $g$.

Remark 2.12. We note that the polynomials $P_{m}$ and $Q_{m}$ are both left and right hyperholomorphic and in fact $P_{m}(x)$ corresponds to both the left and right CK-extension of $\left(x_{1} \mathbf{e}_{1}+x_{2} \mathbf{e}_{2}+x_{3} \mathbf{e}_{3}\right)^{m}$.

Actually, the previous result is a particular case of a more general result that holds for Fueter hyperholomorphic functions of axial type, whose definition which comes from the more general case of axially monogenic functions, see [47], is the following:

Definition 2.13. A Fueter hyperholomorphic function is of axial type (or axially hyperholomorphic) if it is of the form

$$
A\left(x_{0},|\underline{x}|\right)+\frac{\underline{x}}{|\underline{x}|} B\left(x_{0},|\underline{x}|\right),
$$

where $A, B$ are quaternionic valued.

The condition that a function $f$ of axial type is in the kernel of the Cauchy-Fueter operator $D$ translates into the Vekua system

$$
\partial_{x_{0}} A-\partial_{\rho} B=\frac{2}{\rho} B, \quad \partial_{x_{0}} B+\partial_{\rho} A=0, \quad \rho=|\underline{x}| .
$$

Starting from any real analytic function $A\left(x_{0}\right)$ it is possible to construct its unique Fueter hyperholomorphic extension of axial type.

We will say that a matrix-valued hyperholomorphic function is of axial type if all its entries, as matrix, are of axial type. 
Remark 2.14. Functions of the form (2.30) are quaternionic special monogenic according to the terminology in [3]. Any quaternionic special monogenic function in a neighborhood of the origin is of axial type. In fact any polynomial $P_{m}(x)$ is the sum of terms of the form

$$
\left(x_{0}^{2}+|\underline{x}|^{2}\right)^{k}\left(x_{0} \pm \underline{x}\right)^{h}, \quad 2 k+h=m, k \geq 0, h \geq 0
$$

which are evidently of axial type. This fact was already noted in [33], Property 2. A Fueter regular function represented by a uniformly convergent series of the form (2.30) is such that

$$
\begin{aligned}
\sum_{m \geq 0} A_{m}\left(x_{0},|\underline{x}|\right)+\frac{\underline{x}}{|\underline{x}|} B_{m}\left(x_{0},|\underline{x}|\right) & =\sum_{m \geq 0} A_{m}\left(x_{0},|\underline{x}|\right)+\frac{\underline{x}}{|\underline{x}|} \sum_{m \geq 0} B_{m}\left(x_{0},|\underline{x}|\right) \\
& =A\left(x_{0},|\underline{x}|\right)+\frac{\underline{x}}{|\underline{x}|} B\left(x_{0},|\underline{x}|\right)
\end{aligned}
$$

where the pair $A, B$ satisfy the Vekua system. Conversely, any function of axial type is of the form (2.30), by Theorem 3.10 in [12].

We recall the notion of slice polyanalytic functions, see [14].

Definition 2.15. A real differentiable function $f: \Omega \longrightarrow \mathbb{H}$ of the form

$$
f(x)=\alpha(u, v)+I \beta(u, v), \quad x=u+I v \in \Omega
$$

with $\alpha(u,-v)=\alpha(u, v)$ and $\beta(u,-v)=-\beta(u, v)$ is called left slice polyanalytic of order $N$, if for all $I \in \mathbb{S}, f_{I}$ is left polyanalytic of order $N$ on $\Omega_{I}$, namely if

$$
\bar{\partial}_{I}^{N} f(u+I v):=\frac{1}{2^{N}}\left(\frac{\partial}{\partial u}+I \frac{\partial}{\partial v}\right)^{N} f_{I}(u+I v)=0 .
$$

When $N=1$ the notion coincides with that one of slice hyperholomorphicity (slice regularity).

We have the following characterization, see [14, Proposition 3.6]:

Proposition 2.16. A function $f$ defined on a domain $\Omega \subseteq \mathbb{H}$ is slice polyanalytic of order $N$ on $\Omega$ if and only if it can be written as

$$
f(x):=\sum_{k=0}^{N-1} \bar{x}^{k} f_{k}(x)
$$

where $f_{0}, \ldots, f_{N-1}$ are slice regular functions in $\Omega$.

As a consequence:

Corollary 2.17. The polynomial $P_{m}$ is slice polyanalytic of order $m+1$.

Proof. For all $0 \leq k \leq m$, we set $f_{k}(x)=\frac{T_{k}^{m}}{c_{m}} x^{m-k}$. It is clear that all $f_{k}$ are slice regular functions on $\Omega$, being polynomials in the variable $x$. Moreover, we note that

$$
P_{m}(x)=\sum_{k=0}^{m} \bar{x}^{k} f_{k}(x), \forall x \in \Omega .
$$

Hence, the thesis follows using Proposition 2.16. 
In the definition of the polynomials $P_{m}$ we note that to write the monomials as $x^{j} \bar{x}^{\ell}$ or $\bar{x}^{\ell} x^{j}$ does not make any difference since $x \bar{x}=\bar{x} x$.

A Representation Formula for Fueter hyperholomorphic functions of axial type is immediately deduce from the fact that they are slice functions, see [62], so we have:

Proposition 2.18. Let $f: \Omega \subset \mathbb{H} \rightarrow \mathbb{H}$ be a Fueter hyperholomorphic function of axial type where $\Omega$ is an axially symmetric slice domain. Let $J \in \mathbb{S}$, then for any $x=u+I_{x} v \in \Omega$ the following equality holds :

$f\left(u+I_{x} v\right)=\frac{1}{2}\left[f_{J}(u+J v)+f_{J}(u-J v)\right]+\frac{I_{x} J}{2}\left[f_{J}(u-J v)-f_{J}(u+J v)\right]$.

Proof. We note that the Fueter hyperholomorphic polynomials $\left(P_{m}\right)_{m \geq 0}$ are slice polyanalytic of order $m+1$ thanks to Corollary 2.17. Thus, the Representation Formula is an immediate consequence.

Remark 2.19. An alternative proof of the previous Representation Formula in the Fueter hyperholomorphic context consists to apply Proposition 3.13 in [14] to each polynomial $P_{m}$.

We conclude this part with a result which will be used in the sequel while dealing with kernels:

Proposition 2.20. The polynomial $\overline{P_{m}(x)}$ is right anti-hyperholomorphic in $x$, namely it satisfies

$$
\overline{P_{m}(x)} \bar{D}=\frac{\partial \overline{P_{m}}}{\partial x_{0}}-\frac{\partial \overline{P_{m}}}{\partial x_{1}} \mathbf{e}_{1}-\frac{\partial \overline{P_{m}}}{\partial x_{2}} \mathbf{e}_{2}-\frac{\partial \overline{P_{m}}}{\partial x_{3}} \mathbf{e}_{3}=0 .
$$

More in general, if $f, g$ are left hyperholomorphic, $\overline{f \odot g}=\bar{g} \odot_{R} \bar{f}$ is right anti-hyperholomorphic.

Proof. We immediately have:

$$
\overline{P_{m}(x)} \bar{D}=\overline{D P_{m}(x)}=0,
$$

and the first assertion follows. Then we have

$$
\left.\overline{f \odot g}=\overline{C K\left(f_{\mid x_{0}=0} \odot g_{\mid x_{0}=0}\right.}\right)=C K\left(\overline{f_{\mid x_{0}=0} \odot g_{\mid x_{0}=0}}\right) .
$$

We now note that

$$
\begin{aligned}
C K\left(\overline{f_{\mid x_{0}=0} \odot g_{\mid x_{0}=0}}\right) & =C K\left(\overline{g_{\mid x_{0}=0}} \odot_{R} \overline{f_{\mid x_{0}=0}}\right) \\
& =C K\left(\overline{g_{\mid x_{0}=0}}\right) \odot_{R} C K\left(\overline{f_{\mid x_{0}=0}}\right)=\bar{g} \odot_{R} \bar{f}
\end{aligned}
$$

which concludes the proof.

\subsection{Positivity, Analytic Extension and Toeplitz Operators}

This section considers the complex variable setting. Recall first that a $\mathbb{C}^{n \times n}$ valued function $K(z, w)$ defined for $z, w$ varying in some set $\Omega$ is called positive definite if for every choice of $N \in \mathbb{N}_{0}$ and $w_{1}, \ldots, w_{N} \in \Omega$ the block matrix $\left(K\left(w_{j}, w_{k}\right)\right)_{j, k=1}^{N}$ is non-negative. Associated to $K(z, w)$ is a uniquely defined Hilbert space of $\mathbb{C}^{n}$-valued functions defined on $\Omega$, denoted here $\mathcal{H}(K)$, and with the properties: 
(1) For every $c \in \mathbb{C}^{n}$ and $w \in \Omega$, the function $K_{w} c: z \mapsto K(z, w) c$ belongs to $\mathcal{H}(K)$, and

(2) For every $f \in \mathcal{H}(K)$ and $w, c$ as above,

$$
\left\langle f, K_{w} c\right\rangle=c^{*} f(w) .
$$

$\mathcal{H}(K)$ is called the reproducing kernel Hilbert space with reproducing kernel $K(z, w)$, and there is a one-to-one correspondence between reproducing kernel Hilbert spaces and positive definite functions; see $[26,75,79]$. We recall the following result, which originates with the work of Donoghue [50]. We take a real neighborhood of the origin, but it could be replaced by any other zero set in the open unit disk.

Theorem 2.21. Let $s$ be a $\mathbb{C}^{r \times t}$-valued function defined in a neighborhood $(-\epsilon, \epsilon)$ of the origin, and such that the kernel

$$
\frac{I_{r}-s(a) s(b)^{*}}{1-a b}
$$

is positive definite in $(-\epsilon, \epsilon)$. Then s has a (uniquely defined) analytic and contractive extension to the open unit disk.

Proof. The proof can be found in e.g. [6, pp. 45-46]. For completeness we outline it. We set $r=s=1$ to simplify the notation. Let $\rho_{w}(z)=1-$ $z \bar{w}$. The function $1 / \rho_{w}(z)$ is positive definite in the open unit disk $\mathbb{D}$, with reproducing kernel Hilbert space the Hardy space of the open unit disk, denoted $\mathbf{H}_{2}(\mathbb{D})$, and consisting of the power series $f(z)=\sum_{n=0}^{\infty} a_{n} z^{n}$ with complex coefficients satisfying $\|f\|_{2}^{2} \stackrel{\text { def. }}{=} \sum_{n=0}^{\infty}\left|a_{n}\right|^{2}<\infty$. The formula

$$
T\left(1 / \rho_{x}\right)=\frac{\overline{s(x)}}{\rho_{x}}, \quad x \in(-1 / 3,1 / 3)
$$

extends linearly to a densely defined operator $T$ from $\mathbf{H}_{2}(\mathbb{D})$ into itself. The positivity of the kernel (2.33) and the definition of the inner product in the Hardy space implies that $T$ is a contraction, and hence extends to an everywhere defined contraction, still denoted by $T$, from $\mathbf{H}_{2}(\mathbb{D})$ into itself. Let $f \in \mathbf{H}_{2}(\mathbb{D})$. The adjoint of $T$ satisfies:

$$
\left(T^{*} f\right)(x)=\left\langle T^{*} f, \frac{1}{\rho_{x}}\right\rangle=\left\langle f, \frac{\overline{s(x)}}{\rho_{x}}\right\rangle=s(x) f(x), \quad x \in(-1 / 3,1 / 3) .
$$

Take first $f(z)=1$. Since $T^{*} 1$ is analytic in the open unit disk, it is an analytic extension of $s(x), x \in(-1 / 3,1 / 3)$. It remains to check that $T^{*} 1$ is contractive in $\mathbb{D}$. Equation (2.34) extends analytically to

$$
\left(T^{*} f\right)(z)=\left(T^{*} 1\right)(z) f(z), \quad z \in \mathbb{D} .
$$

So $T^{*}$ is the operator of multiplication by $T^{*} 1$. Since it is bounded, the formula for the adjoint of a multiplication operator acting in a reproducing kernel Hilbert space gives

$$
T^{*}\left(1 / \rho_{w}\right)=\frac{\overline{T^{*} 1(w)}}{\rho_{w}} .
$$


Since it is contractive, writing that $\left\|T^{*} 1 / \rho_{w}\right\| \leq\left\|1 / \rho_{w}\right\|$ we get

$$
\frac{\left|T^{*} 1(w)\right|^{2}}{1-|w|^{2}} \leq \frac{1}{1-|w|^{2}}, \quad w \in \mathbb{D},
$$

and hence $T^{*} 1$ takes contractive values in the open unit disk.

We also recall (we refer to [76] for more information on Toeplitz operators):

Theorem 2.22. Let $s$ be $a \mathbb{C}^{r \times t}$-valued function analytic in the open unit disk, with power series expansion $s(z)=\sum_{n=0}^{\infty} s_{n} z^{n}$. Then, $s$ is contractive in the open unit disk if and only if the lower triangular block-Toeplitz operator

$$
T_{s}=\left(\begin{array}{cccc}
s_{0} & 0 & 0 & \cdots \\
s_{1} & s_{0} & 0 & \cdots \\
s_{2} & s_{1} & s_{0} & 0 \\
\vdots & \ddots & \ddots & \ddots
\end{array}\right)
$$

is a contraction from $\ell_{2}\left(\mathbb{N}_{0}, \mathbb{C}^{t}\right)$ into $\ell_{2}\left(\mathbb{N}_{0}, \mathbb{C}^{r}\right)$.

Proof. We set $r=t=1$ to simplify the arguments. Assume first that $s$ is a contraction, and let $P$ denote the orthogonal projection from $\mathbf{L}_{2}(\mathbb{T})$ onto $\mathbf{H}_{2}(\mathbb{T})$. Then the Toeplitz operator $f \mapsto P s^{*} f$ is a contraction from $\mathbf{H}_{2}(\mathbb{T})$ into $\mathbf{H}_{2}(\mathbb{T})$. It admits thus a matrix representation. Using the basis $1, z, z^{2}, \ldots$ we see that

$$
\left\langle P s^{*} z^{n}, z^{m}\right\rangle_{2}= \begin{cases}0, & n<m \\ s_{n-m}, & n \geq m\end{cases}
$$

and hence the Toeplitz matrix representation. For the converse, we assume that $T_{s}$ is a contraction. We compute $T_{s}^{*} e_{z}$ where $e_{z}=\left(1, z, z^{2}, \ldots\right)^{t}$. We have [compare with (2.35)]

$$
T_{s}^{*} e_{z}=\overline{s(z)} e_{z}
$$

and hence the result.

\section{Positive Operators, Reproducing Kernel Spaces and Toeplitz Operators}

We use various tools from quaternionic operator theory and in particular from the theory of linear relations and the theory of reproducing kernel spaces, as developed in [8]. We recall:

Definition 3.1. Given two right (or left, or two-sided) linear spaces $\mathcal{V}, \mathcal{W}$ over the quaternions, a linear relation is a linear subspace of the Cartesian product $\mathcal{V} \times \mathcal{W}$.

The graph of a (possibly not everywhere defined) linear operator is a linear relation, but there are linear relations which are not graphs of operators. 
We will define inner products on a quaternionic right vector space, say $\mathcal{V}$, with the following convention

$$
\langle f u, g v\rangle=\bar{v}\langle f, g\rangle u, \quad f, g \in \mathcal{V}, \quad u, v \in \mathbb{H}
$$

and satisfying moreover

$$
\langle f, u g\rangle=\langle\bar{u} f, g\rangle, \quad f, g \in \mathcal{V}, u \in \mathbb{H},
$$

when the quaternionic space under study is two-sided (for instance, $\ell_{2}\left(\mathbb{N}_{0}, \mathbb{H}\right)$ ).

Let $K(x, y)$ be a the $\mathbb{H}^{r \times r}$-valued function, positive definite on $\Omega$. We will denote by $\mathcal{H}(K)$ the reproducing kernel space of $\mathbb{H}^{r}$-valued functions with reproducing kernel $K$.

Let $K_{1}(z, w)$ and $K_{2}(z, w)$ be two $\mathbb{H}^{r \times r}$-valued functions positive definite on a set $\Omega$. We recall that $K_{1} \leq K_{2}$ means that the difference $K_{2}-K_{1}$ is still positive definite in $\Omega$. This happens if and only if the space $\mathcal{H}\left(K_{1}\right)$ is contractively included in the space $\mathcal{H}\left(K_{2}\right)$.

The following result, relating operator ranges and reproducing kernel Hilbert spaces is well known. See [9] for a discussion in the quaternionic and indefinite inner product setting.

Proposition 3.2. Let $\Gamma$ be a positive bounded operator from the left quaternionic Hilbert space $\mathcal{H}$ into itself. Let $\Omega$ be a set and let $z \mapsto f_{z}$ be a $\mathcal{H}$-valued function defined on $\Omega$, and such that the closed left-linear span of the vectors $f_{w}$ is equal to $\mathcal{H}$. The function

$$
K(z, w)=\left\langle\Gamma f_{w}, f_{z}\right\rangle
$$

is positive definite on $\Omega$ and the associated reproducing kernel Hilbert space with reproducing kernel consists of the functions of the form

$$
F(z)=\left\langle\sqrt{\Gamma} f, f_{z}\right\rangle, \quad f \in \mathcal{H},
$$

with norm

$$
\|F\|=\|(I-\pi) f\|
$$

where $\pi$ is the orthogonal projection onto the kernel of $\Gamma$.

Let us set $\mathcal{H}=\ell_{2}\left(\mathbb{N}_{0}, \mathbb{H}^{r}\right)$ in the previous proposition. Since $\Gamma$ is bounded, it has a block matrix representation $\Gamma=\left(\Gamma_{n m}\right)$, where $\Gamma_{m n} \in \mathbb{H}^{r \times r}$. We can write

$$
\langle\Gamma f, g\rangle=\sum_{n, m=0}^{\infty} g_{n}^{*} \Gamma_{n m} f_{m}
$$

and

$$
K(z, w)=\sum_{n, m=0}^{\infty} f_{n}(z)^{*} \Gamma_{n m} f_{m}(w) .
$$

Cases of interest in the present work are:

$$
\Omega \subset \mathbb{R}^{4} \cong \mathbb{H}, \quad \text { and denoting } z=p, \quad f_{n}(p)=\bar{p}^{n} I_{r}
$$

and

$$
\Omega \subset \mathbb{R}^{4} \cong \mathbb{H}, \quad \text { and denoting } z=x, \quad f_{n}(x)=\overline{P_{n}(x)} I_{r}
$$


Assume now $\Gamma$ to be of the form

$$
\Gamma=I-T_{S} T_{S}^{*}
$$

with $T_{S}$ as in (2.36) and where $S_{i} \in \mathbb{H}^{r \times t}, i=0,1, \ldots$ In particular, the block Toeplitz operator $T_{S}$ is a contraction. The kernel becomes in the first case

$$
\sum_{n=0}^{\infty} p^{n} \bar{q}^{n} I_{r}-\sum_{n=0}^{\infty}\left(\sum_{m=n}^{\infty} p^{m} S_{m-n}\right)\left(\sum_{m=n}^{\infty} q^{m} S_{m-n}\right)^{*}
$$

and

$$
\sum_{n=0}^{\infty} P_{n}(x) \overline{P_{n}(y)} I_{r}-\sum_{n=0}^{\infty}\left(\sum_{m=n}^{\infty} P_{m}(x) S_{m-n}\right)\left(\sum_{m=n}^{\infty} P_{m}(y) S_{m-n}\right)^{*}
$$

in the second case.

We note that, with the $\star$-product (see [8]):

$$
\sum_{m=n}^{\infty} p^{m} S_{m-n}=p^{n} \star\left(\sum_{m=0}^{\infty} p^{m} S_{m}\right)
$$

and similarly, with the $C K$-product, using (2.27) in Lemma 2.8,

$$
\sum_{m=n}^{\infty} P_{m}(x) S_{m-n}=P_{n}(x) \odot\left(\sum_{m=0}^{\infty} P_{m}(x) S_{m}\right) .
$$

The functions

$$
\sigma(p)=\sum_{m=0}^{\infty} p^{m} S_{m}
$$

and

$$
S(x)=\sum_{m=0}^{\infty} P_{m}(x) S_{m}
$$

are Schur multipliers, for the slice hyperholomorphic and for the present case (called Appell-like case), respectively.

\section{The Hardy Space and Schur Multipliers}

In this section we will introduce and study the Hardy space in this framework. To start with, we denote by $\mathcal{E}$ the ellipsoid

$$
\mathcal{E}=\left\{x \in \mathbb{R}^{4}: 9 x_{0}^{2}+x_{1}^{2}+x_{2}^{2}+x_{3}^{2}<1\right\}
$$

and we prove the following:

Lemma 4.1. The function

$$
k_{\mathcal{E}}(x, y)=\sum_{m=0}^{\infty} P_{1}^{m \odot}(x) \overline{P_{1}^{m \odot}(y)}
$$

converges and is positive definite for $x, y \in \mathcal{E}$. 
Proof. For $x \in \mathcal{E}$ we have $\left|P_{1}(x)\right|<1$ and the result follows from Corollary 2.9 .

We point out that using (2.28), we get

$$
k_{\mathcal{E}}(x, y)=\sum_{m=0}^{\infty}\left(\sum_{|\nu|=m} \zeta(x)^{\nu} \mathbf{e}^{\nu} \frac{|\nu| !}{\nu !}\right) \overline{\left(\sum_{|\mu|=m} \zeta(y)^{\mu} \mathbf{e}^{\mu} \frac{|\mu| !}{\mu !}\right)} .
$$

Remark 4.2. In [22-24] a different approach was used and a similar construction yields the Drury-Arveson kernel

$$
\begin{aligned}
K(x, y) & =\sum_{m=0}^{\infty} \sum_{|\nu|=m} \frac{|\nu| !}{\nu !} \zeta(x)^{\nu} \overline{\zeta(y)^{\nu}} \\
& =\left(1-\zeta_{1}(x) \overline{\zeta_{1}(y)}-\zeta_{2}(x) \overline{\zeta_{2}(y)}-\zeta_{3}(x) \overline{\zeta_{3}(y)}\right)^{-\odot} .
\end{aligned}
$$

Note that the formula (4.2) is easier to work with than formula (4.3). We also note that

$$
k_{\mathcal{E}}\left(x_{0}, y_{0}\right)=\frac{1}{1-9 x_{0} y_{0}}, \quad x_{0}, y_{0} \in(-1 / 3,1 / 3) .
$$

Using the polynomials $Q_{n}$ one can define the kernel (see [12, Remark 5.3])

$$
K_{Q}(x, y)=\sum_{n=0}^{\infty} Q_{n}(x) \overline{Q_{n}(y)}
$$

whose restriction to the real axis is different, indeed it is

$$
K_{Q}\left(x_{0}, y_{0}\right)=\frac{1}{1-x_{0} y_{0}}, \quad x_{0}, y_{0} \in(-1,1) .
$$

Definition 4.3. The reproducing kernel Hilbert space associated with (4.2) will be called the Hardy space, and denoted by $\mathbf{H}_{2}(\mathcal{E})$.

Theorem 4.4. The Hardy space $\mathbf{H}_{2}(\mathcal{E})$ consists of functions of the form

$$
f(x)=\sum_{m=0}^{\infty}\left(\zeta_{1}(x) \mathbf{e}_{1}+\zeta_{2}(x) \mathbf{e}_{2}+\zeta_{3}(x) \mathbf{e}_{3}\right)^{m \odot} f_{m}=\sum_{m=0}^{\infty} P_{m}(x) f_{m},
$$

where the coefficients $f_{m}$ belong to $\mathbb{H}$ and are such that

$$
\sum_{m=0}^{\infty}\left|f_{m}\right|^{2}<\infty
$$

This expression is then the square of the norm of $f$ in the Hardy space, i.e. $\|f\|^{2}=\sum_{m=0}^{\infty}\left|f_{m}\right|^{2}$.

Proof. The proofs follows standard arguments, see $[12,21]$.

From the form of the elements of the Hardy space $\mathbf{H}_{2}(\mathcal{E})$ and using the fact that the polynomials $P_{m}$ are Fueter hyperholomorphic of axial type, see Remark 3.9 in [12], we deduce:

Corollary 4.5. Elements of $\mathbf{H}_{2}(\mathcal{E})$ are Fueter hyperholomorphic of axial type, in particular are uniquely determined by their restriction to $(-1 / 3,1 / 3)$. 
Lemma 4.6. The operator $\mathrm{S}: f \mapsto P_{1} \odot f$ is an isometry in the Hardy space, with adjoint given by

$$
\mathrm{S}^{*}\left(\sum_{n=0}^{\infty} P_{n} f_{n}\right)=\sum_{n=0}^{\infty} P_{n} f_{n+1} .
$$

Furthermore

$$
\mathrm{SS}^{*} f=f-f(0), \quad f \in \mathbf{H}_{2}(\mathcal{E}) .
$$

Proof. The proof is a consequence of

$$
\begin{aligned}
\text { SS }^{*} f & =P_{1} \odot\left(\sum_{n=0}^{\infty} P_{n} f_{n+1}\right) \\
& =\sum_{n=0}^{\infty} P_{n+1} f_{n+1} \\
& =f-f_{0} \\
& =f-f(0) .
\end{aligned}
$$

Let $C f=f(0)$ be the point evaluation in $\mathbf{H}_{2}(\mathcal{E})$. Then $C^{*} u=k_{\mathcal{E}}(\cdot, 0) u=$ $u$ and we get from the previous lemma

$$
I-M_{P_{1}} M_{P_{1}}^{*}=C^{*} C .
$$

This equation is really what makes the arguments work in the sequel, and in particular in the construction of a coisometric realization.

Definition 4.7. The operator (4.8) will be called the backward-shift operator and denoted by $R_{0}$.

Example 4.8. Let $a \in \mathcal{E}$. The space of functions in the Hardy space such that $f(a)=0$ need not be S-invariant. On the other hand, the space of functions $f \in \mathbf{H}_{2}(\mathcal{E})$ such that

$$
\left(P_{n} \odot f\right)(a)=0, \quad n=0,1,2, \ldots
$$

is S-invariant, see Lemma 2.8. This suggests that the natural homogeneous interpolation condition is (4.11) and not merely $f(a)=0$. See [23, p. 148] for a related remark.

We can consider hyperholomorphic functions operator-valued, in particular matrix or vector-valued. The definition of this class of functions is given by following the classical complex case, but we repeat it for the sake of completeness.

Definition 4.9. Let $\mathcal{X}$ be a two-sided quaternionic Banach space, and $\mathcal{X}^{*}$ be its dual. A function $f: U \subset \mathbb{H} \rightarrow \mathcal{X}$, where $U$ is open, is said to be weakly (left) hyperholomorphic in $U$ if $\Lambda f$ satisfies $D(\Lambda f)=0$ for every $\Lambda \in \mathcal{X}^{*}$. 
We recall that a function is hyperholomorphic if and only if it is differentiable in a suitable sense, see [74, Theorem 3] and we follow this notion of differentiability to state the following definition, in which we identify $\mathbb{H}$ with $\mathbb{H}_{3}=\left\{\vec{\zeta}=\left(\zeta_{1}, \zeta_{2}, \zeta_{3}\right) \mid \zeta_{i}=x_{i}-\mathbf{e}_{i} x_{0}, i=1,2,3\right\}$ as a real linear space via the $\operatorname{map}\left(\zeta_{1}, \zeta_{2}, \zeta_{3}\right) \mapsto \zeta_{1} \mathbf{e}_{1}+\zeta_{2} \mathbf{e}_{2}+\zeta_{3} \mathbf{e}_{3}$ :

Definition 4.10. Let $\vec{a} \in \mathbb{H}_{3}, U$ be a neighborhood of $\vec{a}$ and let $F: U \rightarrow \mathcal{X}$ be a continuous function. Then $f$ is called left (resp. right) strongly differentiable in $\vec{a}$ in the quaternionic sense if there exists a left (resp. right) linear map $L: \mathbb{H}_{3} \rightarrow \mathcal{X}$ such that

$$
\lim _{\Delta \vec{z} \rightarrow 0} \frac{\|f(a+\overrightarrow{+} \vec{z})-f(\vec{a})-L(\Delta \vec{z})\|_{\mathcal{X}}}{\|\Delta \vec{z}\|}=0
$$

where $\|\vec{z}\|=\sum_{i=1}^{3} \bar{\zeta}_{i} \zeta_{i}$. A function is strongly differentiable in $U$ if it is so at every point $\vec{a} \in U$.

The definition originally considered by Malonek in [74] can be obtained from the previous one when $\mathcal{X}=\mathbb{H}$. Since, in the scalar case, the definition is also equivalent to that one of left (resp. right) hyperholomorphy, we will equivalently say that a function $f$ as in Definition 4.12 is strongly hyperholomorphic. See also [19] for a theory of hyperholomorphic functions whose values are taken in a Banach algebra. Using the same arguments as in the complex case, see [77, Theorem VI.4], which are valid also in the quaternionic case, see [8], one can prove:

Theorem 4.11. A function is weakly hyperholomorphic in $U$ if and only if it is strongly hyperholomorphic in $U$.

The validity of this result allows to simplify the terminology and we shall say, for short, that $f$ is hyperholomorphic with values in $\mathcal{X}$. In the special case in which $\mathcal{X}=\mathbb{H}^{r \times s}$, a function is weakly hyperholomorphic if and only if all its entries are left or right hyperholomorphic there.

The next result was proved in the quaternionic setting in the context of slice hyperholomorphic functions, see e.g. [8, Sect. 7]; here we prove its counterpart in the present framework.

Theorem 4.12. Let $\mathfrak{M}$ be a finite dimensional linear right-vector space of $\mathbb{H}^{r}$ valued functions, and hyperholomorphic of axial type in a neighborhood of the origin. Then $\mathfrak{M}$ is $R_{0}$-invariant if and only if there exists a pair of matrices $(\mathrm{C}, \mathrm{A}) \in \mathbb{H}^{r \times N} \times \mathbb{H}^{N \times N}$ such that $f \in \mathfrak{M}$ if and only if it can be written as

$$
f=\sum_{n=0}^{\infty} P_{n} \mathrm{CA}^{n} \xi, \quad \xi \in \mathbb{H}^{N} .
$$

We have $N \geq \operatorname{dim} \mathfrak{M}$, and there is equality if and only if the pair $(C, A)$ is observable, meaning

$$
\cap_{n=0}^{\infty} \operatorname{ker} \mathrm{CA}^{n}=\{0\} .
$$


Proof. Let $f_{1}, \ldots, f_{N}$ be a basis of $\mathfrak{M}$, and let $F=\left(f_{1} f_{2} \cdots f_{N}\right)$. Let $F=$ $\sum_{n=0}^{\infty} P_{n} F_{n}$, with $F_{n} \in \mathbb{H}^{r \times N}$. In view of the $R_{0}$-invariance there exists a matrix $\mathrm{A} \in \mathbb{H}^{N \times N}$ such that

$$
\sum_{n=0}^{\infty} P_{n} F_{n+1}=\left(\sum_{n=0}^{\infty} P_{n} F_{n}\right) \text { A. }
$$

It follows that

$$
F_{n+1}=F_{n} \mathrm{~A}, \quad n=0,1, \ldots
$$

and (4.13) follows with $F_{0}=$ C.

The last claim follows from the fact that,

$$
f \equiv 0 \Longleftrightarrow \mathrm{CA}^{n} \xi=0, \quad n=0,1,2, \ldots
$$

Note that, for $x_{1}=x_{2}=x_{3}=0$, we have

$$
f\left(x_{0}\right)=\mathrm{C}\left(I_{N}-3 x_{0} \mathrm{~A}\right)^{-1} \xi .
$$

Since the $C K$-product is not a law of composition we cannot, a priori, define Schur multipliers (see Definition 4.13) in terms of multiplication operators. We define them in terms of positive definite functions. The corresponding contractive operator, counterpart of the $C K$-multiplication by $S$, is given in Proposition 4.17 and Definition 4.20; see Eq. (4.23).

Definition 4.13. The $\mathbb{H}^{r \times s}$-valued hyperholomorphic function $S$ is called a Schur multiplier if the kernel

$$
K_{S}(x, y)=\sum_{n=0}^{\infty}\left(P_{n}(x) \overline{P_{n}(y)} I_{r}-\left(P_{n} \odot S\right)(x)\left(\left(P_{n} \odot S\right)(y)\right)^{*}\right)
$$

is positive definite in $\left\{x \in \mathbb{R}^{4}: 9 x_{0}^{2}+x_{1}^{2}+x_{2}^{2}+x_{3}^{2}<1\right\}$.

Example 4.14. For instance $S=P_{1} I_{r}$ is a Schur multiplier since

$$
\begin{aligned}
& \sum_{n=0}^{\infty}\left(P_{n}(x) \overline{P_{n}(y)} I_{r}-\left(P_{n} \odot S\right)(x)\left(\left(P_{n} \odot S\right)(y)\right)^{*}\right) \\
& =\sum_{n=0}^{\infty}\left(P_{n}(x) \overline{P_{n}(y)} I_{r}-\left(P_{n} \odot P_{1}\right)(x) \overline{\left(P_{n} \odot P_{1}\right)(y)} I_{r}\right) \\
& =\sum_{n=0}^{\infty} P_{n}(x) \overline{P_{n}(y)} I_{r}-\sum_{n=0}^{\infty} P_{n+1}(x) \overline{P_{n+1}(y)} I_{r} \\
& =I_{r} .
\end{aligned}
$$

This example is of course quite trivial. We will give in Sect. 8 a complete characterization of Schur multipliers, from which one can get numerous other examples.

The positivity of the kernel (4.15) is equivalent to the contractive inclusion of the reproducing kernel Hilbert space with reproducing kernel

$$
\sum_{n=0}^{\infty}\left(P_{n} \odot S\right)(x)\left(\left(P_{n} \odot S\right)(y)\right)^{*}
$$


inside the Hardy space. In particular, if $S$ is a Schur multiplier $P_{n} \odot S \in \mathbf{H}_{2}(\mathcal{E})$ for every $n$.

Theorem 4.15. The $\mathbb{H}^{r \times s}$-valued function $S$ is a Schur multiplier if and only if the lower triangular Toeplitz operator

$$
\mathscr{T}=\left(\begin{array}{cccc}
S_{0} & 0 & 0 & \cdots \\
S_{1} & S_{0} & 0 & \cdots \\
S_{2} & S_{1} & S_{0} & 0 \\
\vdots & \ddots & \ddots & \ddots
\end{array}\right)
$$

is a contraction from $\ell_{2}\left(\mathbb{N}_{0}, \mathbb{H}^{s}\right)$ into $\ell_{2}\left(\mathbb{N}_{0}, \mathbb{H}^{r}\right)$.

Proof. Assume first that the kernel (4.15) is positive definite in $\mathcal{E}$. We divide the argument in a number of steps.

STEP 1: There exist coefficients $S_{0}, S_{1}, \ldots \in \mathbb{H}^{r \times s}$ such that

$$
S(x)=\sum_{u=0}^{\infty} P_{u}(x) S_{u}
$$

and $\sum_{u=0}^{\infty}\left\|S_{u}\right\|^{2}<\infty$.

Indeed, let $K_{2}(x, y)=\sum_{n=0}^{\infty} P_{n}(x) I_{r} \overline{P_{n}(y)}$ and $K_{1}(x, y)=\sum_{n=0}^{\infty}\left(P_{n} \odot\right.$ $S)(x)((P \odot S)(y))^{*}$. The inclusion operator

$$
\mathcal{I}\left(\sum_{n=0}^{\infty}\left(P_{n} \odot S\right) f_{n}\right)=\sum_{n=0}^{\infty}\left(P_{n} \odot S\right) f_{n}
$$

is a contraction from $\mathcal{H}\left(K_{1}\right)$ inside $\mathcal{H}\left(K_{2}\right)$, and so in particular $S \in\left(\mathbf{H}_{2}(\mathcal{E})\right)^{r \times s}$.

STEP 2: The function

$$
s(a)=\sum_{n=0}^{\infty} \chi\left(S_{n}\right) a^{n}, \quad a \in(-1,1),
$$

has an analytic contractive extension to the open unit disk of the complex plane.

We write for $x_{0}, y_{0} \in(-1 / 3,1 / 3)$

$$
K_{s}\left(3 x_{0}, 3 y_{0}\right)=\frac{I_{r}-s\left(3 x_{0}\right) s\left(3 y_{0}\right)^{*}}{1-9 x_{0} y_{0}}
$$

(of course, $K_{s}\left(3 x_{0}, 3 y_{0}\right)$ does not characterize in a unique way $K_{s}(x, y)$ ). The kernel $K_{s}\left(3 x_{0}, 3 y_{0}\right)$ is positive definite for $x_{0}, y_{0} \in(-1 / 3,1 / 3)$. We set $a=3 x_{0}$ and $b=3 y_{0}$. The kernel

$$
\frac{I_{r}-s(a) s(b)^{*}}{1-a b}
$$

is positive definite in $(-1,1)$, and so is the kernel $\chi\left(\frac{I_{r}-s(a) s(b)^{*}}{1-a b}\right)$, and we conclude by applying Theorem 2.21 .

STEP 3: The Toeplitz operator based on the sequence $\chi\left(S_{u}\right)$ is contractive from $\ell_{2}\left(\mathbb{N}_{0}, \mathbb{C}^{2 s}\right)$ into $\ell_{2}\left(\mathbb{N}_{0}, \mathbb{C}^{2}\right)$

This follows from Theorem 2.22. 
STEP 4: The Toeplitz operator $T_{s}$ is contractive.

This follows from Proposition 2.2. We restrict the operator in Step 3 to sequences of matrices in the range of $\chi$.

We now suppose that $\mathscr{T}$ is a contractive operator. We write

$$
\left\langle\left(I-\mathscr{T} \mathscr{T}^{*}\right) c, c\right\rangle_{\ell_{2}\left(\mathbb{N}_{0}, \mathbb{H}^{r}\right)} \geq 0
$$

with

$$
c=\sum_{i}\left(\begin{array}{c}
I_{r} \\
\overline{P_{1}\left(x^{(i)}\right)} I_{r} \\
\overline{P_{2}\left(x^{(i)}\right)} I_{r} \\
\vdots
\end{array}\right) u_{i}
$$

to get the positivity of the kernel $K_{S}$.

Corollary 4.16. In the above notation, the function

$$
\sum_{n=0}^{\infty} p^{n} S_{n}
$$

is a slice hyperholomorphic Schur multiplier.

Given two multipliers, the bounded operator $M_{S_{1}} M_{S_{2}}$ will not be a multiplier in general.

Proposition 4.17. Let $S$ be a $\mathbb{H}^{r \times s}$-valued Schur multiplier. The formula

$$
T\left(\sum_{n=0}^{\infty} P_{n} \overline{P_{n}(a)} u\right)=\sum_{n=0}^{\infty} P_{n}\left(\left(P_{n} \odot S\right)(a)\right)^{*} u, \quad a \in \mathcal{E}, u \in \mathbb{H}^{r},
$$

defines a contraction from $\left(\mathbf{H}_{2}(\mathcal{E})\right)^{s}$ into $\left(\mathbf{H}_{2}(\mathcal{E})\right)^{r}$, with adjoint given by

$$
T^{*}\left(\sum_{n=0}^{\infty} P_{n} u_{n}\right)=\sum_{n=0}^{\infty}\left(P_{n} \odot S\right) u_{n}, \quad u_{n} \in \mathbb{H}^{s} .
$$

Proof. Let $\sum_{n=0}^{\infty} P_{n} u_{n} \in \mathbf{H}_{2}(\mathcal{E})$. We can write:

$$
\begin{aligned}
\left\langle T^{*}\left(\sum_{n=0}^{\infty} P_{n} u_{n}\right), \sum_{n=0}^{\infty} P_{n} \overline{P_{n}(b)} u\right\rangle & =\left\langle\sum_{n=0}^{\infty} P_{n} u_{n}, T\left(\sum_{n=0}^{\infty} P_{n} \overline{P_{n}(b)} u\right)\right\rangle \\
& =\left\langle\sum_{n=0}^{\infty} P_{n} u_{n}, \sum_{n=0}^{\infty} P_{n}\left(\left(P_{n} \odot S\right)(b)\right)^{*} u\right\rangle \\
& =u^{*} \sum_{n=0}^{\infty}\left(P_{n} \odot S\right)(b) u_{n} .
\end{aligned}
$$


Remark 4.18. Formula (4.21) gives the adjoint of $C K$-multiplication for any (bounded) multiplier (i.e. functions for which the corresponding operator of $C K$-multiplication is bounded in the Hardy space), and not only for Schur multipliers.

Corollary 4.19. Let $S(x)=\sum_{n=0}^{\infty} P_{n} S_{n}$ be a $\mathbb{H}^{r \times t}$-valued Schur multiplier. Then,

$$
\widetilde{S}(x)=\sum_{n=0}^{\infty} P_{n}(x) S_{n}^{*}
$$

is a $\mathbb{H}^{t \times r}$-valued Schur multiplier.

Proof. $S$ is a Schur multiplier if and only if the function $\sum_{n=0}^{\infty} z^{n} \chi\left(S_{n}\right)$ is analytic and contractive in $\mathbb{D}$; this will hold if and only if the function

$$
\sum_{n=0}^{\infty} z^{n} \chi\left(S_{n}\right)^{*}=\sum_{n=0}^{\infty} z^{n} \chi\left(S_{n}^{*}\right)
$$

is analytic and contractive in $\mathbb{D}$; by the previous theorem this will hold if and only if $\widetilde{S}$ is a $\mathbb{H}^{t \times r}$-valued Schur multiplier.

The operator $T^{*}$ cannot be written as $S \odot\left(\sum_{n=0}^{\infty} P_{n} u_{n}\right)$, i.e. it is not the $\odot$ multiplication by $S$. We now introduce the counterpart of this latter operator here.

Definition 4.20. The operator $T^{*}$ will be denoted by $M_{S}$ :

$$
M_{S}\left(\sum_{n=0}^{\infty} P_{n}(a) u_{n}\right)=\sum_{n=0}^{\infty}\left(P_{n} \odot S\right)(a) u_{n}, \quad a \in \mathcal{E} .
$$

We have the following extension result, counterpart of Theorem 2.21. The proof is slightly different.

Theorem 4.21. Assume the kernel (4.15) defined and positive definite in a neighborhood $N$ of the origin of $\mathbb{R}^{4}$. Then, $S$ extends, in a unique way, to a Schur multiplier.

Proof. We consider the scalar case to simplify the notation. The preceding argument still holds and, setting $a=0$ and $b \in N$, we get

$$
\left(T^{*} v\right)(b)=S(b) v, \quad b \in N .
$$

But $T^{*} v \in \mathbf{H}_{2}(\mathcal{E})$ and in particular is hyperholomorphic in all of $\mathcal{E}$. More generally, still for $b \in N$, but for $f=\sum_{n=0}^{\infty} P_{n} f_{n} \in \mathbf{H}_{2}(\mathcal{E})$ we have

$$
\begin{aligned}
\left(T^{*} f\right)(b) & =\left\langle\left(T^{*} f\right)(\cdot), k_{\mathcal{E}}(\cdot, b)\right\rangle_{2} \\
& =\left\langle f(\cdot), T k_{\mathcal{E}}(\cdot, v)\right\rangle_{2} \\
& =\sum_{n=0}^{\infty}\left(P_{n} \odot S\right)(b) f_{n} .
\end{aligned}
$$


Writing $\left(T^{*} f\right)(b)=\sum_{n=0}^{\infty} P_{n} h_{n}$ and $S=\sum_{n=0}^{\infty} P_{n} s_{n}$, the previous equality is equivalent (since $v$ varies in an open set; it would be enough to have an interval such that $\left.x_{0} \in(-\epsilon, \epsilon)\right)$

$$
\left(\begin{array}{c}
h_{0} \\
h_{1} \\
\vdots
\end{array}\right)=T_{S}\left(\begin{array}{c}
f_{0} \\
f_{1} \\
\vdots
\end{array}\right),
$$

where $T_{S}$ is the lower triangular Toeplitz operator based on the coefficients of $S$. So $T_{S}$ is a contraction, and so $I-T_{S} T_{S}^{*} \geq 0$, and so $\left\langle\left(I-T_{S} T_{S}^{*} c, c\right\rangle \geq 0\right.$ for every $c \in \ell_{2}\left(\mathbb{N}_{0}, \mathbb{H}\right)$. The choice (4.18) allows to conclude that $S$ is a Schur multiplier.

Lemma 4.22. The following equality holds for all $f \in \mathbf{H}_{2}(\mathcal{E})$ :

$$
P_{1} \odot\left(M_{S} f\right)=M_{S}\left(P_{1} \odot f\right) .
$$

Proof. Let $f=\sum_{n=0}^{\infty} P_{n} u_{n}$. We can write:

$$
\begin{aligned}
P_{1} \odot\left(M_{S} f\right) & =P_{1} \odot\left(\sum_{n=0}^{\infty}\left(P_{n} \odot S\right) u_{n}\right) \\
& =\sum_{n=0}^{\infty}\left(P_{1+n} \odot S\right) u_{n} \\
& =M_{S}\left(\sum_{n=0}^{\infty} P_{n+1} u_{n}\right) \\
& =M_{S}\left(P_{1} \odot f\right) \\
& =M_{S}\left(M_{P_{1}} f\right) .
\end{aligned}
$$

It is useful to rewrite (4.24) as

$$
M_{P_{1}} M_{S}=M_{S} M_{P_{1}} \text {. }
$$

\section{Schur Algorithm}

The Schur algorithm is based on Schwarz lemma and on the fact that if two numbers $u$ and $v$ are in the open unit disk so is $\frac{u-v}{1-u \bar{v}}$. It reads (see $[80,81]$ ):

Theorem 5.1. Let $f$ be analytic and contractive in the open unit disk (i.e. a Schur function), and assume $|f(0)|<1$, and set $f^{(0)}=f$. Then the recursion

$$
f^{(n+1)}(z)= \begin{cases}\frac{f^{(n)}(z)-f^{(n)}(0)}{z\left(1-\overline{f^{(n)}(0)} f^{(n)}(z)\right)}, & 0<|z|<1, \\ \frac{\left(f^{(n)}\right)^{\prime}(0)}{1-\left|f^{(n)}(0)\right|^{2}}, & z=0,\end{cases}
$$

defines a family of Schur functions; it stops at rank $n_{0}$ if $\left|f^{\left(n_{0}\right)}(0)\right|=1$. 
The numbers $\rho_{n}=f^{(n)}(0)$ are called the Schur parameters associated with the Schur function $f$.

This recursion cannot be considered directly in the matrix-valued case. One needs to take into account that if $E_{1}$ and $E_{2}$ are strictly contractive matrices, say in $\mathbb{C}^{p \times q}$, the matrix $\left(E_{1}+E_{2}\right)\left(I_{q}+E_{1}^{*} E_{2}\right)^{-1}$ need not be contractive, but the matrix

$$
\left(I_{p}-E_{1} E_{1}^{*}\right)^{1 / 2}\left(E_{2}+E_{1}\right)\left(I_{p}+E_{1}^{*} E_{2}\right)^{-1}\left(I_{q}-E_{1}^{*} E_{1}\right)^{1 / 2}
$$

is strictly contractive.

The matricial Schur algorithm was studied in [48] and, in the next result, we repeat the statement taking into account the matrix symmetry

$$
E_{p}^{-1} \bar{M} E_{q}=M, \quad E_{m}=\left(\begin{array}{cc}
0 & I_{m} \\
-I_{m} & 0
\end{array}\right), m=p, q
$$

is in force.

Theorem 5.2. Let $s$ be a $\mathbb{C}^{p \times q}$-valued Schur function satisfying (5.3). Assume $s_{0}$ strictly contractive. Then the function

$$
s^{(1)}(z)=\left\{\begin{array}{l}
\frac{1}{z}\left(I_{p}-s_{0} s_{0}^{*}\right)^{-1 / 2}\left(s-s_{0}\right)\left(I_{q}-s_{0}^{*} s\right)^{-1}\left(I_{q}-s_{0}^{*} s_{0}\right)^{1 / 2}, \quad 0<|z|<1, \\
\left(I_{p}-s_{0} s_{0}^{*}\right)^{-1 / 2} s^{\prime}(0)\left(I_{q}-s_{0}^{*} s_{0}\right)^{-1 / 2}, \quad z=0,
\end{array}\right.
$$

is a Schur function and satisfies the symmetry (5.3).

If $\left\|s_{0}\right\|<1$ one can iterate, and one gets the matricial Schur algorithm. The condition $\|s(0)\|<1$ is quite restrictive. A tangential Schur algorithm was developed in [16]. On the other hand, when $p=q=2$ and $s(0)$ is in the range of $\chi$ both $\left(I_{2}-s_{0} s_{0}^{*}\right)^{-1 / 2}$ and $\left(I_{2}-s_{0}^{*} s_{0}\right)^{1 / 2}$ are scalar matrices and (5.4) reduces to

$$
s^{(1)}(z)=\frac{1}{z}\left(s(z)-s_{0}\right)\left(I_{2}-s_{0}^{*} s(z)\right)^{-1} .
$$

We now turn to the setting of hyperholomorphic functions of axial type. For simplicity of exposition we first consider scalar valued Schur multiplier. From the analysis in the previous sections, $S=\sum_{n=0}^{\infty} P_{n} S_{n}$ is a Schur multiplier if and only if the block Toeplitz operator

$$
\left(\begin{array}{cccc}
\chi\left(S_{0}\right) & 0 & 0 & \ldots \\
\chi\left(S_{1}\right) & \chi\left(S_{0}\right) & 0 & \ldots \\
\chi\left(S_{2}\right) & \chi\left(S_{1}\right) & \chi\left(S_{0}\right) & 0 \\
\cdots & & \ldots
\end{array}\right)
$$

is a contraction from $\ell_{2}\left(\mathbb{N}_{0}, \mathbb{C}^{2}\right)$ into $\ell_{2}\left(\mathbb{N}_{0}, \mathbb{C}^{2}\right)$. The function

$$
s(z)=\sum_{n=0}^{\infty} \chi\left(S_{n}\right) z^{n}
$$

takes then contractive values, and is in the range of $\chi$; see Lemma 2.1. 
Theorem 5.3. Let $S$ be a quaternionic Schur multiplier such that $|S(0)|<1$. Then the function

$$
S^{(1)}\left(3 x_{0}\right)=\frac{1}{3 x_{0}}\left(S\left(3 x_{0}\right)-S(0)\right)\left(1-\overline{S(0)} S\left(3 x_{0}\right)\right)^{-1}
$$

extends to a Schur multiplier.

In the matrix-valued case it is not true anymore that $I-\chi\left(S_{0}\right) \chi\left(S_{0}\right)^{*}$ is a scalar matrix.

Theorem 5.4. Let $S$ be a $\mathbb{H}^{r \times t}$-valued Schur multiplier, and assume $\|S(0)\|<$ 1. The function

$$
S^{(1)}\left(3 x_{0}\right)=\frac{1}{3 x_{0}}\left(I_{r}-S_{0} S_{0}^{*}\right)^{-1 / 2}\left(S\left(3 x_{0}\right)-S_{0}\right)\left(I_{t}-S_{0}^{*} S\left(3 x_{0}\right)\right)^{-1}\left(I_{t}-S_{0}^{*} S_{0}\right)^{1 / 2},
$$

with $x_{0} \in(-1 / 3,1 / 3)$ and $S_{0}=S(0)$ extends to a Schur multiplier.

The question whether the tangential Schur algorithm developed in [16] can lead to functions satisfying the required symmetry property in the matrixvalued case remains to be considered.

\section{Intrinsic Functions}

In this section we study quaternionic intrinsic Fueter hyperholomorphic functions. Let us recall that, given an hyperholomorphic function $f$ on some axially symmetric open set $\Omega$, we say that $f$ is quaternionic intrinsic if it satisfies the relation

$$
f(\bar{x})=\overline{f(x)}, \forall x \in \Omega .
$$

Proposition 6.1. The family of polynomials $\left(P_{n}\right)_{n \geq 0}$ consists of axially hyperholomorphic quaternionic intrinsic functions on $\mathbb{H}$.

Proof. We know that for all $n \geq 0$ the polynomials $P_{n}$ are axially hyperholomorphic functions on $\mathbb{H}$. Furthermore, using the relation with the $n$-th quaternionic Appell polynomials $Q_{n}$, see [12, (3.8)], we have

$$
\begin{aligned}
\overline{P_{n}(x)} & =\frac{\overline{Q_{n}(x)}}{c_{n}} \\
& =\sum_{j=0}^{n} \frac{T_{j}^{n}}{c_{n}} \bar{x}^{n-j} x^{j} \\
& =\frac{Q_{n}(\bar{x})}{c_{n}} \\
& =P_{n}(\bar{x}) .
\end{aligned}
$$

Proposition 6.2. Let $f$ be a hyperholomorphic function of axial type on some axially symmetric open set $\Omega$. Then, $f$ is quaternionic intrinsic if and only if it admits a power series representation with real coefficients with respect to the polynomials $\left(P_{n}\right)_{n \geq 0}$. 
Proof. We know by Theorem 3.10 in [12] that $f$ admits a power series with respect to $\left(P_{n}\right)_{n \geq 0}$. So, we can write $f=\sum_{n=0}^{\infty} P_{n} f_{n}$ with $f_{n} \in \mathbb{H}$ for all $n \geq 0$. We assume that $f$ is intrinsic, thus the formula (6.1) and Proposition 6.1 imply that

$$
\begin{aligned}
\overline{f(x)}=f(\bar{x}), \forall x \in \Omega & \Leftrightarrow \sum_{n=0}^{\infty} \overline{P_{n}(x) f_{n}}=\sum_{n=0}^{\infty} P_{n}(\bar{x}) f_{n}, \forall x \in \Omega \\
& \Leftrightarrow \sum_{n=0}^{\infty} \overline{f_{n}} \overline{P_{n}(x)}=\sum_{n=0}^{\infty} \overline{P_{n}(x)} f_{n}, \forall x \in \Omega \\
& \Leftrightarrow \sum_{n=0}^{\infty} \overline{f_{n}}\left(3 x_{0}\right)^{n}=\sum_{n=0}^{\infty}\left(3 x_{0}\right)^{n} f_{n}, \forall x_{0} \in \mathbb{R} \\
& \Leftrightarrow \overline{f_{n}}=f_{n}, \forall n \geq 0 \\
& \Leftrightarrow f_{n} \in \mathbb{R}, \forall n \geq 0 .
\end{aligned}
$$

The equivalence between the second and the third lines holds because $P_{n}$ is the unique axially hyperholomorphic extension of $\left(3 x_{0}\right)^{n}$. This ends the proof.

Proposition 6.3. Let $S_{1}$ and $S_{2}$ be two hyperholomorphic functions of axial type, defined on some axially symmetric open set $\Omega$. If $S_{1}$ is quaternionic intrinsic, then $S_{1} \odot S_{2}$ admits a power series expansion with respect to the polynomials $\left(P_{n}\right)_{n \geq 0}$.

Proof. We note that $S_{1}$ and $S_{2}$ have power series expansions in terms of $\left(P_{n}\right)_{n \geq 0}$ that we can write $S_{1}=\sum_{n=0}^{\infty} P_{n} a_{n}$ and $S_{2}=\sum_{n=0}^{\infty} P_{n} b_{n}$. Since $S_{1}$ is quaternionic intrinsic we know by Proposition 6.2 that the coefficients $\left(a_{n}\right)_{n \geq 0}$ are real. Thus, we apply also Lemma 2.8 to get

$$
\begin{aligned}
S_{1} \odot S_{2} & =\left(\sum_{n=0}^{\infty} P_{n} a_{n}\right) \odot\left(\sum_{m=0}^{\infty} P_{m} b_{m}\right) \\
& =\sum_{n, m=0}^{\infty}\left(P_{n} \odot P_{m}\right) a_{n} b_{m} \\
& =\sum_{n, m=0}^{\infty} P_{n+m} a_{n} b_{m} \\
& =\sum_{n=0}^{\infty} P_{n}\left(\sum_{k=0}^{n} a_{k} b_{n-k}\right) .
\end{aligned}
$$

Proposition 6.4. Let $S$ be a hyperholomorphic function of axial type. If $S$ is quaternionic intrinsic, then the operator $M_{S}$ coincides with the multiplication operator $f \mapsto S \odot f$. 
Proof. We note that since $S$ is quaternionic intrinsic, it has real coefficients. Thus, we have $P_{n} \odot S=S \odot P_{n}$ for all $n \geq 0$. Then, starting from Definition 4.20 , for any $f=\sum_{n=0}^{\infty} P_{n} u_{n}$, we have

$$
\begin{aligned}
M_{S}(f) & =\sum_{n=0}^{\infty}\left(P_{n} \odot S\right) u_{n} \\
& =\sum_{n=0}^{\infty}\left(S \odot P_{n}\right) u_{n} \\
& =S \odot\left(\sum_{n=0}^{\infty} P_{n} u_{n}\right) \\
& =S \odot f .
\end{aligned}
$$

Proposition 6.5. Let $S_{1}$ and $S_{2}$ be two hyperholomorphic functions of axial type such that $S_{1}$ is quaternionic intrinsic. Then, we have

$$
M_{S_{1}} M_{S_{2}}=M_{S_{1} \odot S_{2}} .
$$

Proof. We know by Proposition 6.3 that $S_{1} \odot S_{2}$ is well defined and admits a power series expansion in terms of $\left(P_{n}\right)_{n \geq 0}$ since $S_{1}$ is intrinsic. Therefore, using Proposition 6.4, we have

$$
\begin{aligned}
M_{S_{1} \odot S_{2}}(f) & =\left(S_{1} \odot S_{2}\right) \odot f \\
& =M_{S_{1}}\left(S_{2} \odot f\right) \\
& =M_{S_{1}} M_{S_{2}}(f) .
\end{aligned}
$$

\section{7. de Branges-Rovnyak Spaces}

The reproducing kernel Hilbert space with reproducing kernel (4.15) will be called the de Branges-Rovnyak space associated with the Schur multiplier $S$ and denoted by $\mathcal{H}(S)$. The treatment using the Appell-like approach allows to prove results naturally extending the corresponding ones in the classical complex case. For example, we have the following characterization:

Theorem 7.1. We have

$$
\left(\left(I-M_{S} M_{S}^{*}\right)\left(k_{\mathcal{E}}(\cdot, a) u\right)(b)=K_{S}(b, a) u\right.
$$

and

$$
\mathcal{H}(S)=\operatorname{Ran} \sqrt{I-M_{S} M_{S}^{*}}
$$

with inner product

$$
\left\langle\sqrt{I-M_{S} M_{S}^{*}} f, \sqrt{I-M_{S} M_{S}^{*}} g\right\rangle_{\mathcal{H}(S)}=\langle(I-\pi) f, g\rangle_{2}
$$

where $\pi$ is the orthogonal projection on the kernel of $\sqrt{I-M_{S} M_{S}^{*}}$. 
Proof. The claims follow from [9].

We recall the following, valid for $f, g \in \mathbf{H}_{2}(\mathcal{E})$ (the first equality is a special case of the second one):

$$
\begin{aligned}
\langle\Gamma f, \Gamma g\rangle_{\mathcal{H}(S)} & =\langle\Gamma f, g\rangle_{2}, \\
\langle\sqrt{\Gamma} f, \Gamma g\rangle_{\mathcal{H}(S)} & =\langle\sqrt{\Gamma} f, \sqrt{\Gamma} g\rangle_{2} .
\end{aligned}
$$

Using the quaternionic version of [44] or [55, Theorem 4.1] (we do not give proofs of these since we will have more general results than the theorems below in the next section) we have the following results, for matrix-valued Schur multipliers

Theorem 7.2. Let $S$ be a $\mathbb{H}^{r \times s}$-valued Schur multiplier. An element $f$ in $\mathbf{H}_{2}(\mathcal{E})$ belongs to $\mathcal{H}(S)$ if and only if

$$
\sup _{g \in \mathbf{H}_{2}(\mathcal{E})}\left\|f+M_{S} g\right\|_{2}^{2}-\|g\|_{2}^{2}<\infty .
$$

Using this characterization we can prove the following:

Theorem 7.3. Let $S$ be a $\mathbb{H}^{r \times s}$-valued Schur multiplier. Let $R_{0}$ be defined by (4.8). Then:

$$
\left\|R_{0} f\right\|_{\mathcal{H}(S)}^{2} \leq\|f\|_{\mathcal{H}(S)}^{2}-\|f(0)\|^{2}, \quad f \in \mathcal{H}(S)
$$

Proof. Recall that S denotes the forward-shift operator, and that the latter is an isometry (see Lemma 4.6). Using (4.9) and (4.24) we can write for $f, g \in \mathbf{H}_{2}(\mathcal{E})$ :

$$
\begin{aligned}
\left\|R_{0} f+M_{S} g\right\|_{2}^{2}-\|g\|_{2}^{2}= & \left\|\mathrm{S}\left(R_{0} f+M_{S} g\right)\right\|_{2}^{2}-\|\mathrm{S} g\|_{2}^{2} \\
= & \left\|f-f(0)+M_{S}\left(P_{1} \odot g\right)\right\|_{2}^{2}-\left\|P_{1} \odot g\right\|_{2}^{2} \\
= & \left\|f+M_{S}\left(P_{1} \odot g\right)\right\|_{2}^{2}-2 \operatorname{Re}\left\langle f+M_{S}\left(P_{1} \odot g\right), f(0)\right\rangle+ \\
& +\|f(0)\|^{2}-\left\|P_{1} \odot g\right\|_{2}^{2} \\
= & \left\|f+M_{S}\left(P_{1} \odot g\right)\right\|_{2}^{2}-\left\|P_{1} \odot g\right\|_{2}^{2}-\|f(0)\|^{2},
\end{aligned}
$$

where we have used, with $g=\sum_{n=0}^{\infty} P_{n} b_{n}$,

$$
\begin{aligned}
\left\langle f+M_{S}\left(P_{1} \odot g\right), f(0)\right\rangle_{2} & =\left\langle f+P_{1} \odot M_{S} g, f(0)\right\rangle_{2} \\
& =\langle f, f(0)\rangle_{2}+\left\langle P_{1} \odot M_{S} g, f(0)\right\rangle_{2} \\
& =\|f(0)\|^{2}
\end{aligned}
$$

since $P_{1} \odot M_{S} g$ has no constant term.

Theorem 7.4. Let $R_{0}$ be defined by (4.8). Then $R_{0} S u \in \mathcal{H}(S)$ for every $u \in$ $\mathbb{H}^{s}$ and

$$
\left\|R_{0} S\right\|^{2} \leq\|u\|^{2}-\|S(0) u\|^{2}, \quad u \in \mathbb{H}^{s}
$$


Proof. We already know that $S u \in \mathbf{H}_{2}(\mathcal{E})$. We write

$$
\begin{aligned}
\left\|R_{0} S+M_{S} g\right\|_{2}^{2}-\|g\|_{2}^{2}= & \left\|\mathrm{S}\left(R_{0} S+M_{S} g\right)\right\|_{2}^{2}-\|\mathrm{S} g\|_{2}^{2} \\
= & \left\|-S(0) u+M_{S}\left(u+P_{1} \odot g\right)\right\|_{2}^{2}-\left\|P_{1} \odot g\right\|_{2}^{2} \\
= & \|S(0) u\|^{2}+\left\|M_{S}\left(u+P_{1} \odot g\right)\right\|^{2} \\
& -2 \operatorname{Re}\left\langle S(0), M_{S}\left(u+P_{1} \odot g\right)\right\rangle \\
\leq & \|S(0)\|_{2}+\left\|u+P_{1} \odot g\right\|_{2}^{2}-\left\|P_{1} \odot g\right\|_{2}^{2} \\
= & \|u\|^{2}-\|S(0) u\|^{2} .
\end{aligned}
$$

In the above, to go from the third to fourth line we used that

$$
\left\|M_{S}\left(u+P_{1} \odot g\right)\right\|_{2} \leq\left\|u+P_{1} \odot g\right\|_{2}
$$

(since $S$ is a Schur multiplier) and

$$
\left\langle S(0), M_{S}\left(u+P_{1} \odot g\right)\right\rangle=0
$$

since $M_{S}\left(u+P_{1} \odot g\right)$ has no constant term in its expansion along the $P_{n}$. Similarly we used that $\left\langle u, P_{1} \odot g\right\rangle_{2}=0$ to go from the fourth to the last line.

The operators defined in the previous theorems are part of a coisometric operator matrix. In [44] (see also [42]) it is obtained using the theory of complementation. In the next section we use a different method.

\section{The Coisometric Colligation and Blaschke Functions}

\subsection{The Lurking Isometry}

Let us denote by $\odot_{r}$ the right $C K$-product. Using (2.27) we note that (4.15) can be rewritten as

$$
K_{S}(x, y)-P_{1}(x) \odot K_{S}(x, y) \odot_{r} \overline{P_{1}(y)}=I_{r}-S(x) S(y)^{*},
$$

from which we get

$$
K_{S}(x, y)+S(x) S(y)^{*}=P_{1}(x) \odot K_{S}(x, y) \odot_{r} \overline{P_{1}(y)}+I_{r} .
$$

Write $K(x, y)=\langle f(x), f(y)\rangle_{\mathfrak{H}}$, where $\mathfrak{H}$ is a Hilbert space and $x \mapsto f(x)$ is $\mathfrak{H}$-valued function. We rewrite (8.2) as

$$
\left\langle\left(\begin{array}{c}
S(y)^{*} h \\
f(y)
\end{array}\right)\left(\begin{array}{c}
S(x)^{*} k \\
f(y)
\end{array}\right)\right\rangle_{\mathbb{H}^{r} \oplus \mathfrak{H}}=\left\langle\left(\begin{array}{c}
h \\
f(y) \odot_{\ell} \overline{P_{1}(y)} h
\end{array}\right)\left(\begin{array}{c}
k \\
f(x) \odot_{\ell} \overline{P_{1}(y) k}
\end{array}\right)\right\rangle_{\mathbb{H}^{s} \oplus \mathfrak{H}}
$$

where $h, k \in \mathbb{H}^{s}$ and $x, y \in \mathcal{E}$.

This last equality, called the lurking isometry (see [4,28]), can be the tool to get a co-isometric realization of $S$ (see [2] for an application in the quaternionic setting). We will choose a different (and closely related) avenue, namely the theory of relations, which originates with the work of Krein and Langer (the $\epsilon_{z}$ method; see e.g. [69,70]) and was developed further in [11]. We will use the lurking isometry method in Sect. 11 to characterize Carathéodory and Schur multipliers in the setting of the counterpart of Hardy space of the right half plane. 


\subsection{The Co-isometric Realization}

We use the method of isometric relations, as in [11], suitably adapted to the present setting, and follow $\left[22\right.$, Sect. 2]. We set $\Gamma=I-M_{S} M_{S}^{*}$, and define $w_{a}$ via

$$
w_{a} q=\Gamma M_{P_{1}}^{*} k_{\mathcal{E}}(\cdot, a) p \in \mathcal{H}(S), \quad q \in \mathbb{H}^{r},
$$

and introduce:

$$
\widehat{V}\left(\begin{array}{c}
w_{a} q \\
u
\end{array}\right)=\left(\begin{array}{c}
\left(K_{S}(\cdot, a)-K_{S}(\cdot, 0)\right) q+K_{S}(\cdot, 0) u \\
\left(S(a)^{*}-S(0)^{*}\right) q+S(0)^{*} u
\end{array}\right), \quad q \in \mathbb{H}^{r}, u \in \mathbb{H}^{s}
$$

Theorem 8.1. $\widehat{V}$ is isometric from its domain $\operatorname{Dom} \widehat{V} \subset \mathcal{H}(S) \oplus \mathbb{H}^{r}$ into $\mathcal{H}(S) \oplus \mathbb{H}^{s}$.

Proof. We want to check:

$$
\left\langle\widehat{V}\left(\begin{array}{c}
w_{a} q \\
u
\end{array}\right), \widehat{V}\left(\begin{array}{c}
w_{b} p \\
v
\end{array}\right)\right\rangle_{\mathcal{H}(S) \oplus \mathbb{H}^{s}}=\left\langle\left(\begin{array}{c}
w_{a} q \\
u
\end{array}\right),\left(\begin{array}{c}
w_{b} p \\
v
\end{array}\right)\right\rangle_{\mathcal{H}(S) \oplus \mathbb{H}^{r}}
$$

where $p, q \in \mathbb{H}^{r}, u, v \in \mathbb{H}^{s}$, and $a, b \in \mathcal{E}$. We divide the proof into three steps. STEP 1: Case $u=v=0$.

Then, only terms involving the directions $p$ and $q$ appear. In the following sequence of equality we use (4.25) to go from the second to the third line, and (4.10) to go from the fourth to the fifth line. We also note that

$$
\begin{aligned}
&\left\langle w_{a} q, w_{b} p\right\rangle_{\mathcal{H}(S)}=\left\langle\Gamma M_{P_{1}}^{*} k_{\mathcal{E}}(\cdot, a) q, M_{P_{1}}^{*} k_{\mathcal{E}}(\cdot, b) p\right\rangle_{2} \\
&=\left\langle M_{P_{1}}^{*} k_{\mathcal{E}}(\cdot, a) q, M_{P_{1}}^{*} k_{\mathcal{E}}(\cdot, b) p\right\rangle_{2} \\
& \quad-\left\langle M_{S}^{*} M_{P_{1}}^{*} k_{\mathcal{E}}(\cdot, a) q, M_{S}^{*} M_{P_{1}}^{*} k_{\mathcal{E}}(\cdot, b) p\right\rangle_{2} \\
&=\left\langle M_{P_{1}}^{*} k_{\mathcal{E}}(\cdot, a) q, M_{P_{1}}^{*} k_{\mathcal{E}}(\cdot, b) p\right\rangle_{2} \\
&-\left\langle M_{P_{1}}^{*} M_{S}^{*} k_{\mathcal{E}}(\cdot, a) q, M_{P_{1}}^{*} M_{S}^{*} k_{\mathcal{E}}(\cdot, b) p\right\rangle_{2} \\
&=\left\langle M_{P_{1}} M_{P_{1}}^{*} k_{\mathcal{E}}(\cdot, a) q, k_{\mathcal{E}}(\cdot, b) p\right\rangle_{2} \\
& \quad-\left\langle M_{P_{1}} M_{P_{1}}^{*} M_{S}^{*} k_{\mathcal{E}}(\cdot, a) q, M_{S}^{*} k_{\mathcal{E}}(\cdot, b) p\right\rangle_{2} \\
&=\left\langle\left(I-C^{*} C\right) k_{\mathcal{E}}(\cdot, a) q, k_{\mathcal{E}}(\cdot, b) p\right\rangle_{2} \\
& \quad-\left\langle\left(I-C^{*} C\right) M_{S}^{*} k_{\mathcal{E}}(\cdot, a) q, M_{S}^{*} k_{\mathcal{E}}(\cdot, b) p\right\rangle_{2} .
\end{aligned}
$$

To pursue we note that

$$
C\left(k_{\mathcal{E}}(\cdot, a) q\right)=q
$$

and, using the formula (4.20) for $M_{S}^{*}$

$$
C M_{S}^{*}\left(k_{\mathcal{E}}(\cdot, a) q\right)=S(a)^{*} q .
$$

Thus

$$
\begin{aligned}
\left\langle w_{a} q, w_{b} p\right\rangle_{\mathcal{H}(S)} & =p^{*} k_{\mathcal{E}}(b, a) q-p^{*} q+p^{*} S(b) S(a)^{*} q-\left\langle M_{S}^{*} k_{\mathcal{E}}(\cdot, a) q, M_{S}^{*} k_{\mathcal{E}}(\cdot, b) p\right\rangle_{2} \\
& =\left\langle\Gamma k_{\mathcal{E}}(\cdot, a) q, k_{\mathcal{E}}(\cdot, b) p\right\rangle_{2}-p^{*} q+p^{*} S(b) S(a)^{*} q \\
& =\left\langle K_{S}(\cdot, a) q, K_{S}(\cdot, b) p\right\rangle_{\mathcal{H}(S)}-q p^{*}+S(b) S(a)^{*} q .
\end{aligned}
$$


Furthermore,

$$
\begin{aligned}
& \left\langle\left(K_{S}(\cdot, a)-K_{S}(\cdot, 0)\right) q,\left(K_{S}(\cdot, b)-K_{S}(\cdot, 0)\right) p\right\rangle_{\mathcal{H}(S)} \\
& \quad+p^{*}(S(b)-S(0))\left(S(a)^{*}-S(0)^{*}\right) p \\
& =\left\langle K_{S}(\cdot, a) q, K_{S}(\cdot, b) p\right\rangle_{\mathcal{H}(S)}-p^{*} K_{S}(0, a) q-p^{*} K_{S}(b, 0)+p^{*} K_{S}(0,0) q+ \\
& \quad+p^{*}(S(b)-S(0))\left(S(a)^{*}-S(0)^{*}\right) p \\
& =\left\langle K_{S}(\cdot, a) q, K_{S}(\cdot, b) p\right\rangle_{\mathcal{H}(S)}-p^{*}\left(I-S(0) S(a)^{*}\right) q-p^{*}\left(I-S(b) S(0)^{*}\right) q+ \\
& \quad+p^{*}\left(I-S(0) S(0)^{*}\right) q+p^{*}(S(b)-S(0))\left(S(a)^{*}-S(0)^{*}\right) q \\
& =0 .
\end{aligned}
$$

STEP 2: Case $p=q=0$.

We now need to check that

$$
\left\langle K_{S}(\cdot, 0) u, K_{S}(\cdot, 0) v\right\rangle_{\mathcal{H}(S)}+v^{*} S(0) S(0)^{*} u=v^{*} u,
$$

but this is straightforward.

STEP 3: Mixed terms.

By symmetry it is enough to consider the case where $p$ and $u$ appears. We need to verify that:

$$
\left\langle K_{S}(\cdot, 0) u,\left(K_{S}(\cdot, b)-K_{S}(\cdot, 0)\right) p\right\rangle+\left\langle S(0)^{*} u,\left(S(b)^{*}-S(0)^{*}\right) p\right\rangle=0,
$$

but this is equivalent to

$$
K_{S}(b, 0)-K_{S}(0,0)-S(0) S(0)^{*}+S(b) S(0)^{*}=0,
$$

i.e.

$$
I_{r}-S(b) S(0)^{*}-\left(I_{r}-S(0) S(0)^{*}\right)-S(0) S(0)^{*}+S(b) S(0)^{*}=0,
$$

which clearly holds.

We now compute the adjoint of the above isometric operator.

We write

$$
\widehat{V}=\left(\begin{array}{ll}
\widehat{T} & \widehat{G} \\
\widehat{F} & \widehat{H}
\end{array}\right) .
$$

Theorem 8.2. $\widehat{V}$ is densely defined, extends to an everywhere defined isometry and its adjoint is given by

$$
\widehat{T}^{*}=R_{0} .
$$

Proof. By definition of the operator range inner product we have:

$$
\begin{aligned}
\left\langle\sqrt{\Gamma} f, \Gamma w_{a} p\right\rangle_{\mathcal{H}(S)} & =\left\langle\sqrt{\Gamma} f, M_{P_{1}}^{*} k_{\mathcal{E}}(\cdot, a) p\right\rangle_{2} \\
& =u^{*}\left(M_{P_{1}} \odot \sqrt{\Gamma} f\right)(a)
\end{aligned}
$$

and so $f=0$ if the above vanishes for all $u$ and $a$ since $M_{P_{1}}$ is an isometry.

Set $\widehat{T}^{*}(\sqrt{\Gamma} f)=\sqrt{\Gamma} g$. We have on the one hand

$$
\begin{aligned}
\left\langle\widehat{T}^{*} F, \Gamma\left(M_{P_{1}}^{*} k_{\mathcal{E}}(\cdot, a) u\right)\right\rangle_{\mathcal{H}(S)} & =\left\langle\sqrt{\Gamma} g, \Gamma\left(M_{P_{1}}^{*} k_{\mathcal{E}}(\cdot, a) u\right)\right\rangle_{\mathcal{H}(S)} \\
& =\left\langle\sqrt{\Gamma} g, M_{P_{1}}^{*} k_{\mathcal{E}}(\cdot, a) u\right\rangle_{2} \\
& =u^{*}\left(P_{1} \odot \sqrt{\Gamma} g\right)(a) .
\end{aligned}
$$


On the other hand,

$$
\begin{aligned}
\left\langle\widehat{T}^{*} F, \Gamma\left(M_{P_{1}}^{*} k_{\mathcal{E}}(\cdot, a) u\right)\right\rangle_{\mathcal{H}(S)} & =\left\langle\sqrt{\Gamma} g,\left(K_{S}(\cdot, a)-K(S(\cdot, 0)) u\right\rangle_{\mathcal{H}(S)}\right. \\
& \left.\left.=u^{*}(\sqrt{\Gamma} g)(a)-\sqrt{\Gamma} g\right)(0)\right)
\end{aligned}
$$

Hence

$$
\left(P_{1} \odot \widehat{T}^{*}(F)\right)(b)=F(b)-F(0) .
$$

Proposition 8.3. Let

$$
\widehat{V}^{*}=\left(\begin{array}{ll}
A & B \\
C & D
\end{array}\right),
$$

where $V$ is as in (8.4). Then, for $f=\sum_{n=0}^{\infty} P_{n} f_{n} \in \mathcal{H}(S)$

$$
f_{n}=C A^{n} f, \quad n=0,1, \ldots
$$

and

$$
S(x)=D+\sum_{n=1}^{\infty} P_{n}(x) C A^{n-1} B .
$$

Proof. Let $f(x)=\sum_{n=0}^{\infty} P_{n}(x) f_{n} \in \mathcal{H}(S)$. We have

$$
R_{0}^{m} f=\sum_{n=0}^{\infty} P_{m} f_{m+n}, \quad m=0,1, \ldots
$$

and so $C R_{0}^{m} f=f_{m}$ for $m=0,1, \ldots$ To prove (8.8) we write

$$
S=S(0)+P_{1} \odot R_{0} S .
$$

Writing $S=\sum_{n=0}^{\infty} P_{n} S_{n}$ we conclude by applying (8.7) to $R_{0} S u$ for $u \in \mathbb{H}^{s}$.

Remark 8.4. A deep difference with respect to the classical case is that the kernel functions of $\mathbf{H}_{2}(\mathcal{E})$ are not eigenvectors for $R_{0}$.

Note that $(8.8)$ is not equal to $D+C \odot\left(I-P_{1} A\right)^{-\odot} \odot B$. But, for $x_{1}=x_{2}=x_{3}=0$ we have

$$
S\left(x_{0}\right)=D+3 x_{0} C\left(I-3 x_{0} A\right)^{-1} B .
$$

Remark 8.5. Following linear system theory (see $[31,60,67,78]$ ) we will call the representation (8.8) a realization of $S$. The associated matrix $\widehat{V}$ will be called the realization matrix or the Rosenbrock matrix. The case where $\widehat{V}^{*}$ is a matrix can be seen as the definition of rational functions. Unfortunately, the $C K$-product of two such functions will not be rational in this sense. The next section deals with an important example of rational functions. 


\subsection{Blaschke Functions}

Equation (8.8) allows us to give a family of Schur multipliers, which we call Blaschke functions, namely those corresponding to the operator-matrix (8.6) to be a unitary matrix. The definition then extends the classical case, also in the matrix-valued and possibly indefinite case; see [17] for the latter. In general there will not be $\odot$-multiplicative decompositions of such a Blaschke "product" into elementary factors, hence the term function rather than product.

Proposition 8.6. Let $B$ be $\mathbb{H}^{r \times r}$-valued Blaschke function, with corresponding realization matrix $\widehat{V}^{*} \in \mathbb{H}^{(N+r) \times(N+r)}$. The corresponding multiplication operator $M_{B}$ is an isometry from $\left(\mathbf{H}_{2}(\mathcal{E})\right)^{r}$ into itself and the corresponding space $\mathcal{H}(B)$ is finite dimensional.

Proof. We first remark that the assumed unitarity is equivalent to the equations

$$
\begin{aligned}
& \mathrm{A}^{*} \mathrm{~A}+\mathrm{C}^{*} \mathrm{C}=I_{N}, \\
& \mathrm{~B}^{*} \mathrm{~B}+\mathrm{D}^{*} \mathrm{D}=I_{r}, \\
& \mathrm{D}^{*} \mathrm{C}+\mathrm{B}^{*} \mathrm{~A}=0 .
\end{aligned}
$$

Let now $n_{0}, m_{0} \in \mathbb{N}_{0}$, and $u, v \in \mathbb{H}^{r}$. We have, with $b_{n}$ given by (8.7),

$$
b_{n}= \begin{cases}\mathrm{D}, & \text { if } n=0, \\ \mathrm{CA}^{\mathrm{n}-1} \mathrm{~B}, & \text { if } n>0,\end{cases}
$$

and so

$$
\begin{aligned}
\left\langle M_{B} P_{n_{0}} u, M_{B} P_{m_{0}} v\right\rangle_{2} & =\left\langle P_{n_{0}} \odot B u, P_{m_{0}} \odot B v\right\rangle_{2} \\
& =\sum_{n, m=0}^{\infty}\left\langle P_{n_{0}+n} b_{n} u, P_{m_{0}+m} b_{m} v\right\rangle_{2} \\
& =\sum_{\substack{n, m=0 \\
n_{0}+n=m_{0}+m}}^{\infty} v^{*} b_{m}^{*} b_{n} u
\end{aligned}
$$

We now compute

$$
\sum_{\substack{n, m=0 \\ n_{0}+n=m_{0}+m}}^{\infty} b_{m}^{*} b_{n}
$$


taking into account that the matrix $\widehat{V}$ is unitary. When $n_{0}=m_{0}$ we have

$$
\begin{aligned}
\sum_{n=0}^{\infty} b_{n}^{*} b_{n} & =\mathrm{D}^{*} \mathrm{D}+\sum_{n=0}^{\infty} \mathrm{B}^{*} \mathrm{~A}^{* n} \mathrm{C}^{*} \mathrm{CA}^{n} \mathrm{~B} \\
& =\mathrm{D}^{*} \mathrm{D}+\sum_{n=0}^{\infty} \mathrm{B}^{*} \mathrm{~A}^{* n}\left(I_{N}-\mathrm{A}^{*} \mathrm{~A}\right) \mathrm{A}^{n} \mathrm{~B} \\
& =\mathrm{D}^{*} \mathrm{D}+\sum_{n=0}^{\infty} \mathrm{B}^{*}\left(\mathrm{~A}^{* n} \mathrm{~A}^{n}-\mathrm{A}^{*(n+1)} \mathrm{A}^{n+1}\right) \mathrm{B} \\
& =\mathrm{D}^{*} \mathrm{D}+\mathrm{B}^{*} \mathrm{~B} \\
& =I_{r},
\end{aligned}
$$

where we first used (8.9) and then (8.10).

Assume now $n_{0}<m_{0}$ (the case $n_{0}>m_{0}$ is obtained by symmetry). We can write:

$$
\begin{aligned}
\sum_{\substack{n, m=0 \\
n_{0}+n=m_{0}+m}}^{\infty} b_{m}^{*} b_{n} & =\sum_{m=0}^{\infty} b_{m}^{*} b_{m_{0}-n_{0}+m} \\
& =\mathrm{D}^{*} \mathrm{CA} \mathrm{A}^{m_{0}-n_{0}} \mathrm{~B}+\sum_{m=1}^{\infty} \mathrm{B}^{*} \mathrm{~A}^{* m} \mathrm{C}^{*} \mathrm{CA}^{m} \mathrm{~A}^{m_{0}-n_{0}} \mathrm{~B} \\
& =\mathrm{D}^{*} \mathrm{CA}^{m_{0}-n_{0}} \mathrm{~B}+\sum_{m=1}^{\infty} \mathrm{B}^{*} \mathrm{~A}^{* m}\left(I_{N}-\mathrm{A}^{*} \mathrm{~A}\right) \mathrm{A}^{m} \mathrm{~A}^{m_{0}-n_{0}} \mathrm{~B} \\
& =\mathrm{D}^{*} \mathrm{CA} \mathrm{A}^{m_{0}-n_{0}} \mathrm{~B}+\mathrm{B}^{*} \mathrm{~A} \mathrm{~A}^{m_{0}-n_{0}} \mathrm{~B} \\
& =\left(\mathrm{D}^{*} \mathrm{C}+\mathrm{B}^{*} \mathrm{~A}\right) \mathrm{A}^{m_{0}-n_{0}} \mathrm{~B} \\
& =0
\end{aligned}
$$

in view of $(8.11)$.

We thus have an isometry on the linear span of $P_{0}, P_{1}, \ldots$ and on the whole of $\left(\mathbf{H}_{2}(\mathcal{E})\right)^{r}$ by continuity.

To show the finite dimensionality of the space we restrict $x=x_{0}, y=$ $y_{0} \in(-1 / 3,1 / 3)$. We then have

$$
K_{S}\left(3 x_{0}, 3 y_{0}\right)=\frac{I_{r}-S\left(x_{0}\right) S\left(y_{0}\right)^{*}}{1-9 x_{0} y_{0}}=\mathrm{C}\left(I_{N}-3 x_{0} \mathrm{~A}\right)^{-1}\left(I_{N}-3 y_{0} \mathrm{~A}\right)^{-1} \mathrm{C}^{*} .
$$

It follows that the linear span of the functions $x_{0} \mapsto K_{S}\left(3 x_{0}, 3 y_{0}\right) h\left(h \in \mathbb{H}^{r}\right.$ and $y_{0} \in(-1 / 3,1.3)$ is finite dimensional. By the uniqueness of the axially hyperholomorphic extension the linear span of the functions $x \mapsto K_{S}\left(x, 3 y_{0}\right) h$ is finite dimensional We claim that they span $\mathcal{H}(S)$. Indeed, a function $f \in \mathcal{H}(S)$ orthogonal to these functions would satisfy $f\left(3 y_{0}\right)=0$ for $y_{0} \in$ $(-1 / 3,1 / 3)$. Since $f$ is of axial type we have that $f \equiv 0$.

The above computations show equivalently that:

Corollary 8.7. Let $\widehat{V}^{*}$ given by (8.6). Then the corresponding Toeplitz operator defined by the sequence (8.12) is unitary from $\ell_{2}\left(\mathbb{N}_{0}, \mathbb{H}^{r}\right)$ into itself. 
More generally, one can take $\widehat{V}^{*}$ to be $\mathbb{C}^{(N+s) \times(N+s)}$-valued and coisometric. Then (8.9)-(8.11) still hold and the same proof as above leads to:

Theorem 8.8. Let $S(x)=\mathrm{D}+\sum_{n=1}^{\infty} P_{n}(x) \mathrm{CA}^{n-1} \mathrm{~B}$, where the realization matrix (8.6) is coisometric. Then the corresponding operator $M_{S}$ is an isometry from $\left(\mathbf{H}_{2}(\mathcal{E})\right)^{s}$ into $\left(\mathbf{H}_{2}(\mathcal{E})\right)^{r}$.

Remark 8.9. Take $S_{1}, \ldots, S_{N}$ to be $N$ Schur multipliers (say, $\mathbb{H}$-valued) with associated finite dimensional $\mathcal{H}\left(S_{j}\right)$ spaces, $j=1, \ldots, N$, and let $t_{1}, \ldots, t_{N}$ to be real numbers such that $\sum_{n=1}^{N} t_{n}^{2}=1$. The function

$$
S(x)=\left(t_{1} S_{1}(x) \cdots t_{N} S_{N}(x)\right)
$$

is a Schur multiplier from $\left(\mathbf{H}_{2}(\mathcal{E})\right)^{N}$ into $\mathbf{H}_{2}(\mathcal{E})$, and the associated reproducing kernel space is finite dimensional, but will not be isometrically included in $\mathbf{H}_{2}(\mathcal{E})$ when $N>1$; see [16, p. 71] for the complex setting. The argument is the same here.

Remark 8.10. In fact the results in the present section still hold when $\widehat{V}^{*}$ is isometric, but not necessarily a matrix. Then the corresponding multiplier is called inner. The study of these multipliers will be considered elsewhere. Similarly, one could assume unitarity with respect to an indefinite metric. This aspect of the theory will also be treated in a separate publication.

\subsection{Rational Functions}

We now define rational functions in the present setting. We first recall that any $\mathbb{C}^{n \times m}$-valued rational function, say $M(z)$, with no pole at the origin can be written in the form

$$
M(z)=H+z G(I-z T)^{-1} F,
$$

where $H, G, T, F$ are matrices of appropriate sizes. Expression (8.13) is called a realization (centered at the origin). See Remark 8.5 above for references. We also recall the formulas for the product and inverse of rational functions. Note that, since we consider possibly non-square functions, the sum will be a special case of the product since

$$
\left(M_{1}(z) I_{n}\right)\left(\begin{array}{c}
I_{m} \\
M_{2}(z)
\end{array}\right)=M_{1}(z)+M_{2}(z)
$$

where $M_{1}$ and $M_{2}$ are $\mathbb{C}^{n \times m}$-valued.

Assuming in (8.13) that $n=m$ and $H$ invertible, one has the formula

$$
M(z)^{-1}=H^{-1}-z H^{-1} G\left(I-z T^{\times}\right)^{-1} F H^{-1},
$$

where $T^{\times}=T-G H^{-1} F$, and with $M_{j}(z)=H_{j}+z G_{j}\left(I-z T_{j}\right)^{-1} F_{j}, j=1,2$, two rational functions of compatible sizes,

$$
M_{1}(z) M_{2}(z)=H+z G(I-z T)^{-1} F,
$$

with $H=H_{1} H_{2}$ and

$$
T=\left(\begin{array}{cc}
T_{1} & G_{1} T_{2} \\
0 & T_{2}
\end{array}\right), \quad G=\left(\begin{array}{c}
G_{1} \\
H_{1} G_{2}
\end{array}\right), \quad F=\left(\begin{array}{ll}
F_{1} H_{2} & F_{2}
\end{array}\right) .
$$


Definition 8.11. The $\mathbb{H}^{r \times s}$-valued function $R(x)$ hyperholomorphic of axial type is called rational if its restriction to the real axis is a rational function of the real variable, with quaternionic coefficients.

Theorem 8.12. The $\mathbb{H}^{r \times s}$-valued function $R(x)$ hyperholomorphic of axial type, and defined at the origin, is rational if and only if $x_{0} \mapsto R\left(3 x_{0}\right)$ can be written as

$$
R\left(3 x_{0}\right)=\mathrm{D}+3 x_{0} \mathrm{C}\left(I-3 x_{0} \mathrm{~A}\right)^{-1} \mathrm{~B}
$$

where A, B, C, B are quaternionic matrices of appropriate sizes.

Equivalently:

Theorem 8.13. The $\mathbb{H}^{r \times s}$-valued function $R(x)$ hyperholomorphic of axial type, and defined at the origin, is rational if and only if it can be written as

$$
R(x)=\mathrm{D}+\sum_{n=0}^{\infty} P_{n}(x) \mathrm{CA}^{n-1} \mathrm{~B}
$$

where A, B, C, B are quaternionic matrices of appropriate sizes.

We will not give the proofs of the above results, which follow easily from the previous analysis in the paper. One still has the formulas (8.14) and (8.15) for a real variable $x_{0}$, but not for the $C K$-product. So the product of rational hyperholomorphic functions of axial type is not compatible with the $C K$ product. To emphasize this point, we now make the connection with rational functions of the Fueter variables, as studied in [10,20,23] (see also [71], and see [18] for the split quaternionic case). There, rational functions are characterized by the formula (we do not specify the sizes of the various quaternionic matrices)

$$
\begin{aligned}
R(x) & =\mathrm{D}+\mathrm{C} \odot\left(I-\left(\zeta_{1} \mathrm{~A}_{1}+\zeta_{2} \mathrm{~A}_{2}+\zeta_{3} \mathrm{~A}_{3}\right)^{-\odot} \odot\left(\zeta_{1} \mathrm{~B}_{1}+\zeta_{2} \mathrm{~B}_{2}+\zeta_{3} \mathrm{~B}_{3}\right)\right. \\
& =\mathrm{D}+\mathrm{C} \odot(I-\zeta \mathrm{A})^{-\odot} \odot(\zeta \mathrm{B}),
\end{aligned}
$$

with

$$
A=\left(\begin{array}{l}
A_{1} \\
A_{2} \\
A_{3}
\end{array}\right) \quad \text { and } B=\left(\begin{array}{l}
B_{1} \\
B_{2} \\
B_{3}
\end{array}\right),
$$

and the variable here is

$$
\zeta=\left(\zeta_{1} \zeta_{2} \zeta_{3}\right)
$$

We look at the special case where

$$
\mathrm{A}=\left(\begin{array}{c}
\mathbf{e}_{1} \mathscr{A} \\
\mathbf{e}_{2} \mathscr{A} \\
\mathbf{e}_{3} \mathscr{A}
\end{array}\right) \quad \text { and } \quad \mathrm{B}=\left(\begin{array}{c}
\mathbf{e}_{1} \mathscr{B} \\
\mathbf{e}_{2} \mathscr{B} \\
\mathbf{e}_{3} \mathscr{B}
\end{array}\right)
$$


where $\mathscr{A}$ and $\mathscr{B}$ are matrices of appropriate sizes and with quaternionic coefficients. Since $P_{1}(x)=\zeta_{1}(x) \mathbf{e}_{1}+\zeta_{2}(x) \mathbf{e}_{2}+\zeta_{3}(x) \mathbf{e}_{3}$ we can then rewrite (8.18) as

$$
R(x)=\mathrm{D}+\mathrm{C} \odot\left(I-P_{1}(x) \mathscr{A}\right)^{-\odot} \odot P_{1}(x) \mathscr{B},
$$

which will not be in general a series in the $P_{n}$, but is a series in the $\zeta^{\alpha}$. We can define such elements as the rational functions associated with the polynomials $P_{n}$. Then, things make sense in terms of realizations, with the usual formulas, but we do not get power series in $P_{n}$, even when $\mathcal{A}$ is nilpotent.

\subsection{A Structure Theorem}

In the classical, and scalar-valued, setting, Beurling's theorem asserts that a closed subspace $\mathcal{N}$ of the Hardy space $\mathbf{H}_{2}(\mathbb{D})$ is invariant under multiplication by the complex variable if and only if it is of the form $\mathcal{N}=j \mathbf{H}_{2}(\mathbb{D})$, where $j$ is an inner function. The space $\mathcal{M}=\mathbf{H}_{2}(\mathbb{D}) \ominus \mathcal{N}$ is then isometrically included in $\mathbf{H}_{2}(\mathbb{D})$, backward-shift invariant, and has reproducing kernel equal to $\frac{1-j(z) \overline{j(w)}}{1-z \bar{w}}$. One motivation for the theory of de Branges-Rovnyak is to characterize reproducing kernel Hilbert spaces of functions which are contractively included in $\mathbf{H}_{2}(\mathbb{D})$, and $R_{0}$-invariant. Rather than the latter, one assumes that the inequality

$$
\left\|R_{0} f\right\|_{\mathcal{M}}^{2} \leq\|f\|_{\mathcal{M}}^{2}-|f(0)|^{2}
$$

holds. This inequality implies contractive inclusion in the Hardy space,

$$
f \in \mathcal{M} \Longrightarrow f \in \mathbf{H}_{2}(\mathbb{D}) \text { and }\|f\|_{\mathbf{H}_{2}(\mathbb{D})} \leq\|f\|_{\mathcal{M}}
$$

and that in particular the space is a reproducing kernel Hilbert space since

$$
|f(w)| \leq \frac{1}{\sqrt{1-|w|^{2}}}\|f\|_{\mathbf{H}_{2}(\mathbb{D})} \leq \frac{1}{\sqrt{1-|w|^{2}}}\|f\|_{\mathcal{M}}, \quad w \in \mathbb{D} .
$$

Theorem 8.14. Let $\mathcal{M}$ be a Hilbert space of functions analytic in the open unit disk and such that (8.19) holds in $\mathcal{M}$. Then, $\mathcal{M}$ is contractively included inside $\mathbf{H}_{2}(\mathbb{D})$ and there is a (possibly $\ell_{2}$-valued) analytic function s such that the reproducing kernel of $\mathcal{N}$ is $\frac{1-s(z) s(w)^{*}}{1-z \bar{w}}$.

We refer to the notes in [11, p. 206] for some history on Theorem 8.14, but mention the papers [72]. Guyker characterized the spaces for which the inclusion is isometric; see [11, p. 187], [64,65]. For an illustration of the contractive inclusion, see Remark 8.9 above.

A general version of Theorem 8.14, in the operator-valued and Pontryagin space case, has been proved in [11, Theorem 3.1.2, p. 85]. We will present in a subsequent paper the general version of the result, in the Pontryagin and operator-valued function case. The purpose of this section is to illustrate the power of the methods used here on a simple case. Note that (8.21) is a weakening of (4.10).

Theorem 8.15. Let $\mathcal{H}$ be a Hilbert space of $\mathbb{H}^{r}$-valued functions axially hyperholomorphic in $\mathcal{E}$, and $R_{0}$-invariant and satisfying

$$
\left\|R_{0} f\right\|_{\mathcal{H}}^{2} \leq\|f\|_{\mathcal{H}}^{2}-f(0)^{*} f(0), \quad f \in \mathcal{H} .
$$


Then there exist a right quaternionic Hilbert space $\mathcal{C}$ and a $\mathbf{L}\left(\mathcal{C}, \mathbb{H}^{r}\right)$-valued function $S$ hyperholomorphic of axial type such that $\mathcal{H}=\mathcal{H}(S)$.

Proof. It follows from (8.21) that $\mathcal{H}$ is contractively included in $\left(\mathbf{H}_{2}(\mathcal{E})\right)^{r}$, and that the operator $R_{0}$ is bounded (another argument, still valid in the quaternionic Pontryagin space setting would be to prove that $R_{0}$ is closed, thanks to the reproducing kernel property, and use the closed graph theorem; see [8, Theorem 5.1.16, p. 74] for the latter). The point evaluation at the origin, which we will denote by $C$, is also bounded since the space is contractively included in the Hardy space and its norm is larger than the Hardy space norm. We can thus rewrite (8.21) as

$$
R_{0}^{*} R_{0}+C^{*} C \leq I
$$

Since the adjoint of a contraction between Hilbert space is still a contraction we have

$$
I_{\mathcal{H} \oplus \mathbb{H}^{r}}-\left(\begin{array}{c}
R_{0} \\
C
\end{array}\right)\left(\begin{array}{c}
R_{0} \\
C
\end{array}\right)^{*} \geq 0,
$$

and we can factorize the quaternionic positive operator via a right quaternionic Hilbert space $\mathcal{C}$ as

$$
I_{\mathcal{H} \oplus \mathbb{H}^{r}}-\left(\begin{array}{c}
R_{0} \\
C
\end{array}\right)\left(\begin{array}{c}
R_{0} \\
C
\end{array}\right)^{*}=\left(\begin{array}{c}
B \\
D
\end{array}\right)\left(\begin{array}{c}
B \\
D
\end{array}\right)^{*},
$$

with

$$
\left(\begin{array}{l}
B \\
D
\end{array}\right) \in \mathbf{L}\left(\mathcal{C}, \mathcal{H} \oplus \mathbb{H}^{r}\right)
$$

The operator matrix

$$
\left(\begin{array}{cc}
R_{0} & B \\
C & D
\end{array}\right): \mathcal{H} \oplus \mathcal{C} \longrightarrow \mathcal{H} \oplus \mathbb{H}^{r}
$$

is co-isometric, and the $\mathbf{L}\left(\mathcal{C}, \mathbb{H}^{r}\right)$-valued function $S$ defined by

$$
S(x)=D+\sum_{n=1}^{\infty} P^{\odot n}(x) C R_{0}^{n} B
$$

is a Schur multiplier. To conclude we show that $\mathcal{H}=\mathcal{H}(S)$. From the definition of $S$ we have for $x_{0}, y_{0} \in(-1 / 3,1 / 3)$

$$
K_{S}\left(3 x_{0}, 3 y_{0}\right)=C\left(I-3 x_{0} R_{0}\right)^{-1}\left(I-3 y_{0} R_{0}\right)^{-*} C^{*} .
$$

We have

$$
K_{S}\left(3 x_{0}, 3 y_{0}\right)-3 x_{0} K_{S}\left(3 x_{0}, 3 y_{0}\right) 3 y_{0}=I_{r}-S\left(3 x_{0}\right) S\left(3 y_{0}\right)^{*}
$$

and the result follows from axially hyperholomorphic extension on the left with respect to $x$ and on the right with respect to $y$. 


\section{Spaces $\mathcal{L}(\Phi)$}

We now consider the counterpart of $\mathcal{L}(\Phi)$ spaces, see (1.2), in the present setting, and first briefly review the classical case. Functions analytic in the open unit disk and with a positive real part there will be called here Herglotz functions (they are called Carathéodory functions in Akhiezer's book [5, p. 116]). An Herglotz function, say $\Phi$, is characterized by an integral representation of the form

$$
\Phi(z)=i m+\int_{[0,2 \pi]} \frac{e^{i t}+z}{e^{i t}-z} d \sigma(t)
$$

where $m \in \mathbb{R}, \sigma$ is an increasing function, and (9.1) is a Stieltjes integral. They play an important role in the trigonometric moment problem, operators models for isometric and unitary operators in Hilbert spaces and in the theory of dissipative discrete systems, and have been extended to various more general frameworks; see e.g. [1,27,29,30,68,69,73].

In a way similar to Theorem 2.21, consider a function $\Phi$ defined in a real neighborhood $(-\epsilon, \epsilon)$ of the origin and such that the kernel

$$
\frac{\Phi(a)+\overline{\Phi(b)}}{2(1-a b)}
$$

is positive definite there. Then it is the restriction to $(-\epsilon, \epsilon)$ of a uniquely defined Herglotz function, and the corresponding kernel

$$
L_{\Phi}(z, w)=\frac{\Phi(z)+\overline{\Phi(w)}}{2(1-z \bar{w})}
$$

is positive definite in the open unit disk. The factor 2 in the kernels is to get nicer realization formulas (such as (9.2)) and follows basically from the Cayley transform $z \mapsto \frac{1-z}{1+z}$, which maps Herglotz functions into Schur functions.

The corresponding reproducing kernel Hilbert space and its applications to operator models was first characterized and studied by de Branges; see $[41,45]$. It is important to note that a Herglotz function need not be bounded, and hence need not be a multiplier of the Hardy space.

Using the reproducing kernel space $\mathcal{L}(\Phi)$ associated with $L_{\Phi}$ (or directly from (9.1)), one can characterize Herglotz functions in terms of a realization of the form

$$
\Phi(z)=i a+C(I+z V)(I-z V)^{-1} C^{*},
$$

where $V$ is coisometric in some Hilbert space, and $C^{*}$ is a continuous map from the coefficient space (the complex numbers when the functions are scalar) into that Hilbert space. Note that (9.2) can be rewritten as

$$
\Phi(z)=i a+C C^{*}+2 \sum_{n=1}^{\infty} z^{n} C V^{n} C^{*}, \quad z \in \mathbb{D} .
$$

In this section we study the counterpart of the kernel $L_{\Phi}$ in our setting, and give the counterpart of the expansion (9.3), and study connections with Toeplitz operators. In the following definition (and also in Sect. 12 below) we 
use the term multiplier although the operator of $C K$-multiplication by the given function need not be bounded in the Hardy space.

Definition 9.1. A $\mathbb{H}^{r \times r}$-valued axially hyperholomorphic function $\Phi$ is called a Herglotz multiplier if the kernel

$$
L_{\Phi}(x, y)=\frac{1}{2} \sum_{n=0}^{\infty}\left(P_{n} \odot \Phi\right)(x) P_{n}(y)^{*}+P_{n}(x)\left(\left(P_{n} \odot \Phi\right)(y)\right)^{*}
$$

is positive definite in $\mathcal{E}$.

When the operator of $C K$-multiplication by $\Phi$ is bounded in the Hardy space $\left(\mathbf{H}_{2}(\mathcal{E})\right)^{r}$, we can replace $(9.4)$ by the condition (see Remark 4.18)

$$
\Gamma \stackrel{\text { def. }}{=} \frac{M_{\Phi}+M_{\Phi}^{*}}{2} \geq 0
$$

Proposition 9.2. We note the following property of $\Gamma$ :

$$
M_{P_{1}} \Gamma M_{P_{1}}^{*}=\frac{1}{2}\left\{M_{\Phi}\left(I-C^{*} C\right)+\left(I-C^{*} C\right) M_{\Phi}^{*}\right\} .
$$

Proof. Using the fundamental equality (4.10), we can write

$$
\begin{aligned}
M_{P_{1}} \Gamma M_{P_{1}}^{*} & =M_{P_{1}}\left(\frac{M_{\Phi}+M_{\Phi}^{*}}{2}\right) M_{P_{1}}^{*} \\
& =\frac{1}{2} M_{\Phi} M_{P_{1}} M_{P_{1}}^{*}+\frac{1}{2} M_{P_{1}} M_{P_{1}}^{*} M_{\Phi}^{*} \\
& =\frac{1}{2} M_{\Phi}\left(I-C^{*} C\right)+\frac{1}{2}\left(I-C^{*} C\right) M_{\Phi}^{*} .
\end{aligned}
$$

We make now some remarks on the above kernel. It seems difficult to find a direct counterpart of (9.2) [in view of (2.24)], let alone of (9.1). As for the case of Schur multiplier, we look for a characterization of the coefficients of $\Phi$ in its expansion along the $P_{n}$. When $x=x_{0}$ and $y=y_{0}$ belong to $(-1 / 3,1 / 3)$ the kernel $L_{\Phi}$ becomes

$$
L_{\Phi}\left(3 x_{0}, 3 y_{0}\right)=\frac{\Phi\left(3 x_{0}\right)+\Phi\left(3 y_{0}\right)^{*}}{1-9 x_{0} y_{0}} .
$$

As for the case of Schur multipliers, this restriction is enough to get back the kernel $L_{\Phi}$ in view of the axial symmetry of the functions.

Definition 9.3. We denote by $\mathcal{L}(\Phi)$ the reproducing kernel Hilbert space of axially hyperholomorphic functions with reproducing kernel (9.4).

The Hilbert space $\mathcal{L}(\Phi)$ is the completion of the linear span of functions of the form $L_{\Phi}(x, y) p, p \in \mathbb{H}^{r}$, thus it consists of hyperholomorphic functions of axial type. 
Theorem 9.4. Let $\Phi(x)=\sum_{n=0}^{\infty} P_{n}(x) \Phi_{n}$ be an axially hyperholomophic function in $\mathcal{E}$. Then $\Phi$ is a Herglotz multiplier if and only if the coefficients $\Phi_{n}$ can be written as

$$
\Phi_{n}=\left\{\begin{array}{l}
C C^{*}, \quad n=0 \\
2 C V^{* n} C^{*}, \quad n=1,2, \ldots
\end{array}\right.
$$

where $V$ is an isometry in a Hilbert space, say $\mathfrak{H}$, and $C$ is a continuous map from $\mathfrak{H}$ into $\mathbb{H}^{r}$.

Proof. We first prove the sufficiency. We have for $a \in(-1,1)$

$$
\Phi(a)=C C^{*}+2 \sum_{n=1}^{\infty} a^{n} C V^{* n} C^{*}=C\left(I+a V^{*}\right)\left(I-a V^{*}\right)^{-1} C^{*} .
$$

Thus, for $a, b \in(-1,1)$,

$$
\frac{\Phi(a)+\Phi(b)^{*}}{2(1-a b)}=C\left(I-a V^{*}\right)^{-1}\left(I-b V^{*}\right)^{-*} C^{*}
$$

and thus the corresponding function (9.7) is positive definite in $(-1 / 3,1 / 3)$, and we get the result by hyperholomorphic extension on the left with respect to $x$ and on the right with respect to $y$ since the functions are assumed axially hyperholomorphic.

We now turn to the proof of the direct statement, and divide the proof in a number of steps. We follow [25, Proof of Theorem 5.2, p. 708]. We write $P_{1}\left(3 y_{0}\right)$ rather than $3 y_{0}$ to emphasize the axially symmetric hypercomplex extension to be used. At the end of section alternative steps 1 and 2 are given when $M_{\Phi}$ is bounded.

STEP 1: The linear relation (see Definition 3.1) of $\mathcal{L}(\Phi) \times \mathcal{L}(\Phi)$ defined by the span of the functions

$$
\left(L_{\Phi}\left(\cdot, 3 y_{0}\right) \overline{P_{1}\left(3 y_{0}\right)} q,\left(L_{\Phi}\left(\cdot, 3 y_{0}\right)-L_{\Phi}(\cdot, 0)\right) q\right), \quad y_{0} \in(-1 / 3,1 / 3), \quad q \in \mathbb{H}^{r},
$$

is densely defined and isometric.

Let $F$ be orthogonal to the domain of the relation, then $P_{1}\left(3 y_{0}\right) F\left(3 y_{0}\right) \equiv$ 0 and so $F$ is identically equal to 0 by axially hypercomplex extension.

Next, we need to show that, for $x_{0}, y_{0}, \in(-1 / 3,1 / 3)$ and $p, q \in \mathbb{H}^{r}$, we have:

$$
\begin{aligned}
\left\langle L_{\Phi}\left(\cdot, 3 y_{0}\right) \overline{P_{1}\left(3 y_{0}\right)} q, L_{\Phi}\left(\cdot, 3 x_{0}\right) \overline{P_{1}\left(3 x_{0}\right)} p\right\rangle_{\mathcal{L}(\Phi)} & \\
=\left\langle\left(L_{\Phi}\left(\cdot, 3 y_{0}\right)-L_{\Phi}(\cdot, 0)\right) q,\left(L_{\Phi}\left(\cdot, 3 x_{0}\right)\right.\right. & \left.\left.-L_{\Phi}(\cdot, 0)\right) p\right\rangle_{\mathcal{L}(\Phi)} .
\end{aligned}
$$

This amounts to check that

$$
P_{1}\left(3 x_{0}\right) L_{\Phi}\left(3 x_{0}, 3 y_{0}\right) \overline{P_{1}\left(3 y_{0}\right)}=L_{\Phi}\left(3 x_{0}, 3 y_{0}\right)-L_{\Phi}\left(3 x_{0}, 0\right)-L_{\Phi}\left(0,3 y_{0}\right)+L_{\Phi}(0,0)
$$

but this is a direct consequence of the definition of $L_{\Phi}$ since

$$
L_{\Phi}\left(3 x_{0}, 0\right)+L_{\Phi}\left(0,3 y_{0}\right)-L_{\Phi}(0,0)=\frac{\Phi\left(3 x_{0}\right)+\Phi\left(3 y_{0}\right)^{*}}{2}
$$


and

$$
L_{\Phi}\left(3 x_{0}, 3 y_{0}\right)-P_{1}\left(3 x_{0}\right) L_{\Phi}\left(3 x_{0}, 3 y_{0}\right) \overline{P_{1}\left(3 y_{0}\right)}=\frac{\Phi\left(3 x_{0}\right)+\Phi\left(3 y_{0}\right)^{*}}{2} .
$$

There is thus an everywhere defined isometric operator such that, for $q \in \mathbb{H}^{r}$

$$
V\left(L_{\Phi}\left(\cdot, 3 y_{0}\right) \overline{P_{1}\left(3 y_{0}\right)} q\right)=\left(L_{\Phi}\left(\cdot, 3 y_{0}\right)-L_{\Phi}(\cdot, 0)\right) q, \quad y_{0} \in(-1 / 3,1 / 3) .
$$

STEP 2: We have

$$
V^{*}=R_{0}
$$

On the one hand

$$
\begin{aligned}
\left.\left\langle V^{*} F,\left(L_{\Phi}\left(\cdot, 3 y_{0}\right) \overline{P_{1}\left(3 y_{0}\right)}\right) q\right)\right\rangle_{\mathcal{L}(\Phi)} & =\left\langle F,\left(L\left(\cdot, 3 y_{0}\right)-L(\cdot, 0)\right) q\right\rangle_{\mathcal{L}(\Phi)} \\
& =q^{*}\left(F\left(3 y_{0}\right)-F(0)\right)
\end{aligned}
$$

and on the other hand

$$
\begin{aligned}
\left\langle V^{*} F,\left(L_{\Phi}\left(\cdot, 3 y_{0}\right) \overline{P_{1}\left(3 y_{0}\right)} q\right)\right\rangle_{\mathcal{L}(\Phi)} & \left.=\left\langle F,\left(L_{\Phi}\left(\cdot, 3 y_{0}\right) \overline{P_{1}\left(3 y_{0}\right)}\right) q\right)\right\rangle_{\mathcal{L}(\Phi)} \\
& =q^{*} P_{1}\left(3 y_{0}\right)\left(V^{*} F\right)\left(3 y_{0}\right) .
\end{aligned}
$$

Hence,

$$
P_{1}\left(3 y_{0}\right)\left(V^{*} F\right)\left(3 y_{0}\right)=F\left(3 y_{0}\right)-F(0),
$$

and hence the result by axially hypercomplex extension.

STEP 3: Let $C$ denote the evaluation at the origin in $\mathcal{L}(\Phi)$. It holds that

$$
\left(C^{*} q\right)(x)=L_{\Phi}(x, 0) q=\left(\Phi(x)+\Phi(0)^{*}\right) q, \quad q \in \mathbb{H}^{r} .
$$

We have, with $p, q \in \mathbb{H}^{r}$,

$$
\left\langle C^{*} q, L_{\Phi}(\cdot, x) p\right\rangle_{\mathcal{L}(\Phi)}=\langle q, L(0, x) p\rangle_{\mathbb{H}^{r}}=\frac{1}{2}\left(\left(\Phi(0)+\Phi(x)^{*}\right) p\right)^{*} q
$$

and hence the result.

STEP 4: We prove (9.8).

With $q \in \mathbb{H}^{r}$ we write

$$
\begin{aligned}
\Phi(x) q & =\left(\Phi(x)+\Phi(0)^{*}\right) q-\Phi(0)^{*} q \\
& =2\left(C^{*} q\right)(x)-\Phi(0)^{*} q \\
& =2 \sum_{n=0}^{\infty} C V^{* n} C^{*} q-\Phi(0)^{*} q \\
& =C C^{*}+2 \sum_{n=1}^{\infty} C V^{* n} C^{*} q+C C^{*}-\Phi(0)^{*} q \\
& =C C^{*}+2 \sum_{n=1}^{\infty} C V^{* n} C^{*} q+\frac{\Phi(0)-\Phi(0)^{*}}{2} q
\end{aligned}
$$

since $C C^{*}=\frac{\Phi(0)+\Phi(0)^{*}}{2}$.

Corollary 9.5. Let $\Phi$ be a Herglotz multiplier. The space $\mathcal{L}(\Phi)$ is $R_{0}$ invariant.

As for Schur multiplier one has: 
Theorem 9.6. A space $\mathcal{L}(\Phi)$ is finite dimensional if and only if the operator $V$ can be chosen to act in a finite dimensional space (and hence is unitary).

In terms of Toeplitz matrices we have:

Theorem 9.7. $\Phi$ is a $\mathbb{H}^{r \times r}$-valued bounded Herglotz multiplier if and only if the infinite block matrix $\left(\Phi_{n-m}\right)_{n, m=0}^{\infty}$ (with $\Phi_{-m}=\Phi_{m}^{*}$ ) defines a bounded positive operator.

Proof. Let $\Phi$ be such that the kernel $L_{\Phi}$ is positive definite in $\mathcal{E}$ (at this stage we do not assume yet that the associated operator $M_{\Phi}$ is bounded). Then, setting $x_{1}=x_{2}=x_{3}=0$ and applying the map $\chi$ we see that the kernel

$$
\frac{\chi(\Phi)\left(3 x_{0}\right)+\left(\chi(\Phi)\left(3 y_{0}\right)\right)^{*}}{1-9 x_{0} y_{0}}
$$

is positive definite in $(-1 / 3,1 / 3)$. Setting $a=3 x_{0}$ and $b=3 y_{0}$, and with $\varphi(a)=\chi(\Phi)\left(3 x_{0}\right)$ we see that the kernel

$$
\frac{\varphi(a)+\varphi(b)^{*}}{1-a b}
$$

is positive definite on $(-1,1)$. Thus it extends to a positive definite function to the open unit disk, and the extended $\varphi$ has a positive real part there. The block Toeplitz matrices

$$
\left(\chi\left(\Phi_{n-m}\right)\right)_{n, m=0}^{N},
$$

with $\Phi_{-m}=\Phi_{m}^{*}$, are thus non-negative. If $M_{\Phi}$ is bounded, the infinite Toeplitz matrix $\left(\chi\left(\Phi_{n-m}\right)\right)_{n, m=0}^{\infty}$ defines a bounded positive operator, and so does $\left(\Phi_{n-m}\right)_{n, m=0}^{\infty}$ by Proposition 2.2.

Conversely, if $\left(\Phi_{n-m}\right)_{n, m=0}^{\infty}$ defines a bounded positive operator, the function

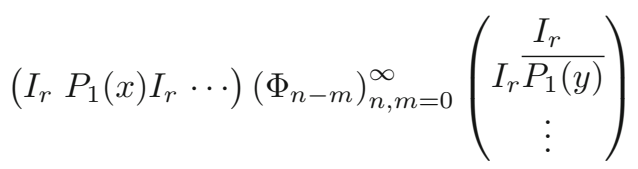

is positive definite. It can be rewritten as the kernel $L_{\Phi}$. Indeed

$$
\left(\begin{array}{lll}
I_{r} & P_{1}(x) I_{r} \cdots
\end{array}\right)\left(\Phi_{n-m}\right)_{n, m=0}^{\infty}\left(\begin{array}{c}
I_{r} \frac{I_{r}}{P_{1}(y)} \\
\vdots
\end{array}\right)=\sum_{n, m=0}^{\infty} P_{n}(x) \Phi_{n-m} \overline{P_{m}(y)}
$$

while

$$
\begin{aligned}
L_{\Phi}(x, y) & =\sum_{n=0}^{\infty}\left(\sum_{m=0}^{\infty} P_{n+m}(x) \Phi_{m}\right) \overline{P_{m}(y)}+P_{n}(x)\left(\sum_{m=0}^{\infty} \Phi_{m}^{*} \overline{P_{m+n}(y)}\right) \\
& =\sum_{n=0}^{\infty} \sum_{m=0}^{\infty} P_{n}(x) \Phi_{n-m} \overline{P_{m}(y)}
\end{aligned}
$$

with $\Phi_{-m}=\Phi_{m}^{*}$ for $m=0,1, \ldots$ 
We conclude with a computation of the linear relation associated to $\Phi$ when $M_{\Phi}$ is a bounded operator. The computations are longer, but avoid axially symmetric extensions. The relation (9.9) becomes:

$$
\left(\sum_{j} \Gamma\left(M_{P_{1}}^{*} k_{\mathcal{E}}\left(\cdot, a_{j}\right) q_{j}\right), \sum_{j} L_{\Phi}\left(\cdot, a_{j}\right) q_{j}-L_{\Phi}(\cdot, 0)\left(\sum_{j} q_{j}\right)\right),
$$

where $u_{1}, v_{1} \ldots \in \mathcal{E}$ and $p_{1}, q_{1}, \ldots \in \mathbb{H}^{r}$.

NEW STEP 1: The linear relation spanned by the elements (9.10) is isometric and densely defined, and hence extends to the graph of an everywhere defined isometry.

To prove this claim, we let $f=\sum_{j} k_{\mathcal{E}}\left(\cdot, u_{j}\right) q_{j}$ and $g=\sum_{\ell} k_{\mathcal{E}}\left(\cdot, v_{\ell}\right) p_{\ell}$. Using (9.6) we can write:

$$
\begin{aligned}
\left\langle\Gamma\left(M_{P_{1}}^{*} f\right), \Gamma\left(M_{P_{1}}^{*} g\right)\right\rangle_{\mathcal{L}(\Phi)}= & \left\langle M_{P_{1}}^{*} f, \Gamma\left(M_{P_{1}}^{*} g\right)\right\rangle_{2} \\
= & \left\langle f, M_{P_{1}} \Gamma\left(M_{P_{1}}^{*} g\right)\right\rangle_{2} \\
= & \frac{1}{2}\left\langle f,\left(M_{\Phi}\left(I-C^{*} C\right)+\left(I-C^{*} C\right) M_{\Phi}^{*}\right) g\right\rangle_{2} \\
= & \langle f, \Gamma g\rangle-\frac{1}{2}\left(C M_{\Phi}^{*} g\right)^{*} C f-\frac{1}{2}(C g)^{*} C M_{\Phi}^{*} f \\
= & \sum_{j, \ell} p_{\ell}^{*} L_{\Phi}\left(v_{\ell}, u_{j}\right) q_{j}-\frac{1}{2}\left(\sum_{\ell} \Phi\left(v_{\ell}\right)^{*} p_{\ell}\right)^{*}\left(\sum_{j} q_{j}\right) \\
& -\frac{1}{2}\left(\sum_{\ell} p_{\ell}\right)^{*}\left(\sum_{j} \Phi\left(u_{j}\right)^{*} q_{j}\right) \\
= & \sum_{j, \ell} p_{\ell}^{*} L_{\Phi}\left(v_{\ell}, u_{j}\right) q_{j}-\left(\sum_{\ell} L_{\Phi}\left(0, v_{\ell}\right)^{*} p_{\ell}\right)^{*}\left(\sum_{j} q_{j}\right) \\
& -\left(\sum_{\ell} p_{\ell}\right)^{*}\left(\sum_{j} L_{\Phi}\left(0, u_{j}\right)^{*} q_{j}\right) \\
& +\left(\sum_{\ell} p_{\ell}\right)^{*} L_{\Phi}(0,0)\left(\sum_{j} L_{\Phi}\left(0, u_{j}\right)^{*} q_{j}\right) \\
& =\left\langle\sum_{j} L_{\Phi}\left(\cdot, u_{j}\right) q_{j}-L_{\Phi}(\cdot, 0)\left(\sum_{j} q_{j}\right),\right. \\
& \left.\sum_{\ell} L_{\Phi}\left(\cdot, v_{\ell}\right) p_{\ell}-L_{\Phi}(\cdot, 0)\left(\sum_{\ell} p_{\ell}\right)\right\rangle_{\mathcal{L}(\Phi)} .
\end{aligned}
$$

There is thus an everywhere defined isometric operator such that

$$
V\left(\Gamma M_{P_{1}}^{*} k_{\mathcal{E}}(\cdot, u) q\right)=L_{\Phi}(\cdot, u) q-L_{\Phi}(\cdot, 0) q, \quad u \in \mathcal{E} .
$$

NEW STEP 2: We have

$$
V^{*}=R_{0}
$$


Indeed, let $V^{*} F=\sqrt{\Gamma} f$. On the one hand,

$$
\begin{aligned}
\left\langle V^{*} F, \sqrt{\Gamma}\left(M_{P_{1}}^{*} k_{\mathcal{E}}(\cdot, u)\right) q\right\rangle_{\mathcal{L}(\Phi)} & =\left\langle\sqrt{\Gamma} f, M_{P_{1}}^{*} k_{\mathcal{E}}(\cdot, u) q\right\rangle_{2} \\
& =\left\langle M_{P_{1}} V^{*} F, k_{\mathcal{E}}(\cdot, u) q\right\rangle_{2} \\
& =q^{*}\left(P_{1} \odot V^{*} F\right)(u) .
\end{aligned}
$$

On the other hand,

$$
\begin{aligned}
\left\langle V^{*} F, \sqrt{\Gamma}\left(M_{P_{1}}^{*} k_{\mathcal{E}}(\cdot, u)\right) q\right\rangle_{\mathcal{L}(\Phi)} & =\left\langle F, V\left(\sqrt{\Gamma}\left(M_{P_{1}}^{*} k_{\mathcal{E}}(\cdot, u)\right) q\right)\right\rangle_{\mathcal{L}(\Phi)} \\
& =\left\langle F, L_{\Phi}(\cdot, u) q-L_{\Phi}(\cdot, 0) q\right\rangle_{\mathcal{L}(\Phi)} \\
& =q^{*}(F(u)-F(0)) .
\end{aligned}
$$

Hence, using the formulas (7.4)-(7.5) for the operator range inner product, we have

$$
\left(P_{1} \odot V^{*} F\right)(u)=F(u)-F(0) .
$$

\section{The Half-Space Case}

We first recall that the classical Hardy space of the open right half-plane $\mathbb{C}_{r}$ is the reproducing kernel Hilbert space with reproducing kernel equal to $\frac{1}{2 \pi(z+\bar{w})}$ (the factor $2 \pi$ appears because of Cauchy's formula), and can be characterized as the space of power series of the form

$$
b(z)=\sum_{n=0}^{\infty} \frac{(1-z)^{n}}{(1+z)^{n+1}} b_{n}
$$

where the complex numbers $b_{n}$ satisfy $\sum_{n=0}^{\infty}\left|b_{n}\right|^{2}<\infty$; see for instance [66]. We will denote this space by $\mathbf{H}_{2}\left(\mathbb{C}_{r}\right)$. The purpose of this section is to define and begin a study of the counterpart of the space $\mathbf{H}_{2}\left(\mathbb{C}_{r}\right)$ in the present setting. A more detailed analysis will be presented in a sequel to the present work. We give three equivalent characterizations of the new space, respectively in terms of a reproducing kernel, restriction to the positive real axis and series expansion analogous to (10.1). We first define what will be the counterpart of $\mathbb{C}_{r}$. To that purpose, consider the function $w(x)$ defined in (1.3); it has for (unique) $C K$-extension

$$
W_{1}(x)=\left(1-P_{1}(x)\right) \odot\left(1+P_{1}(x)\right)^{-\odot} .
$$

This function is intrinsic hyperholomorphic of axial type by Remark 2.14, in fact in a neighborhood of the origin we can write it as

$$
\begin{gathered}
W_{1}(x)=\left(1-P_{1}(x)\right) \odot \sum_{n=0}^{\infty}(-1)^{n} P_{1}(x)^{\odot n}=\sum_{n=0}^{\infty}(-1)^{n} P_{1}(x)^{\odot n} \\
+\sum_{n=0}^{\infty}(-1)^{n+1} P_{1}(x)^{\odot n+1} \\
=1+2 \sum_{n=1}^{\infty}(-1)^{n} P_{1}(x)^{\odot n} .
\end{gathered}
$$


We define $W_{n}(x)=W_{1}^{\odot n}(x)$ and we set

$$
K_{W}(x, y)=\sum_{n=0}^{\infty} W_{n}(x) \overline{W_{n}(y)} .
$$

Note that $W_{n}(0)=1$ and also that $W_{n}(x)$ is hyperholomorphic of axial type, being a finite $C K$-product of intrinsic series in $P_{1}(x)$. On the other hand for $x_{1}=x_{2}=x_{3}=0$ and $x_{0}>0$,

$$
\left|W_{n}\left(x_{0}\right)\right|=\left|\frac{1-3 x_{0}}{1+3 x_{0}}\right|<1 .
$$

Using the arguments in Lemma 2.3 we can prove the following:

Lemma 10.1. The series

$$
\sum_{n=0}^{\infty}\left|W_{n}(x)\right|^{2}
$$

converges in a neighborhood of $x=1 / 3$.

Proof. The function $W_{1}(x)$ is hyperholomorphic by its definition. Since we have $W_{1}(1 / 3)=0$ we consider the variable $\tilde{x}=x-\frac{1}{3}$. The composed function $W_{1}(1 / 3+\tilde{x})=\tilde{W}_{1}(\tilde{x})$ is still hyperholomorphic, $\tilde{W}_{1}(0)=0$ and we can consider its expansion at the origin

$$
\tilde{W}_{1}(\tilde{x})=\sum_{\substack{\alpha \in \mathbb{N}_{0}^{3} \\ \alpha \neq(0,0,0)}} \zeta(\tilde{x})^{\alpha} f_{\alpha} .
$$

By Lemma 2.3 we have that for any $\rho>0$ there exists $\epsilon>0$ such that for $\tilde{x}_{0}^{2}+\tilde{x}_{j}^{2}<\epsilon, j=1,2,3$, one has

$$
\left|\left(\tilde{W}_{1}(\tilde{x})\right)^{\odot n}\right|<\rho^{n}, \quad n=1,2, \ldots .
$$

Since $\tilde{W}_{n}(\tilde{x})=W_{n}(1 / 3+x)=\left(W_{1}(1 / 3+x)\right)^{\odot n}=\left(\tilde{W}_{1}(\tilde{x})^{\odot n}\right.$ we deduce that $\left|\tilde{W}_{n}(\tilde{x})\right|<\rho^{n}$ for $\tilde{x}_{0}^{2}+\tilde{x}_{j}^{2}<\epsilon, j=1,2,3$. Thus, for any $0<\rho<1$ there exists $\epsilon>0$ such that

$$
\sum_{n=0}^{\infty}\left|W_{n}(x)\right|^{2}<\sum_{n=0}^{\infty} \rho^{2 n}
$$

for $\left(x_{0}-1 / 3\right)^{2}+x_{j}^{2}<\epsilon, j=1,2,3$ and the statement follows.

Definition 10.2. We denote by $\mathcal{P}$ the subset of $\mathbb{H}$ for which the series

$$
\sum_{n=0}^{\infty}\left|W_{n}(x)\right|^{2}
$$

converges.

The set $\mathcal{P}$ is nonempty as the previous lemma shows, moreover we have:

Proposition 10.3. The $\mathcal{P}$ contains all the points of the positive real axis $\mathbb{R}^{+}$ and for any $\tilde{x}_{0} \in \mathbb{R}^{+}$there exists a neighborhood of $\tilde{x}_{0}$ in which the series converges. 
Proof. Let us consider a point $\tilde{x}_{0} \in \mathbb{R}^{+}$, then $W_{1}\left(\tilde{x}_{0}\right)=w_{1} \in \mathbb{R}$ with $\left|w_{1}\right|<1$. Let $\tilde{x}=x-\tilde{x}_{0}$ and set $W_{1}\left(\tilde{x}+\tilde{x}_{0}\right)=\breve{W}_{1}(\tilde{x})$. Since $\breve{W}_{1}(0)=w_{1}$ we write $\breve{W}_{1}(\tilde{x})=w_{1}+\tilde{W}_{1}(x)$ with $\tilde{W}_{1}(0)=0$. Let us set $\breve{W}_{n}(\tilde{x})=\left(\breve{W}_{1}(\tilde{x})\right)^{\odot n}$ then

$$
\breve{W}_{n}(\tilde{x})=\left(\breve{W}_{1}(\tilde{x})\right)^{\odot n}=\left(w_{1}+\tilde{W}_{1}(\tilde{x})\right)^{\odot n}=\sum_{k=0}^{n}\left(\begin{array}{l}
n \\
k
\end{array}\right) \tilde{W}_{1}(\tilde{x})^{\odot k} w_{1}^{n-k},
$$

so that

$$
\left|\breve{W}_{n}(\tilde{x})\right| \leq \sum_{k=0}^{n}\left(\begin{array}{l}
n \\
k
\end{array}\right)\left|\tilde{W}_{1}(\tilde{x})\right|^{k}\left|w_{1}\right|^{n-k} .
$$

The proof of Lemma 10.1 shows that for any $0<\rho<1$ there exists $\epsilon>0$ such that $\left|\tilde{W}_{1}(\tilde{x})\right|^{k}<\rho^{k}$ for $\left(x-\tilde{x}_{0}\right)^{2}+x_{j}^{2}<\epsilon, j=1,2,3$. Setting $\eta=1-\left|w_{1}\right|>0$, we take $\rho=\eta / 2$ so that we have

$$
\left|\breve{W}_{n}(\tilde{x})\right| \leq\left(\left|w_{1}\right|+\rho\right)^{n}=(1-\eta / 2)^{n}
$$

and $\left|W_{n}(x)\right|^{2}<(1-\eta / 2)^{n}$ in a neighborhood of $\tilde{x}_{0}$. The assertion follows.

As in Sect. $2, K_{W}(x, y)$ solves the Eq. (8.1), with $W_{1}$ replacing $P_{1}$, namely

$$
K_{W}(x, y)-W_{1}(x) \odot K_{W}(x, y) \odot_{r} \overline{W_{1}(y)}=1 .
$$

We set (note that we do not put a factor $2 \pi$ ):

$$
K_{\mathcal{P}}(x, y)=\left(1+P_{1}(x)\right)^{-\odot} \odot K_{W}(x, y) \odot_{r}\left(1+\overline{P_{1}(y)}\right)^{-\odot_{r}} .
$$

Theorem 10.4. The kernel $K_{\mathcal{P}}(x, y)$ is positive definite in $\mathcal{P}$ and is the unique solution of the Lyapunov equation

$$
2\left(P_{1}(x) \odot K_{\mathcal{P}}(x, y)+K_{\mathcal{P}}(x, y) \odot_{r} \overline{P_{1}(y)}\right)=1 .
$$

Proof. The first claim follows from the formula

$$
\left.\left.K_{\mathcal{P}}(x, y)=\sum_{n=0}^{\infty}\left(1+P_{1}(x)\right)^{-\odot} \odot W_{n}(x)\right)\left(1+P_{1}(y)\right)^{-\odot_{r}} \odot_{r} W_{n}(y)\right)^{*}
$$

where we have used Proposition 2.20 relating the left and right $C K$-products.

We have from (10.6)

$$
K_{W}(x, y)=\left(1+P_{1}(x)\right) \odot K_{\mathcal{P}}(x, y) \odot_{r}\left(1+\overline{P_{1}(y)}\right) .
$$

Moreover, we have

$$
\left(1+P_{1}(x)\right) \odot\left(1-P_{1}(x)\right) \odot\left(1+P_{1}(x)\right)^{-\odot}=\left(1-P_{1}(x)\right) .
$$

Using these equations we rewrite (10.5) as

$$
\begin{aligned}
& \left(1+P_{1}(x)\right) \odot K_{\mathcal{P}}(x, y) \odot_{r}\left(1+\overline{P_{1}(y)}\right) \\
& \quad-\left(1-P_{1}(x)\right) \odot K_{\mathcal{P}}(x, y) \odot_{r}\left(1+\overline{P_{1}(y)}\right)=1
\end{aligned}
$$

from which we get (10.7). 
We denote by $\mathbf{H}_{2}(\mathcal{P})$ the reproducing kernel Hilbert space with reproducing kernel equal to $K_{\mathcal{P}}(x, y)$. We also note that (10.7) and (10.11) are equivalent, but (10.11) is better adapted to use the lurking isometry method or the linear relation method, when one considers multipliers (see Sect. 11). The counterpart of the expansion (10.1) is presented in the following theorem.

Theorem 10.5. The reproducing kernel Hilbert space associated with the kernel $K_{\mathcal{P}}(x, y)$ consists of the power series

$$
f(x)=\sum_{n=0}^{\infty}\left(1+P_{1}(x)\right)^{-\odot} \odot W_{n}(x) f_{n},
$$

where the coefficients $f_{n}$ are in $\mathbb{H}$ and satisfy $\sum_{n=0}^{\infty}\left|f_{n}\right|^{2}<\infty$. The latter is then the square of the norm of $f$.

Proof. This follows from (10.8).

We now turn to the characterization of $\mathbf{H}_{2}(\mathcal{P})$ in terms of restrictions to the real positive axis.

Theorem 10.6. $f \in \mathbf{H}_{2}(\mathcal{P})$ if and only if $x_{0} \mapsto \chi\left(f\left(3 x_{0}\right)\right)$ is the restriction to $x_{0}>0$ of an element in $\left(\mathbf{H}_{2}\left(\mathbb{C}_{r}\right)\right)^{2 \times 2}$. The map which to $f \in \mathbf{H}_{2}(\mathcal{P})$ associates the map $x_{0} \mapsto \sqrt{\pi} \chi\left(f\left(x_{0}\right)\right)$ is then unitary.

Proof. Setting $x_{1}=x_{2}=x_{3}=0$ we get

$$
f\left(x_{0}\right)=\sum_{n=0}^{\infty} \frac{\left(1-3 x_{0}\right)^{n}}{\left(1+3 x_{0}\right)^{n+1}} f_{n} .
$$

Applying the map $\chi$ and comparing with (10.1) we get the statement. The function is uniquely determined since $(0, \infty)$ is a zero set. The converse follows from the fact that $x_{0} \mapsto \frac{\left(1-3 x_{0}\right)^{n}}{\left(1+3 x_{0}\right)^{n+1}}$ has as unique axially hyperholomorphic extension the function $\left(1+P_{1}(x)\right)^{-\odot} \odot W_{n}(x)$ (which is evidently of axial type), being the $C K$-product of series in $P_{1}(x)$.

\section{Schur Multipliers in the Half-Plane Setting}

In the classical case of the complex numbers, a $\mathbb{C}^{r \times r}$-valued function is contractive in $\mathbb{C}_{r}$ if and only if the kernel

$$
\frac{I_{r}-s(z) s(w)^{*}}{z+\bar{w}}
$$

is positive definite in $\mathbb{C}_{r}$. More generally, if a function $s$ is defined in a zero set, say $\mathcal{Z}$, of the open right half-plane and the kernel (11.1) is positive definite on $\mathcal{Z}$, then $s$ is the restriction to $\mathcal{Z}$ of a uniquely defined function analytic and contractive in $\mathbb{C}_{r}$. This can be seen from the disk case (see Theorem 2.21) using a Cayley transform. In the present section we study the counterpart of the Schur multipliers for the space $\mathbf{H}_{2}(\mathcal{P})$, and characterize them in three equivalent ways:

(1) In terms of a positive definite kernel. 
(2) In terms of an appropriately defined multiplication operator.

(3) In terms of a realization.

Definition 11.1. A $\mathbb{H}^{r \times r}$-valued $S$ function is called a Schur multiplier if there is a kernel $K_{S}(x, y)$ positive definite in $\mathcal{P}$, left-hyperholomophic in $x$ and right-hyperholomorphic in $y$, and such that

$$
\begin{aligned}
& 2\left(P_{1}(x) \odot K_{S}(x, y)\right. \\
& \left.\quad+K_{S}(x, y) \odot_{r} \overline{P_{1}(y)}\right)=I_{r}-S(x) S(y)^{*}, \quad x, y \in \mathcal{P} .
\end{aligned}
$$

We will get a description of all such multipliers in terms of a realization, but already mention a very easy example, namely $S(x)=W_{1}(x)$. Then, with

$$
K_{S}(x, y)=\left(1+P_{1}(x)\right)^{-\odot}\left(1+\overline{P_{1}(y)}\right)^{-\odot_{r}}
$$

we have

$$
2\left(P_{1}(x) \odot K_{S}(x, y)+K_{S}(x, y) \odot_{r} \overline{P_{1}(y)}\right)=1-W_{1}(x) \overline{W_{1}(y)}, \quad x, y \in \mathcal{P} .
$$

In view of the lurking isometry method, it is better to write (11.2) as

$$
\begin{aligned}
(1+ & \left.P_{1}(x)\right) \odot K_{S}(x, y) \odot_{r}\left(1+\overline{P_{1}(y)}\right) \\
& \quad-\left(1-P_{1}(x)\right) \odot K_{S}(x, y) \odot_{r}\left(1-\overline{P_{1}(y)}\right) \\
= & I_{r}-S(x) S(y)^{*}
\end{aligned}
$$

or as

$$
\begin{aligned}
(1 & \left.+P_{1}(x)\right) \odot K_{S}(x, y) \odot_{r}\left(1+\overline{P_{1}(y)}\right)+S(x) S(y)^{*} \\
& =\left(1-P_{1}(x)\right) \odot K_{S}(x, y) \odot_{r}\left(1-\overline{P_{1}(y)}\right)+I_{r} .
\end{aligned}
$$

Theorem 11.2. The $\mathbb{H}^{r \times s}$-valued function $S$ is a Schur multiplier if and only if there exist a Hilbert space $\mathcal{H}$ and a co-isometry

$$
\left(\begin{array}{ll}
A & C \\
B & D
\end{array}\right)
$$

such that

$$
S\left(3 x_{0}\right)=D+\frac{1-3 x_{0}}{1+3 x_{0}} C\left(I-\frac{1-3 x_{0}}{1+3 x_{0}} A\right)^{-1} B, \quad x_{0} \in(-1 / 3,1 / 3),
$$

with unique hyperholomorphic extension (of axial type) to $\mathcal{P}$ given by

$$
S(x)=D+\sum_{n=0}^{\infty} W_{n}(x) C A^{n} B .
$$

Proof. Write

$$
K_{S}(x, y)=X(x) X(y)^{*}
$$

where $X$ is operator-valued and hyperholomorphic of axial type (for instance, via the associated reproducing kernel Hilbert space; one takes $X(x)$ to be the 
point evaluation at $x$ ). We get the isometric relation (the lurking isometry) spanned by the pairs

$$
\left(\left(\begin{array}{c}
X\left(3 y_{0}\right)^{*}\left(1-\overline{P_{1}\left(3 y_{0}\right)}\right) h \\
h
\end{array}\right),\left(\begin{array}{c}
X\left(3 y_{0}\right)^{*}\left(1+\overline{P_{1}\left(3 y_{0}\right)}\right) h \\
S\left(3 y_{0}\right)^{*} h
\end{array}\right)\right)
$$

with $y_{0} \in(-1 / 3,1 / 3)$ and $h \in \mathbb{H}^{s}$. Write the isometry as

$$
\left(\begin{array}{ll}
A^{*} & B^{*} \\
C^{*} & D^{*}
\end{array}\right)
$$

We get

$$
\begin{aligned}
\frac{1-3 y_{0}}{1+3 y_{0}} A^{*} X\left(y_{0}\right)^{*} h+\frac{1}{1+3 y_{0}} C^{*} h & =X\left(y_{0}\right)^{*} h \\
\left(1-3 y_{0}\right) B^{*} h+\sqrt{2} D^{*} h & =S\left(y_{0}\right)^{*} h
\end{aligned}
$$

Hence,

$$
S\left(3 y_{0}\right)^{*}=D^{*}+\frac{1-3 y_{0}}{1+3 y_{0}} B^{*}\left(I-\frac{1-3 y_{0}}{1+3 y_{0}} A^{*}\right)^{-1} C^{*}
$$

and hence the result, since $W_{n}(x)$ is the unique hyperholomorphic extension of axial type.

In the complex setting case, a function, say $s$, analytic and contractive in $\mathbb{C}_{r}$ does not need belong to $\mathbf{H}^{2}\left(\mathbb{C}_{r}\right)$, but $z \mapsto s(z) /(1+z)$ does belong to $\mathbf{H}^{2}\left(\mathbb{C}_{r}\right)$. Here, at least in the present analysis, we need a supplementary condition to get the counterpart of this result. We have (the notion of spectral radius is defined for a quaternionic operator $A$ as in the classical case by the formula $\left.\rho(A)=\lim \sup _{n \rightarrow \infty}\left\|A^{n}\right\|^{1 / n}\right)$ :

Corollary 11.3. In the notation of Theorem 11.2, assume $\rho(A)<1$. Then, the entries of the function $\left(1+P_{1}(x)\right)^{-\odot} \odot S(x)$ belong to $\mathbf{H}_{2}(\mathcal{P})$.

Proof. This follows from the fact that

$$
\left\|C A^{n} B\right\| \leq\|C\| \cdot\|B\| \cdot\left\|A^{n}\right\|,
$$

and the series $\sum_{n=1}^{\infty}\left\|A^{n}\right\|$ converges since $\rho(A)<1$.

Theorem 11.4. $S$ is a Schur multiplier if and only if the operator defined by

$$
M_{S} f=\sum_{n=0}^{\infty}\left(1+P_{1}(x)\right)^{-\odot}\left(W_{n} \odot S\right)(x) f_{n}
$$

is a contraction from $\mathbf{H}_{2}(\mathcal{P})$ into itself, and $K_{S}$ is given by

$$
\begin{aligned}
K_{S}(x, y)= & \left(1+P_{1}(x)\right)^{-\odot} \odot \\
& \odot\left(\sum_{n=0}^{\infty} W_{n}(x) \overline{W_{n}(y)}-\sum_{n=0}^{\infty}\left(W_{n} \odot S\right)(x) \overline{\left(W_{n} \odot S\right)(y)}\right) \\
& \odot_{r}\left(1+\overline{P_{1}(y)}\right)^{-\odot_{r} .}
\end{aligned}
$$


Proof. We consider the scalar case to ease the notation and first remark that, if $S$ is a Schur multiplier we have

$$
K_{S}\left(3 x_{0}, 3 y_{0}\right)=\frac{1-S\left(3 x_{0}\right) \overline{S\left(3 y_{0}\right)}}{3\left(x_{0}+y_{0}\right)}
$$

with axially hyperholomorphic extension (11.9), and the positivity of (11.9) expresses that the operator $M_{S}$ is a contraction. The converse is proved by defining a contractive relation from the positivity of the kernel, and show that the relation extends to the graph of $M_{S}^{*}$.

As in the $\mathcal{E}$ setting, the case where the isometry in the above realizations is unitary in a finite dimensional space corresponds to finite dimensional $\mathcal{H}(S)$ spaces isometrically included in the Hardy space $\left(\mathbf{H}_{2}(\mathcal{P})\right)^{r}$. When the space has dimension 1 the function $S$ is the counterpart of a Blaschke factor of the half-plane.

Theorem 11.5. The space $\mathcal{H}(S)$ is finite dimensional if and only if the space $\mathfrak{H}$ can be chosen finite dimensional. When $r=s$, the space $\mathcal{H}(S)$ is isometrically included inside $\left(\mathbf{H}_{2}(\mathcal{P})\right)^{r}$.

Proof. We set

$$
\left(\begin{array}{ll}
A & B \\
C & D
\end{array}\right)
$$

the matrix of the underlying unitary map. We have:

$$
\begin{aligned}
M_{S} & \left(\left(1+P_{1}(x)\right)^{-\odot} \odot W_{m}(x) h\right) \\
& =\left(1+P_{1}(x)\right)^{-\odot} \odot W_{m}(x) D h+\sum_{n=0}^{\infty}\left(1+P_{1}(x)\right)^{-\odot} \odot W_{m+n}(x) \mathrm{CA}^{n} \mathrm{Bh} .
\end{aligned}
$$

The same computations as in the proof of Proposition 8.6 show that

$$
\begin{aligned}
\left\langle M_{S}\right. & \left.\left.\left.\left(1+P_{1}(x)\right)^{-\odot} \odot W_{n_{1}} h(x)\right), M_{S}\left(1+P_{1}(x)\right)^{-\odot} \odot W_{n_{2}}(x) k\right)\right\rangle \\
= & \sum_{\substack{n, m=0 \\
n_{1}+n=n_{2}+m}}^{\infty} k^{*} \mathrm{~B}^{*} \mathrm{~A}^{* n} \mathrm{AC}^{*} \mathrm{C}^{m} \mathrm{Bh} \\
= & \delta_{n, m} k^{*} h, \quad h, k \in \mathbb{H}^{s},
\end{aligned}
$$

and this allows to end the proof.

\section{Carathéodory Multipliers in the Half-Plane Setting}

A function $\Phi$ analytic and with a positive real part in the open right-half plane is called a Carathéodory function, and is characterized by the positivity of the kernel

$$
\frac{\Phi(z)+\overline{\Phi(w)}}{z+\bar{w}}
$$

in $\mathbb{C}_{r}$. As for Herglotz functions, a Carathéodory function need not be a multiplier of the Hardy space $\mathbf{H}_{2}\left(\mathbb{C}_{r}\right)$. We now introduce the counterpart 
of this class of functions in the present setting. As in Sect. 9 we use the term multiplier (rather than, for instance pseudo-multiplier) although the $C K$-multiplication by the function $\Phi$ is not assumed bounded.

Definition 12.1. An $\mathbb{H}^{r \times r}$-valued function $\Phi$ is called a Carathéodory pseudomultiplier if there is a kernel $K_{\Phi}(x, y)$ positive definite in $\mathcal{P}$, left-hyperholomophic in $x$ and right-hyperholomorphic in $y$, and such that

$$
2\left(P_{1}(x) \odot K_{\Phi}(x, y)+K_{\Phi}(x, y) \odot_{r} \overline{P_{1}(y)}\right)=\Phi(x)+\Phi(y)^{*} .
$$

A first example is given by $\Phi(x)=a P_{1}(x)$ with $a>0$ and $K_{\Phi}(x, y)=$ $a / 2$. It follows from the definition that a sum of Carathéodory multipliers is a Carathéodory multiplier, and so is $\Phi^{-\odot}$ and $a \Phi$ with $a>0$. Therefore, any sum of the form

$$
\Phi(x)=a_{0} P_{1}(x)+\sum_{n=1}^{N} b_{n}\left(a_{n}+P_{1}(x)\right)^{-\odot}
$$

is a Carathéodory multiplier for every choice of $a_{0} \geq 0, a_{1}, \ldots, a_{N}>0$ and $b_{1}, \ldots, b_{N} \geq 0$.

It is more convenient to rewrite (12.1) as

$$
\begin{aligned}
(1 & \left.+P_{1}(x)\right) \odot K_{\Phi}(x, y) \odot_{r}\left(1+\overline{P_{1}(y)}\right)-\left(1-P_{1}(x)\right) \odot K_{\Phi}(x, y) \odot\left(1-\overline{P_{1}(y)}\right. \\
& =\left(I_{r}+\Phi(x)\right)\left(I_{r}+\Phi(y)^{*}\right)-\left(I_{r}-\Phi(x)\right)\left(I_{r}-\Phi(y)^{*}\right) .
\end{aligned}
$$

Theorem 12.2. $\Phi$ is a Carathéodory multiplier if and only if it can be written as

$$
\Phi\left(3 y_{0}\right)=\left(I_{r}-S\left(3 y_{0}\right)\right)\left(I_{r}+S\left(3 y_{0}\right)\right)^{-1}
$$

with $S$ as in (11.6).

Proof. Write

$$
K_{\Phi}(x, y)=X(x) X(y)^{*}
$$

where $X(x)$ is the point evaluation in the reproducing kernel Hilbert space with reproducing kernel $K_{\Phi}$. We get the isometric relation (the lurking isometry) defined by the right linear span of the pairs

$$
\left(\left(\begin{array}{c}
X\left(3 y_{0}\right)^{*}\left(1-\overline{P_{1}\left(3 y_{0}\right)}\right) h \\
\left(I_{r}+\Phi\left(3 y_{0}\right)^{*}\right) h
\end{array}\right),\left(\begin{array}{c}
X\left(3 y_{0}\right)^{*}\left(1+\overline{P_{1}\left(3 y_{0}\right)}\right) h \\
\left(I_{r}-\Phi\left(3 y_{0}\right)^{*}\right) h
\end{array}\right)\right),
$$

with $y_{0} \in(-1 / 3,1 / 3)$ and $h \in \mathbb{H}^{r}$. Furthermore, with the same notation as (11.8),

$$
\begin{aligned}
\frac{1-3 y_{0}}{1+3 y_{0}} A^{*} X\left(3 y_{0}\right)^{*} h+\frac{1}{1+3 y_{0}} C^{*}\left(I_{r}+\Phi\left(3 y_{0}\right)^{*}\right) h & =X^{*}\left(3 y_{0}\right) h, \\
\left(1-3 y_{0}\right) B^{*} X^{*}\left(3 y_{0}\right) h+D^{*}\left(\left(I_{r}+\Phi\left(3 y_{0}\right)^{*}\right) h\right. & =\left(I-\Phi\left(3 y_{0}\right)^{*}\right) h,
\end{aligned}
$$

and hence

$$
\begin{aligned}
& \left(I_{r}-\Phi\left(3 y_{0}\right)^{*}\right) h-D^{*}\left(I_{r}+\Phi\left(3 y_{0}\right)\right)^{*} h=\frac{1-3 y_{0}}{1+3 y_{0}} B^{*}\left(I-\frac{1-3 y_{0}}{1+3 y_{0}} A^{*}\right)^{-1} \\
& \quad \times C^{*}\left(I_{r}+\Phi\left(3 y_{0}\right)\right)^{*} h
\end{aligned}
$$


so that, with $S\left(3 y_{0}\right)$ as in the previous theorem,

$$
I_{r}-\Phi\left(3 y_{0}\right)^{*}=S\left(3 y_{0}\right)^{*}\left(I_{r}+\Phi\left(3 y_{0}\right)^{*}\right)
$$

and hence the result.

Here too, the space $\mathcal{L}(\Phi)$ will be finite dimensional if and only if the space $\mathfrak{H}$ can be chosen finite dimensional.

\section{A Table}

We conclude this paper with a table comparing the slice hyperholomorphic case, the case of Fueter variables and the present setting.

\begin{tabular}{|c|c|c|c|}
\hline & Slice setting & Appell setting & General $C K$-setting \\
\hline variable & $p \in \mathbb{H}$ & $\begin{array}{l}P_{1}(x)=C K\left(x_{1} \mathbf{e}_{1}+x_{2} \mathbf{e}_{2}+x_{3} \mathbf{e}_{3}\right) \\
\quad=\zeta_{1}(x) \mathbf{e}_{1}+\zeta_{2}(x) \mathbf{e}_{2}+\zeta_{3}(x) \mathbf{e}_{3}\end{array}$ & $\begin{array}{l}\zeta(x)= \\
=\left(\zeta_{1}(x) \zeta_{2}(x) \zeta_{3}(x)\right)\end{array}$ \\
\hline "unit disk" & $x_{0}^{2}+x_{1}^{2}+x_{2}^{2}+x_{3}^{2}<1$ & $9 x_{0}^{2}+x_{1}^{2}+x_{2}^{2}+x_{3}^{2}<1$ & $3 x_{0}^{2}+x_{1}^{2}+x_{2}^{2}+x_{3}^{2}<1$ \\
\hline product & $\star$-product & $C K$-product & $C K$-product \\
\hline properties & stays inside the space & $\begin{array}{l}\text { outside the space } \\
\text { Not a power series in } P_{n}\end{array}$ & stays inside the space \\
\hline inverse & $\begin{array}{l}(I-p A)^{-\star}= \\
=\sum_{n=0}^{\infty} p^{n} A^{n}\end{array}$ & $\begin{array}{c}\qquad\left(I-P_{1} A\right)^{-\odot} \\
\text { outside the space } \\
\text { Not a power series in } P_{n}\end{array}$ & $\begin{array}{c}(I-\zeta A)^{-\odot}= \\
=\sum_{\alpha \in \mathbb{N}_{0}^{3}} \frac{|\alpha !|}{\alpha !} \zeta^{\alpha} A^{\alpha}\end{array}$ \\
\hline $\begin{array}{c}\text { Hardy or } \\
\text { Drury-Arveson } \\
\text { reproducing kernel }\end{array}$ & $k(p, q)=\sum_{n=0}^{\infty} p^{n} \bar{q}^{n}$ & $\sum_{n=0}^{\infty} P_{n}(x) \overline{P_{n}(y)}$ & $\begin{array}{c}k_{y}(x)= \\
=\sum_{\alpha \in \mathbb{N}_{0}^{3}} \frac{|\alpha !|}{\alpha !} \zeta^{\alpha}(x) \overline{\zeta^{\alpha}(y)}\end{array}$ \\
\hline structural identity & $I-M_{p} M_{p}^{*}=C^{*} C$ & $\begin{array}{c}I-M_{P_{1}} M_{P_{1}}^{*}=C^{*} C \\
\text { (but does not hold in } Q_{n} \text { setting) }\end{array}$ & $I-M_{\zeta} \mathcal{M}_{\zeta}^{*}=C^{*} C$ \\
\hline $\begin{array}{l}\text { multiplication } \\
\text { operator }\end{array}$ & $\begin{array}{l}\text { Cauchy product on } \\
\text { coefficients }\end{array}$ & $\begin{array}{l}M_{S}\left(\sum_{n=0}^{\infty} P_{n} u_{n}\right)= \\
=\sum_{n=0}^{\infty}\left(P_{n} \odot S\right) u_{n}\end{array}$ & $\odot$-multiplication \\
\hline $\begin{array}{c}\text { adjoint of } \\
\text { multiplication } \\
\text { operator }\end{array}$ & $\begin{aligned} & M_{S}^{*} k(p, q)= \\
= & \sum_{n=0}^{\infty} p^{n} \overline{S(q)} \bar{q}^{n}\end{aligned}$ & $\begin{array}{l}M_{S}^{*}\left(\sum_{n=0}^{\infty} P_{n} \overline{P_{n}(a)} u\right)= \\
=\sum_{n=0}^{\infty} P_{n} \overline{\left(P_{n} \odot S\right)(a)} u\end{array}$ & $\begin{array}{c}M_{S}^{*} k_{y}(x)= \\
=\sum_{\alpha \in \mathbb{N}_{0}^{3}} \frac{|\alpha| !}{\alpha !} \zeta^{\alpha}(x) \\
\frac{\cdot\left(S \odot \zeta^{\alpha}(y)\right)}{}\end{array}$ \\
\hline $\begin{array}{c}\text { backward shift } \\
\text { operator }\end{array}$ & kernel eigenvector & kernel not eigenvector & $\begin{array}{l}\text { kernel common } \\
\text { eigenvector of the shifts }\end{array}$ \\
\hline rational functions & ring & group & ring \\
\hline
\end{tabular}


Remark 13.1. For the formulas for the adjoint operator, see e.g. [7, (3.9), p. 160] for the slice hyperholomorphic case and [24, Proposition 2.2. p. 34] for the Fueter series setting.

\section{Acknowledgements}

Daniel Alpay thanks the Foster G. and Mary McGaw Professorship in Mathematical Sciences, which supported this research. Kamal Diki acknowledges the support of the project INdAM Doctoral Programme in Mathematics and/or Applications Cofunded by Marie Sklodowska-Curie Actions, acronym: INdAM-DP-COFUND-2015, Grant No.: 713485.

Funding Open access funding provided by Politecnico di Milano within the CRUI-CARE Agreement.

Open Access. This article is licensed under a Creative Commons Attribution 4.0 International License, which permits use, sharing, adaptation, distribution and reproduction in any medium or format, as long as you give appropriate credit to the original author(s) and the source, provide a link to the Creative Commons licence, and indicate if changes were made. The images or other third party material in this article are included in the article's Creative Commons licence, unless indicated otherwise in a credit line to the material. If material is not included in the article's Creative Commons licence and your intended use is not permitted by statutory regulation or exceeds the permitted use, you will need to obtain permission directly from the copyright holder. To view a copy of this licence, visit http:// creativecommons.org/licenses/by/4.0/.

Publisher's Note Springer Nature remains neutral with regard to jurisdictional claims in published maps and institutional affiliations.

\section{References}

[1] Abu-Ghanem, Kh., Alpay, D., Colombo, F., Lewkowicz, I., Sabadini, I.: Herglotz functions of several quaternionic variables. J. Math. Anal. Appl. 466(1), 169-182 (2018)

[2] Abu-Ghanem, Kh., Alpay, D., Colombo, F., Sabadini, I.: Gleason's problem and Schur multipliers in the multivariable quaternionic setting. J. Math. Anal. Appl. 425(2), 1083-1096 (2015)

[3] Abul-Ez, M.A., Constales, D.: Basic sets of polynomials in Clifford analysis. Complex Var. Theory Appl. Int. J. 14(1-4), 177-185 (1990)

[4] Agler, J.: On the Representation of Certain Holomorphic Functions Defined on a Polydisk, volume 48 of Operator Theory: Advances and Applications, pp. 47-66. Birkhäuser Verlag, Basel (1990)

[5] Akhiezer, N.I.: The Classical Moment Problem. Moscow (1961). in Russian

[6] Alpay, D.: Algorithme de Schur, espaces à noyau reproduisant et théorie des systèmes. Panoramas et Synthèses [Panoramas and Syntheses], vol. 6. Société Mathématique de France, Paris (1998) 
[7] Alpay, D., Bolotnikov, V., Colombo, F., Sabadini, I.: Self-mappings of the quaternionic unit ball: multiplier properties, Schwarz-Pick inequality, and Nevanlinna-Pick interpolation problem. Indiana Univ. Math. J. 64, 151-180 (2015)

[8] Alpay, D., Colombo, F., Sabadini, I.: Slice Hyperholomorphic Schur Analysis, volume 256 of Operator Theory: Advances and Applications. Birkhäuser, Basel (2016)

[9] Alpay, D., Colombo, F., Sabadini, I.: Realizations of holomorphic and slice hyperholomorphic functions: the Krein space case. Indag. Math. (N.S.) 31(4), 607-628 (2020)

[10] Alpay, D., Correa-Romero, F.M., Luna-Elizarrarás, M.E., Shapiro, M.: Hyperholomorphic rational functions: the Clifford analysis case. Complex Var. Elliptic Equ. 52(1), 59-78 (2007)

[11] Alpay, D., Dijksma, A., Rovnyak, J., de Snoo, H.: Schur functions, operator colligations, and reproducing kernel Pontryagin spaces. In: Operator Theory: Advances and Applications, vol. 96. Birkhäuser Verlag, Basel (1997)

[12] Alpay, D., Diki, K., Sabadini, I.: Fock and Hardy spaces: Clifford Appell case. To appear in Mathematische Nachrichten (2021)

[13] Alpay, D., Diki, K., Sabadini, I.: On the global operator and Fueter mapping theorem for slice polyanalytic functions. To appear in Analysis and Applications. https://doi.org/10.1142/S0219530520500189

[14] Alpay, D., Diki, K., Sabadini, I.: On slice polyanalytic functions of a quaternionic variable. Res. Math. 74(1), 17 (2019)

[15] Alpay, D., Diki, K., Sabadini, I.: Correction to: On slice polyanalytic functions of a quaternionic variable. Res. Math. 76(2), 84 (2021)

[16] Alpay, D., Dym, H.: On applications of reproducing kernel spaces to the Schur algorithm and rational $J$-unitary factorization. In: Gohberg, I. (ed.) I. Schur Methods in Operator Theory and Signal Processing, Volume 18 of Operator Theory: Advances and Applications, pp. 89-159. Birkhäuser verlag, Basel (1986)

[17] Alpay, D., Gohberg, I.: Unitary rational matrix functions. In: Gohberg, I. (ed.) Topics in Interpolation Theory of Rational Matrix-Valued Functions. Operator Theory: Advances and Applications, vol. 33, pp. 175-222. Birkhäuser Verlag, Basel (1988)

[18] Alpay, D., Luna-Elizarrarás, M.E., Shapiro, M., Struppa, D.: Gleason's problem, rational functions and spaces of left-regular functions: the split-quaternion setting. Israel J. Math. 226(1), 319-349 (2018)

[19] Alpay, D., Paiva, I., Struppa, D.C.: A general setting for functions of Fueter variables: differentiability, rational functions, Fock module and related topics. Israel J. Math. 236, 207-246 (2020)

[20] Alpay, D., Schneider, B., Shapiro, M., Volok, D.: Fonctions rationnelles et théorie de la réalisation: le cas hyper-analytique. C. R. Math. 336, 975-980 (2003)

[21] Alpay, D., Shapiro, M.: Reproducing kernel quaternionic Pontryagin spaces. Integr. Equ. Oper. Theory 50, 431-476 (2004)

[22] Alpay, D., Shapiro, M., Volok, D.: Espaces de de Branges Rovnyak et fonctions de schur: le cas hyper-analytique. C. R. Math. 338, 437-442 (2004) 
[23] Alpay, D., Shapiro, M., Volok, D.: Rational hyperholomorphic functions in $R^{4}$. J. Funct. Anal. 221(1), 122-149 (2005)

[24] Alpay, D., Shapiro, M., Volok, D.: Reproducing kernel spaces of series of Fueter polynomials. In: Operator Theory in Krein Spaces and Nonlinear Eigenvalue Problems, Volume 162 of Operator Theory: Advances and Applications, pp. 19-45. Birkhäuser, Basel (2006)

[25] Alpay, D., Timoshenko, O., Volok, D.: Carathéodory functions in the Banach space setting. Linear Algebra Appl. 425, 700-713 (2007)

[26] Aronszajn, N.: Theory of reproducing kernels. Trans. Am. Math. Soc. 68, 337404 (1950)

[27] Ball, J., Bolotnikov, V.: Interpolation in the noncommutative Schur-Agler class. J. Oper. Theory 58(1), 83-126 (2007)

[28] Ball, J., Trent, T., Vinnikov, V.: Interpolation and commutant lifting for multipliers on reproducing kernel Hilbert spaces. In: Proceedings of Conference in Honor of the 60-th birthday of M.A. Kaashoek, Volume 122 of Operator Theory: Advances and Applications, pp. 89-138. Birkhauser (2001)

[29] Ball, J.A., Bolotnikov, V.: Canonical transfer-function realization for Schur multipliers on the Drury-Arveson space and models for commuting row contractions. Indiana Univ. Math. J. 61(2), 665-716 (2012)

[30] Ball, J.A., Kaliuzhnyi-Verbovetskyi, D.S.: Schur-Agler and Herglotz-Agler classes of functions: positive-kernel decompositions and transfer-function realizations. Adv. Math. 280, 121-187 (2015)

[31] Bart, H., Gohberg, I., Kaashoek, M.A.: Minimal factorization of matrix and operator functions. In: Operator Theory: Advances and Applications, vol. 1. Birkhäuser Verlag, Basel (1979)

[32] Brackx, F., Delanghe, R., Sommen, F.: Clifford Analysis, Volume 76. Pitman research notes (1982)

[33] Cação, I., Falcão, M.I., Malonek, H.: On generalized hypercomplex Laguerretype exponentials and applications. In: ICCSA 2011, Part III, LNCS 6784, pp. 271-286. Springer (2011)

[34] Cação, I., Falcão, M.I., Malonek, H.: Hypercomplex polynomials, Vietoris' rational numbers and a related integer numbers sequence. Complex Anal. Oper. Theory 11(5), 1059-1076 (2017)

[35] Colombo, F., Lávička, R., Sabadini, I., Souček, V.: The Radon transform between monogenic and generalized slice monogenic functions. Math. Ann. 363(34), 733-752 (2015)

[36] Colombo, F., Sabadini, I., Sommen, F.: The Fueter mapping theorem in integral form and the $\mathscr{F}$-functional calculus. Math. Methods Appl. Sci. 33(17), 20502066 (2010)

[37] Colombo, F., Sabadini, I., Sommen, F.: The inverse Fueter mapping theorem. Commun. Pure Appl. Anal. 10(4), 1165-1181 (2011)

[38] Colombo, F., Sabadini, I., Sommen, F., Struppa, D.C.: Analysis of Dirac Systems and Computational Algebra, Volume 39 of Progress in Mathematical Physics. Birkhäuser Boston Inc., Boston, MA (2004)

[39] Colombo, F., Sabadini, I., Struppa, D.C.: Noncommutative Functional Calculus, Volume 289 of Progress in Mathematics. Birkhäuser, Basel (2011). Theory and applications of slice hyperholomorphic functions 
[40] de Branges, L.: Some Hilbert spaces of analytic functions I. Trans. Am. Math. Soc. 106, 445-468 (1963)

[41] de Branges, L.: Espaces Hilbertiens de Fonctions Entières. Masson, Paris (1972)

[42] de Branges, L.: Complementation in Kreĭn spaces. Trans. Am. Math. Soc. 305, 277-291 (1988)

[43] de Branges, L., Rovnyak, J.: Canonical models in quantum scattering theory. In: Wilcox, C. (ed.) Perturbation Theory and Its Applications in Quantum Mechanics, pp. 295-392. Wiley, New York (1966)

[44] de Branges, L., Rovnyak, J.: Square Summable Power Series. Holt Rinehart and Winston, New York (1966)

[45] de Branges, L., Shulman, L.A.: Perturbation theory of unitary operators. J. Math. Anal. Appl. 23, 294-326 (1968)

[46] Delanghe, R.: On regular-analytic functions with values in a Clifford algebra. Math. Ann. 185, 91-111 (1970)

[47] Delanghe, R., Sommen, F., Souček, V.: Clifford Algebra and Spinor Valued Functions, Volume 53 of Mathematics and Its Applications. Kluwer Academic Publishers (1992)

[48] Delsarte, P., Genin, Y., Kamp, Y.: Schur parametrization of positive definite block-Toeplitz systems. SIAM J. Appl. Math. 36, 34-46 (1979)

[49] Diki, K., Krausshar, R.S., Sabadini, I.: On the Bargmann-Fock-Fueter and Bergman-Fueter integral transforms. J. Math. Phys. 60(8), 083506 (2019)

[50] Donoghue, W.F.: Monotone Matrix Functions and Analytic Continuation, Volume 207 of Die Grundlehren der Mathematischen Wissennschaften. Springer (1974)

[51] Dym, H.: J-contractive matrix functions, reproducing kernel Hilbert spaces and interpolation. Published for the Conference Board of the Mathematical Sciences, Washington, DC (1989)

[52] Dym, H., McKean, H.P.: Gaussian Processes, Function Theory and the Inverse Spectral Problem. Academic Press, Cambridge (1976)

[53] Falcao, M.I., Cruz, J.F., Malonek, H.R.: Remarks on the generation of monogenic functions. In: Proceedings of the 17th International Conference on te Application of Computer Science and Mathematical Architecture and Civil Engineering, Weimar, Germany, p. 22 (2007)

[54] Falcao, M.I., Malonek, H.R.: Generalized exponentials through Appell sets in $r^{n+1}$ and Bessel functions.In: Mathematics, T. Simos, G. Psihoyios and C. Tsitouras (eds.) Numerical Analysis and Applied. AIP Conference Proceedings 936. Melville, NY: American Institute of Physics (AIP) (2007)

[55] Fillmore, P.A., Williams, J.P.: On operator ranges. Adv. Math. 7, 254-281 (1971)

[56] Fliess, M.: Matrices de Hankel. J. Math. Pures Appl. 9(53), 197-222 (1974)

[57] Fricain, E., Mashreghi, J.: The Theory of $\mathcal{H}(b)$ Spaces. Vol. 1, Volume 20 of New Mathematical Monographs. Cambridge University Press, Cambridge (2016)

[58] Fricain, E., Mashreghi, J.: The Theory of $\mathcal{H}(b)$ spaces. Vol. 2, Volume 21 of New Mathematical Monographs. Cambridge University Press, Cambridge (2016)

[59] Fueter, R.: Über die analytische Darstellung der regulären Funktionen einer Quaternionenvariablen. Comment. Math. Helv. 8(1), 371-378 (1935) 
[60] Fuhrmann, P.A.: Linear Systems and Operators in Hilbert Space. McGraw-Hill International Book Company (1981)

[61] Gentili, G., Stoppato, C., Struppa, D.C.: Regular Functions of a Quaternionic Variable. Springer Monographs in Mathematics, Springer, Heidelberg (2013)

[62] Ghiloni, R., Perotti, A.: Slice regular functions on real alternative algebras. Adv. Math. 226(2), 1662-1691 (2011)

[63] Gürlebeck, K., Habetha, K., Sprößig, W.: Application of Holomorphic Functions in Two and Higher Dimensions. Springer, Berlin (2008)

[64] Guyker, J.: The de Branges-Rovnyak model. Proc. Am. Math. Soc. 111(1), 95-99 (1991)

[65] Guyker, J.: The de Branges-Rovnyak model with finite-dimensional coefficients. Trans. Am. Math. Soc. 347(4), 1383-1389 (1995)

[66] Hoffman, K.: Banach Spaces of Analytic Functions. Dover Publications Inc., New York (1988). Reprint of the 1962 original

[67] Kalman, R.E., Falb, P.L., Arbib, M.A.: Topics in Mathematical System Theory. McGraw-Hill Book Co., New York (1969)

[68] Kalyuzhnyı̆-Verbovetzkiŭ, D.: Carathéodory interpolation on the noncommutative polydisk. J. Funct. Anal. 229(2), 241-276 (2005)

[69] Kreŭn, M.G., Langer, H.: Über die verallgemeinerten Resolventen und die charakteristische Funktion eines isometrischen Operators im Raume $\Pi_{k}$. In Hilbert space operators and operator algebras (Proc. Int. Conf. Tihany, 1970), pp. 353-399. North-Holland: Colloquia Math. Soc, János Bolyai (1972)

[70] Kreı̆n, M.G., Langer, H.: Über die $Q$-Funktion eines $\pi$-hermiteschen Operators im Raume $\pi_{\kappa}$. Acta Sci. Math. (Szeged) 34, 191-230 (1973)

[71] Laville, G.: On Cauchy-Kovalewski extension. J. Funct. Anal. 101(1), 25-37 (1991)

[72] Leech, R.B.: On the characterization of $\mathcal{H}(B)$ spaces. Proc. Am. Math. Soc. 23, 518-520 (1969)

[73] Luger, A., Nedic, M.: A characterization of Herglotz-Nevanlinna functions in two variables via integral representations. Ark. Mat. 55(1), 199-216 (2017)

[74] Malonek, H.: A new hypercomplex structure of the Euclidean space $R^{m+1}$ and the concept of hypercomplex differentiability. Complex Var. Theory Appl. 14(1-4), 25-33 (1990)

[75] Meschkovski, H.: Hilbertsche Räume Mit Kernfunktion. Springer, Berlin (1962)

[76] Nikolski, N.K.: Operators, Functions, and Systems: An Easy Reading. Vol. 1, Volume 92 of Mathematical Surveys and Monographs. American Mathematical Society, Providence (2002). Hardy, Hankel, and Toeplitz, Translated from the French by Andreas Hartmann

[77] Reed, M., Simon, B.: Methods of Modern Mathematical Physics. I, second edn. Academic Press Inc. [Harcourt Brace Jovanovich Publishers], New York (1980). Functional analysis

[78] Rosenbrock, H.H.: State-Space and Multivariable Theory. Wiley, New York (1970)

[79] Saitoh, S.: Theory of Reproducing Kernels and Its Applications, vol. 189. Longman Scientific and Technical (1988)

[80] Schur, I.: Über die Potenzreihen, die im Innern des Einheitkreises beschränkten sind, I. J. Reine Angew. Math. 147, 205-232 (1917). English translation in: I. 
Schur methods in operator theory and signal processing. (Operator theory: Advances and Applications OT 18 (1986), Birkhäuser Verlag), Basel

[81] Schur, I.: Über die potenzreihen, die im Innern des Einheitkreises Beschrankt sind, II. J. Reine Angew. Math. 148, 122-145 (1918). English translation in: I. Schur methods in operator theory and signal processing. (Operator theory: Advances and Applications OT 18 (1986), Birkhäuser Verlag), Basel

[82] Sommen, F.: A product and an exponential function in hypercomplex function theory. Appl. Anal. 12(1), 13-26 (1981)

Daniel Alpay

Faculty of Mathematics, Physics, and Computation, Schmid College of Science and Technology

Chapman University

One University Drive

Orange CA92866

USA

e-mail: alpay@chapman.edu

Fabrizio Colombo, Kamal Diki and Irene Sabadini $(\bowtie)$

Politecnico di Milano

Dipartimento di Matematica

Via E. Bonardi, 9

20133 Milan

Italy

e-mail: irene.sabadini@polimi.it

Fabrizio Colombo

e-mail: fabrizio.colombo@polimi.it

\section{Kamal Diki}

e-mail: kamal.diki@polimi.it

Received: November 12, 2020.

Revised: May 22, 2021. 